\title{
Investigating the near-criticality of the Higgs boson
}

\author{
Dario Buttazzo, ${ }^{a, b}$ Giuseppe Degrassi, ${ }^{c}$ Pier Paolo Giardino, ${ }^{a, d}$ Gian F. Giudice, ${ }^{a}$ \\ Filippo Sala, ${ }^{b, e}$ Alberto Salvio ${ }^{b, f}$ and Alessandro Strumia ${ }^{d}$ \\ ${ }^{a}$ CERN, Theory Division, \\ CH-1211 Geneva 23, Switzerland \\ ${ }^{b}$ Scuola Normale Superiore and INFN, sezione di Pisa, \\ Piazza dei Cavalieri 7, Pisa, Italy \\ ${ }^{c}$ Dipartimento di Matematica e Fisica, Università di Roma Tre and \\ INFN, sezione di Roma Tre, \\ Via della Vasca Navale 84, Rome, Italy \\ ${ }^{d}$ Dipartimento di Fisica, Università di Pisa and INFN, sezione di Pisa, \\ Largo Bruno Pontecorvo 3, Pisa, Italy \\ e Theoretical Physics Group, Lawrence Berkeley National Laboratory, \\ 1 Cyclotron Rd., Berkeley, CA, U.S.A. \\ ${ }^{f}$ Departamento de Fúsica Teórica, Universidad Autónoma de Madrid and \\ Instituto de Física Teórica IFT-UAM/CSIC, \\ C/ Nicolás Cabrera 13-15, Madrid, Spain \\ E-mail: dario.buttazzo@sns.it, degrassi@fis.uniroma3.it, \\ pierpaolo.giardino@pi.infn.it, Gian.Giudice@cern.ch, \\ filippo.sala@sns.it, alberto.salvio@uam.es, astrumia@cern.ch
}

ABSTRACT: We extract from data the parameters of the Higgs potential, the top Yukawa coupling and the electroweak gauge couplings with full 2-loop NNLO precision, and we extrapolate the SM parameters up to large energies with full 3-loop NNLO RGE precision. Then we study the phase diagram of the Standard Model in terms of high-energy parameters, finding that the measured Higgs mass roughly corresponds to the minimum values of the Higgs quartic and top Yukawa and the maximum value of the gauge couplings allowed by vacuum metastability. We discuss various theoretical interpretations of the near-criticality of the Higgs mass.

KeYwords: Higgs Physics, Standard Model, Renormalization Group

ARXIV EPRINT: 1307.3536 


\section{Contents}

1 Introduction 1

2 Computing the $\overline{\mathrm{MS}}$ parameters with two-loop accuracy 2

2.1 Two-loop correction to the Higgs quartic coupling 7

2.2 Two-loop correction to the Higgs mass term 9

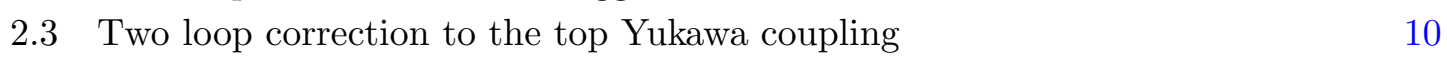

$\begin{array}{ll}2.4 & \text { Two-loop correction to weak and hypercharge gauge couplings } \\ \end{array}$

3 SM couplings at the electroweak scale $\quad 12$

$\begin{array}{ll}3.1 \text { The Higgs quartic coupling } & 13\end{array}$

$\begin{array}{lll}3.2 & \text { The Higgs mass term } & 13\end{array}$

$\begin{array}{ll}3.3 \text { The top Yukawa coupling } & 13\end{array}$

$\begin{array}{ll}3.4 \text { The weak gauge couplings } & 13\end{array}$

$\begin{array}{ll}3.5 & \text { The strong gauge coupling } \\ \end{array}$

4 Extrapolation of the SM up to the Planck scale $\quad 14$

$\begin{array}{lll}\text { 4.1 SM couplings at the Planck scale } & 14\end{array}$

$\begin{array}{ll}4.2 \text { Derivation of the stability bound } & 17\end{array}$

4.3 The SM phase diagram in terms of Higgs and top masses 17

$\begin{array}{ll}\text { 4.4 The SM phase diagram in terms of Planck-scale couplings } & 19\end{array}$

5 Interpretations of the high-energy SM couplings $\quad 21$

$\begin{array}{lll}5.1 & \text { Matching conditions } & 21\end{array}$

5.2 Criticality as an attractor 21

5.3 Double criticality of Higgs and top couplings 23

$\begin{array}{lll}5.4 \text { Statistics } & 23\end{array}$

6 More on SM phase diagrams $\quad \mathbf{2 5}$

6.1 The SM phase diagram in terms of gauge couplings 25

6.2 The SM phase diagram in terms of Higgs potential parameters 27

$\begin{array}{ll}6.3 & \text { Lifetime of the SM vacuum } \\ \end{array}$

$\begin{array}{lll}7 & \text { Summary and conclusions } & 30\end{array}$

A Weak scale thresholds at one loop $\quad 33$

A.1 The quartic Higgs coupling 34

A.2 The Higgs mass term 34

A.3 The top Yukawa coupling 34

$\begin{array}{lll}\text { A.4 The weak gauge couplings } & 35\end{array}$ 
B SM RGE equations up to three loops

B.1 Gauge couplings 36

B.2 Higgs quartic coupling 36

B.3 Higgs mass term 37

$\begin{array}{lll}\text { B.4 Yukawa couplings } & 37\end{array}$

$\begin{array}{lll}\text { C Effective potential at two loops } & 38\end{array}$

\section{Introduction}

The discovery of the Higgs boson [1,2] was expected to be the herald of new physics soon to be found at the TeV scale. So far, however, no signal of new physics nor any clear deviation from the SM Higgs properties have been detected at the LHC. Moreover, the Higgs mass has not provided unambiguous indications for new physics. The measured value $M_{h}=$ $125.66 \pm 0.34 \mathrm{GeV}[3-7]$ is a bit high for supersymmetry and a bit low for composite models, making theoretical interpretations rather uncomfortable. Neither option is unequivocally favoured, although neither option is excluded. On the other hand, $M_{h}=125.66 \pm 0.34 \mathrm{GeV}$ lies well within the parameter window in which the SM can be extrapolated all the way up to the Planck mass $M_{\mathrm{Pl}}$, with no problem of consistency other than remaining in the dark about naturalness. Remarkably, in the context of the SM the measured value of $M_{h}$ is special because it corresponds to a near-critical situation in which the Higgs vacuum does not reside in the configuration of minimal energy, but in a metastable state close to a phase transition [8] (for earlier considerations see [9-31]; for related studies see [32-57]).

We believe that near-criticality of the SM vacuum is the most important message we have learnt so far from experimental data on the Higgs boson. Near-criticality gives us a unique opportunity to obtain information about physics taking place at energy scales well beyond the reach of any collider experiment. Its consequences are so intriguing and potentially so revolutionary that they deserve accurate calculations and dedicated studies. In this paper we continue our programme of investigating the status and implications of near-criticality. We make advancements on both sides: on the computational side, we improve the calculation of the large-field extrapolation of the Higgs potential and of the critical value of $M_{h}$ for absolute stability; on the interpretation side, we explore the significance of near-criticality in terms of high-energy SM parameters.

The main new calculations presented in this paper are the results for the $\overline{\mathrm{MS}}$ quartic Higgs coupling $\lambda(\bar{\mu})$, for the top Yukawa coupling $y_{t}(\bar{\mu})$, for the electroweak gauge couplings at NNLO precision (two loops) in terms of physical observables: the pole masses of the Higgs $\left(M_{h}\right)$, of the top $\left(M_{t}\right)$, of the $Z\left(M_{Z}\right)$, of the $W\left(M_{W}\right)$, the $\overline{\mathrm{MS}}$ strong coupling $\alpha_{3}\left(M_{Z}\right)$, and the Fermi constant $G_{\mu}$. We improve on the study in ref. [8] where 2-loop threshold corrections to $\lambda(\bar{\mu})$ had been computed in the limit of vanishing weak gauge couplings, and 2-loop electroweak threshold corrections to $y_{t}(\bar{\mu})$ had been neglected. As 
a byproduct of our two-loop calculation of $\lambda(\bar{\mu})$ we also obtain the $\overline{\mathrm{MS}}$ quadratic Higgs coupling $m^{2}(\bar{\mu})$ at the NNLO level.

Recently, many authors have contributed towards the completion of the calculation of the renormalisation-group ( $\mathrm{RG}$ ) evolution ( $\beta$-functions and thresholds) of the sizeable SM couplings at NNLO precision. We summarise the present status of these calculations in table 1. Our new calculation of threshold corrections, together with the results collected in table 1, allows us to refine the determination of the critical value of $M_{h}$ that ensures absolute vacuum stability within the Standard Model (SM) up to the Planck scale. Furthermore, our precision extrapolation of the SM to high energy scales is relevant for testing any new physics scenario able of making predictions, such as unification of gauge couplings constants, or high-scale supersymmetric models that restrict or predict the quartic Higgs coupling.

The paper is organised as follows. In section 2 we outline the general strategy for the two-loop computations and describe our new results. In section 3 we present numerical results for the $\overline{\mathrm{MS}}$ couplings at the weak scale. The implications of these results for Planck scale physics are discussed in sections 4-6. The results are summarised in the conclusions. We complemented the paper with several appendices where we collect all the known results on the RG equations and the threshold corrections that we used in our computation.

\section{Computing the $\overline{\mathrm{MS}}$ parameters with two-loop accuracy}

In this section we first outline the general strategy followed to determine the $\overline{\mathrm{MS}}$ parameters in terms of physical observables at the two-loop level. Then, in sects. 2.1 and 2.2, we will discuss the results for the quartic and quadratic Higgs couplings, respectively, while section 2.3 is dedicated to the calculation of two-loop threshold corrections to the top Yukawa coupling.

First of all, all $\overline{\mathrm{MS}}$ parameters have gauge-invariant renormalisation group equations [88, 89] and are gauge invariant, as we now prove. ${ }^{1}$ Let us consider a generic $\overline{\mathrm{MS}}$ coupling $\theta$ measuring the strength of a gauge invariant term in the Lagrangian and a generic gauge fixing parametrized by $\xi$ (for example the $R_{\xi}$ gauges). Let us first recall the definition of $\theta$ in terms of the bare coupling $\theta_{0}$,

$$
\bar{\mu}^{d-4} \theta_{0}=\sum_{k=0}^{\infty} \frac{c_{k}(\theta, \xi)}{(d-4)^{k}},
$$

where the $c_{k}$ are defined to be the residues at the divergence $d=4$. The important point is that $c_{0}=\theta$, with no dependence on $\xi$. Since $\theta_{0}$ is gauge independent, we have

$$
0=\bar{\mu}^{d-4} \frac{d \theta_{0}}{d \xi}=\frac{d \theta}{d \xi}+\sum_{k=1}^{\infty} \frac{1}{(d-4)^{k}} \frac{d c_{k}(\theta, \xi)}{d \xi} .
$$

Since this equation is valid for any $d$, and $\theta$ has no poles at $d=4$ by definition, we obtain $d \theta / d \xi=0$, that is $\theta$ is gauge invariant (as well as all the residues $\left.c_{k}\right)^{2}{ }^{2}$

\footnotetext{
${ }^{1}$ Gauge invariance of fermion pole masses has been proved in refs. [90-92] and here we generalise their proof.

${ }^{2}$ Notice that this proof does not apply to the Higgs vev $v$, because it is not the coefficient of a gaugeinvariant term in the Lagrangian.
} 
Renormalisation Group Equations

\begin{tabular}{|c|cccc|}
\hline \multirow{4}{*}{$g_{3}$} & LO & NLO & NNLO & NNNLO \\
& 1 loop & 2 loop & 3 loop & 4 loop \\
& full $[58,59]$ & $\mathcal{O}\left(\alpha_{3}^{2}\right)[60,61]$ & $\mathcal{O}\left(\alpha_{3}^{3}\right)[62,63]$ & $\mathcal{O}\left(\alpha_{3}^{4}\right)[64,65]$ \\
& & $\mathcal{O}\left(\alpha_{3} \alpha_{1,2}\right)[66]$ & $\mathcal{O}\left(\alpha_{3}^{2} \alpha_{t}\right)[67]$ & \\
$g_{1,2}$ & full $[58,59]$ & full $[68]$ & full $[69,70]$ & \\
$y_{t}$ & full $[71]$ & $\mathcal{O}\left(\alpha_{t}^{2}, \alpha_{3} \alpha_{t}\right)[72]$ & full $[69,70]$ & - \\
& & full $[75]$ & full $[73,74]$ & - \\
$\lambda, m^{2}$ & full $[71]$ & full $[76,77]$ & full $[78,79]$ & - \\
\hline
\end{tabular}

Threshold corrections at the weak scale

\begin{tabular}{|c|cccc|}
\hline & LO & NLO & NNLO & NNNLO \\
& 0 loop & 1 loop & 2 loop & 3 loop \\
\hline$g_{2}$ & $2 M_{W} / V$ & full $[80,81]$ & full [This work] & - \\
$g_{Y}$ & $2 \sqrt{M_{Z}^{2}-M_{W}^{2}} / V$ & full $[80,81]$ & full [This work] & - \\
$y_{t}$ & $\sqrt{2} M_{t} / V$ & $\mathcal{O}\left(\alpha_{3}\right)[82]$ & $\mathcal{O}\left(\alpha_{3}^{2}, \alpha_{3} \alpha_{1,2}\right)[38]$ & $\mathcal{O}\left(\alpha_{3}^{3}\right)[83-85]$ \\
& & $\mathcal{O}(\alpha)[86]$ & full [This work] & \\
$\lambda$ & $M_{h}^{2} / 2 V^{2}$ & full $[87]$ & for $g_{1,2}=0[8]$ & - \\
& & & full [This work] & \\
$m^{2}$ & $M_{h}^{2}$ & full [87] & full [This work] & - \\
\hline
\end{tabular}

Table 1. Present status of higher-order computations included in our code. With the present paper the calculation of the SM parameters at NNLO precision is complete. Here we have defined $V \equiv\left(\sqrt{2} G_{\mu}\right)^{-1 / 2}$ and $g_{1}=\sqrt{5 / 3} g_{Y}$.

To determine the $\overline{\mathrm{MS}}$ parameters in terms of physical observables two strategies can be envisaged.

i) Perform an $\overline{\mathrm{MS}}$ renormalisation to obtain directly the $\overline{\mathrm{MS}}$ quantity of interest in terms of $\overline{\mathrm{MS}}$ parameters. Then express the $\overline{\mathrm{MS}}$ parameters in terms of the physical ones via appropriately derived two-loop relations.

ii) Use a renormalisation scheme in which the renormalised parameters are directly expressed in terms of physical observables (we call this scheme generically on-shell (OS) and label quantities in this scheme with an OS). Then relate the parameters as expressed in the OS scheme to their $\overline{\mathrm{MS}}$ counterparts we are looking for. 


\begin{tabular}{|c|c|c|}
\hline$M_{W}=80.384 \pm 0.014 \mathrm{GeV}$ & Pole mass of the $W$ boson & {$[93,94]$} \\
\hline$M_{Z}=91.1876 \pm 0.0021 \mathrm{GeV}$ & Pole mass of the $Z$ boson & [95] \\
\hline$M_{h}=125.66 \pm 0.34 \mathrm{GeV}$ & he higgs & {$[3-7]$} \\
\hline$M_{t}=173.10 \pm 0.59 \pm 0.3 \mathrm{GeV}$ & Pole mass of the top quark & {$[96-99]$} \\
\hline$\left(2 G_{\mu}\right)^{-1 / 2}=246.21971 \pm 0.00006 \mathrm{GeV}$ & Fermi constant for $\mu$ decay & [100] \\
\hline$\alpha_{3}\left(M_{Z}\right)=0.1184 \pm 0.0007$ & $\overline{\mathrm{MS}}$ gauge $\mathrm{SU}(3)_{c}$ coupling (5 flavours) & [101] \\
\hline
\end{tabular}

Table 2. Input values of the SM observables used to fix the SM fundamental parameters $\lambda, m, y_{t}, g_{2}, g_{Y}$. The pole top mass, $M_{t}$, is a naive average of TeVatron, CMS, ATLAS measurements, all extracted from difficult MonteCarlo modellings of top decay and production in hadronic collisions. Furthermore, $M_{t}$ is also affected by a non-perturbative theoretical uncertainty of order $\Lambda_{\mathrm{QCD}}$, that we quantify as $\pm 0.3 \mathrm{GeV}$. Throughout the paper we give explicitly the dependence of all physical quantities on $M_{t}$, and thus the impact of larger theoretical uncertainties on the top mass is always manifest in our results.

This last step can be easily done using the relation

$$
\theta_{0}=\theta_{\mathrm{OS}}-\delta \theta_{\mathrm{OS}}=\theta(\bar{\mu})-\delta \theta_{\overline{\mathrm{MS}}}
$$

or

$$
\theta(\bar{\mu})=\theta_{\mathrm{OS}}-\delta \theta_{\mathrm{OS}}+\delta \theta_{\overline{\mathrm{MS}}},
$$

where $\theta_{0}$ is the bare parameter, $\theta(\bar{\mu})\left(\theta_{\mathrm{OS}}\right)$ is the renormalised $\overline{\mathrm{MS}}(\mathrm{OS})$ version and $\delta \theta_{\overline{\mathrm{MS}}}\left(\delta \theta_{\mathrm{OS}}\right)$ the corresponding counterterm. By definition $\delta \theta_{\overline{\mathrm{MS}}}$ subtracts only the terms proportional to powers of $1 / \epsilon$ and $\gamma-\ln (4 \pi)$ in dimensional regularisation, with $d=4-2 \epsilon$ being the space-time dimension. Concerning the structure of the $1 / \epsilon$ poles in the OS and $\overline{\mathrm{MS}}$ counterterms, one notices that it should be identical once the poles in the OS counterterms are expressed in terms of $\overline{\mathrm{MS}}$ quantities. Then, after this operation is performed, the desired $\theta(\bar{\mu})$ is obtained from

$$
\theta(\bar{\mu})=\theta_{\mathrm{OS}}-\left.\delta \theta_{\mathrm{OS}}\right|_{\mathrm{fin}}+\Delta_{\theta},
$$

where the subscript 'fin' denotes the finite part of the quantity involved and $\Delta$ is the two-loop finite contribution that is obtained when the OS parameters entering the $1 / \epsilon$ pole in the OS counterterm are expressed in terms of $\overline{\mathrm{MS}}$ quantities, the finite contribution coming from the $\mathcal{O}(\epsilon)$ part of the shifts.

In the following we adopt strategy ii).

The quantities of interests are $\theta=\left(m^{2}, \lambda, v, y_{t}, g_{2}, g_{1}\right)$, i.e. the quadratic and quartic couplings in the Higgs potential, the vacuum expectation value (vev), the top Yukawa coupling, the $\mathrm{SU}(2)_{L}$ and $\mathrm{U}(1)_{Y}$ gauge couplings $g_{2}$ and $g_{Y}$ (with $g_{1}=\sqrt{5 / 3} g_{Y}$ being the hypercharge coupling rewritten in $\mathrm{SU}(5)$ normalisation), and are directly determined in terms of the pole masses of the Higgs $\left(M_{h}\right)$, of the top $\left(M_{t}\right)$, of the $Z\left(M_{Z}\right)$, of the $W$ $\left(M_{W}\right)$, the Fermi constant $G_{\mu}$ and the $\overline{\mathrm{MS}}$ strong coupling $\alpha_{3}\left(M_{Z}\right)$. Their input values are listed in table 2. Then, using eq. (2.5), the $\overline{\mathrm{MS}}$ quantities are obtained. We notice that the 
weak-scale values for the $\overline{\mathrm{MS}}$ gauge couplings at the scale $\bar{\mu}$ are given in terms of $G_{\mu}, M_{W}$ and $M_{Z}$ and not in terms of the fine structure constant and the weak mixing angle at the $M_{Z}$ scale as usually done.

In order to fix the notation we write the classical Higgs potential as (the subscript 0 indicates a bare quantity)

$$
V_{0}=-\frac{m_{0}^{2}}{2}\left|H_{0}\right|^{2}+\lambda_{0}\left|H_{0}\right|^{4} .
$$

The classical Higgs doublet $H_{0}$ is defined by

$$
H_{0}=\left(\begin{array}{c}
\chi \\
\left(v_{0}+h+i \eta\right) / \sqrt{2}
\end{array}\right)
$$

in terms of the physical Higgs field $h$, and of the neutral and charged would-be Goldstone bosons $\eta$ and $\chi$. The renormalisation of the Higgs potential, eq. (2.6), was discussed at the one-loop level in [87] and extended at the two-loop level in [8]. We refer to these papers for details. We recall that in ref. [8] the renormalised vacuum is identified with the minimum of the radiatively corrected potential ${ }^{3}$ and it is defined through $G_{\mu}$. Writing the relation between the Fermi constant and the bare vacuum as

$$
\frac{G_{\mu}}{\sqrt{2}}=\frac{1}{2 v_{0}^{2}}\left(1+\Delta r_{0}\right),
$$

one gets

$$
v_{\mathrm{OS}}^{2}=\frac{1}{\sqrt{2} G_{\mu}}, \quad \delta v_{\mathrm{OS}}^{2}=-\frac{\Delta r_{0}}{\sqrt{2} G_{\mu}} .
$$

The quadratic and quartic couplings in the Higgs potential are defined through $M_{h}$ via

$$
m_{\mathrm{OS}}^{2}=2 \lambda_{\mathrm{OS}} v_{\mathrm{OS}}^{2}, \quad M_{h}^{2}=2 \lambda_{\mathrm{OS}} v_{\mathrm{OS}}^{2},
$$

or

$$
\lambda_{\mathrm{OS}}=\frac{G_{\mu}}{\sqrt{2}} M_{h}^{2}, \quad m_{\mathrm{OS}}^{2}=M_{h}^{2} .
$$

Writing the counterterm for the quartic Higgs coupling as

$$
\delta \lambda_{\mathrm{OS}}=\delta^{(1)} \lambda_{\mathrm{OS}}+\delta^{(2)} \lambda_{\mathrm{OS}},
$$

where the superscript indicates the loop order, one finds

$$
\begin{aligned}
\delta^{(1)} \lambda_{\mathrm{OS}}= & \frac{G_{\mu}}{\sqrt{2}} M_{h}^{2}\left\{\Delta r_{0}^{(1)}+\frac{1}{M_{h}^{2}}\left[\frac{T^{(1)}}{v_{\mathrm{OS}}}+\delta^{(1)} M_{h}^{2}\right]\right\}, \\
\delta^{(2)} \lambda_{\mathrm{OS}}= & \frac{G_{\mu}}{\sqrt{2}} M_{h}^{2}\left\{\Delta r_{0}^{(2)}+\frac{1}{M_{h}^{2}}\left[\frac{T^{(2)}}{v_{\mathrm{OS}}}+\delta^{(2)} M_{h}^{2}\right]+\right. \\
& \left.-\Delta r_{0}^{(1)}\left(\Delta r_{0}^{(1)}+\frac{1}{M_{h}^{2}}\left[\frac{3 T^{(1)}}{2 v_{\mathrm{OS}}}+\delta^{(1)} M_{h}^{2}\right]\right)\right\} .
\end{aligned}
$$

\footnotetext{
${ }^{3}$ This condition is enforced choosing the tadpole counterterm to cancel completely the tadpole graphs.
} 
In eqs. (2.13)-(2.14) $i T$ represents the sum of the tadpole diagrams with external leg extracted, and $\delta M_{a}^{2}$ labels the mass counterterm for the particle $a$.

Similarly, one finds for the counterterm of the quadratic Higgs coupling in the potential

$$
\begin{aligned}
& \delta^{(1)} m_{\mathrm{OS}}^{2}=3 \frac{T^{(1)}}{v_{\mathrm{OS}}}+\delta^{(1)} M_{h}^{2}, \\
& \delta^{(2)} m_{\mathrm{OS}}^{2}=3 \frac{T^{(2)}}{v_{\mathrm{OS}}}+\delta^{(2)} M_{h}^{2}-\frac{3 T^{(1)}}{2 v_{\mathrm{OS}}} \Delta r_{0}^{(1)} .
\end{aligned}
$$

The top Yukawa and gauge couplings are fixed using $M_{t}, M_{W}$ and $M_{Z}$ via

$$
M_{t}=\frac{y_{t_{\mathrm{OS}}}}{\sqrt{2}} v_{\mathrm{OS}}, \quad M_{W}^{2}=\frac{g_{2_{\mathrm{OS}}}^{2}}{4} v_{\mathrm{OS}}^{2}, \quad M_{Z}^{2}=\frac{g_{2_{\mathrm{OS}}}^{2}+g_{Y_{\mathrm{OS}}}^{2}}{4} v_{\mathrm{OS}}^{2},
$$

or

$$
y_{t_{\mathrm{OS}}}=2\left(\frac{G_{\mu}}{\sqrt{2}} M_{t}^{2}\right)^{1 / 2}, \quad g_{2_{\mathrm{OS}}}=2\left(\sqrt{2} G_{\mu}\right)^{1 / 2} M_{W}, \quad g_{Y_{\mathrm{OS}}}=2\left(\sqrt{2} G_{\mu}\right)^{1 / 2} \sqrt{M_{Z}^{2}-M_{W}^{2}} .
$$

The corresponding counterterms are found to be

$$
\begin{aligned}
& \delta^{(1)} y_{t_{\mathrm{OS}}}=2\left(\frac{G_{\mu}}{\sqrt{2}} M_{t}^{2}\right)^{1 / 2}\left(\frac{\delta^{(1)} M_{t}}{M_{t}}+\frac{\Delta r_{0}^{(1)}}{2}\right) \\
& \delta^{(2)} y_{t_{\mathrm{OS}}}=2\left(\frac{G_{\mu}}{\sqrt{2}} M_{t}^{2}\right)^{1 / 2}\left(\frac{\delta^{(2)} M_{t}}{M_{t}}+\frac{\Delta r_{0}^{(2)}}{2}-\frac{\Delta r_{0}^{(1)}}{2}\left[\frac{\delta^{(1)} M_{t}}{M_{t}}+\frac{3 \Delta r_{0}^{(1)}}{4}\right]\right),
\end{aligned}
$$

for the top Yukawa coupling, and

$$
\begin{aligned}
\delta^{(1)} g_{2_{\mathrm{OS}}}= & \left(\sqrt{2} G_{\mu}\right)^{1 / 2} M_{W}\left(\frac{\delta^{(1)} M_{W}^{2}}{M_{W}^{2}}+\Delta r_{0}^{(1)}\right) \\
\delta^{(2)} g_{2_{\mathrm{OS}}}= & \left(\sqrt{2} G_{\mu}\right)^{1 / 2} M_{W}\left(\frac{\delta^{(2)} M_{W}^{2}}{M_{W}^{2}}+\Delta r_{0}^{(2)}+\right. \\
& \left.-\frac{\Delta r_{0}^{(1)}}{2}\left[\frac{\delta^{(1)} M_{W}^{2}}{M_{W}^{2}}+\frac{3 \Delta r_{0}^{(1)}}{2}\right]+\frac{1}{4}\left(\frac{\delta^{(1)} M_{W}^{2}}{M_{W}^{2}}\right)^{2}\right),
\end{aligned}
$$

for the $\mathrm{SU}(2)_{L}$ gauge coupling, and

$$
\begin{aligned}
\delta^{(1)} g_{Y_{\mathrm{OS}}}= & \left(\sqrt{2} G_{\mu}\right)^{1 / 2} \sqrt{M_{Z}^{2}-M_{W}^{2}}\left(\frac{\delta^{(1)} M_{Z}^{2}-\delta^{(1)} M_{W}^{2}}{M_{Z}^{2}-M_{W}^{2}}+\Delta r_{0}^{(1)}\right), \\
\delta^{(2)} g_{Y_{\mathrm{OS}}}= & \left(\sqrt{2} G_{\mu}\right)^{1 / 2} \sqrt{M_{Z}^{2}-M_{W}^{2}}\left(\frac{\delta^{(2)} M_{Z}^{2}-\delta^{(2)} M_{W}^{2}}{M_{Z}^{2}-M_{W}^{2}}+\Delta r_{0}^{(2)}+\right. \\
& \left.-\frac{\Delta r_{0}^{(1)}}{2}\left[\frac{\delta^{(1)} M_{Z}^{2}-\delta^{(1)} M_{W}^{2}}{M_{Z}^{2}-M_{W}^{2}}+\frac{3 \Delta r_{0}^{(1)}}{2}\right]+\frac{1}{4}\left(\frac{\delta^{(1)} M_{Z}^{2}-\delta^{(1)} M_{W}^{2}}{M_{Z}^{2}-M_{W}^{2}}\right)\right) .
\end{aligned}
$$

for the hypercharge gauge coupling. 


\subsection{Two-loop correction to the Higgs quartic coupling}

The $\overline{\mathrm{MS}}$ Higgs quartic coupling is given by

$$
\lambda(\bar{\mu})=\frac{G_{\mu}}{\sqrt{2}} M_{h}^{2}+\lambda^{(1)}(\bar{\mu})+\lambda^{(2)}(\bar{\mu})
$$

with

$$
\begin{aligned}
& \lambda^{(1)}(\bar{\mu})=-\left.\delta^{(1)} \lambda_{\mathrm{OS}}\right|_{\text {fin }}, \\
& \lambda^{(2)}(\bar{\mu})=-\left.\delta^{(2)} \lambda_{\mathrm{OS}}\right|_{\text {fin }}+\Delta_{\lambda} .
\end{aligned}
$$

The one-loop contribution in eq. $(2.25), \lambda^{(1)}$, is given by the finite part of eq. (2.13). Concerning the two-loop part, $\lambda^{(2)}(\bar{\mu})$, the QCD corrections were presented in refs. [8, 38], and the two-loop electroweak $(\mathrm{EW})$ part, $\lambda_{\mathrm{EW}}^{(2)}(\bar{\mu})$, was computed in ref. [8] in the so-called gauge-less limit of the SM, in which the electroweak gauge interactions are switched off. The main advantage of this limit results in a simplified evaluation of $\Delta r_{0}^{(2)}$. The computation of the two-loop EW part in the full SM requires instead the complete evaluation of this quantity and we outline here the derivation of $\lambda_{\mathrm{EW}}^{(2)}(\bar{\mu})$ starting from the term $\Delta r_{0}^{(2)}$ in $\delta^{(2)} \lambda_{\mathrm{OS}}$.

We recall that the Fermi constant is defined in terms of the muon lifetime $\tau_{\mu}$ as computed in the 4 -fermion $V-A$ Fermi theory supplemented by QED interactions. We extract $G_{\mu}$ from $\tau_{\mu}$ via

$$
\frac{1}{\tau_{\mu}}=\frac{G_{\mu}^{2} m_{\mu}^{5}}{192 \pi^{3}} F\left(\frac{m_{e}^{2}}{m_{\mu}^{2}}\right)(1+\Delta q)\left(1+\frac{3 m_{\mu}^{2}}{5 M_{W}^{2}}\right),
$$

where $F(\rho)=1-8 \rho+8 \rho^{3}-\rho^{4}-12 \rho^{2} \ln \rho=0.9981295$ (for $\rho=m_{e}^{2} / m_{\mu}^{2}$ ) is the phase space factor and $\Delta q=\Delta q^{(1)}+\Delta q^{(2)}=(-4.234+0.036) \times 10^{-3}$ are the QED corrections computed at one [102] and two loops [103]. From the measurement $\tau_{\mu}=(2196980.3 \pm 2.2) \mathrm{ps}$ [100] we find $G_{\mu}=1.1663781(6) 10^{-5} / \mathrm{GeV}^{2}$. This is $1 \sigma$ lower than the value quoted in [100] because we do not follow the convention of including in the definition of $G_{\mu}$ itself the last term of (2.27), which is the contribution from dimension-8 SM operators.

The computation of $\Delta r_{0}$ requires the subtraction of the QED corrections by matching the result in the SM with that in the Fermi theory. However, it is well known that the Fermi theory is renormalisable to all order in the electromagnetic interaction but to lowest order in $G_{\mu}$ due to a Ward identity that becomes manifest if the 4 -fermion interaction is rewritten via a Fierz transformation in the "charge retention order". As a consequence, in the limit of neglecting the fermion masses, $\Delta r_{0}$ as computed in the Fermi theory vanishes and we are just left with the calculation in the SM. ${ }^{4}$

Starting from eq. (2.8) we write $\Delta r_{0}$ as a sum of different terms:

$$
\Delta r_{0}=V_{W}-\frac{A_{W W}}{M_{W 0}^{2}}+2 v_{0}^{2} \mathcal{B}_{W}+\mathcal{E}+\mathcal{M},
$$

where $M_{W 0}$ is the bare $\mathrm{W}$ mass; $A_{W W}$ is the $W$ self-energy at zero momentum, $A_{W W}=$ $A_{W W}(0) ; V_{W}$ is the vertex contribution; $\mathcal{B}_{W}$ is the box contribution; $\mathcal{E}$ is the term due

\footnotetext{
${ }^{4}$ We explicitly verified that $\Delta r_{0}$ vanishes when computed in the Fermi theory.
} 
to the renormalisation of the external legs; $\mathcal{M}$ is the mixed contribution due to product of different objects among $V_{W}, A_{W W}, \mathcal{B}_{W}$ and $\mathcal{E}$ (see below for an explicit expression at two-loops). All quantities in eq. (2.28) are computed at zero external momenta. We point out that in the right-hand side of eq. (2.28) no tadpole contribution is included because of our choice of identifying the renormalised vacuum with the minimum of the radiatively corrected potential. As a consequence $\Delta r_{0}$ is a gauge-dependent quantity.

From eq. (2.28) the one-loop term is given by:

$$
\Delta r_{0}^{(1)}=V_{W}^{(1)}-\frac{A_{W W}^{(1)}}{M_{W}^{2}}+\frac{\sqrt{2}}{G_{\mu}} \mathcal{B}_{W}^{(1)}+\mathcal{E}^{(1)},
$$

where we have used that $\mathcal{M}^{(1)}=0$, while at two-loops

$$
\begin{aligned}
\Delta r_{0}^{(2)}= & V_{W}^{(2)}-\frac{A_{W W}^{(2)}}{M_{W}^{2}}+\sqrt{2} \frac{\mathcal{B}_{W}^{(2)}}{G_{\mu}}+\mathcal{E}^{(2)}+\mathcal{M}^{(2)}+ \\
& -\delta^{(1)} M_{W}^{2} \frac{A_{W W}^{(1)}}{M_{W}^{4}}+\frac{\sqrt{2}}{G_{\mu}} \mathcal{B}_{W}^{(1)}\left(V_{W}^{(1)}-\frac{A_{W W}^{(1)}}{M_{W}^{2}}+\sqrt{2} \frac{\mathcal{B}_{W}^{(1)}}{G_{\mu}}+\mathcal{E}^{(1)}\right)
\end{aligned}
$$

Here

$$
\delta^{(1)} M_{W}^{2}=\operatorname{Re} \Pi_{W W}\left(M_{W}^{2}\right)
$$

with $\Pi_{W W}\left(M_{W}^{2}\right)$ the $W$ boson self-energy evaluated at external momentum equal to $M_{W}$, and

$$
\mathcal{M}^{(2)}=\frac{\sqrt{2}}{G_{\mu}} \mathcal{E}^{(1)} \mathcal{B}_{W}^{(1)}+\sum_{i<j} \mathcal{E}_{i}^{(1)} \mathcal{E}_{j}^{(1)}+\mathcal{E}^{(1)} V^{(1)}-\left(\mathcal{E}^{(1)}+V^{(1)}\right) \frac{A_{W W}^{(1)}}{M_{W}^{2}} .
$$

The indices $i, j$ in eq. (2.32) label the different species in the muon decay: $\mu, e, \nu_{\mu}$ and $\nu_{e}$ with the sum that runs over $i<j$ because the terms with $i=j$ are included in $\mathcal{E}^{(2)}$.

We recall that $\Delta r_{0}$ is an infrared (IR) safe quantity but not ultraviolet (UV) finite. However, the $\mathcal{E}$ and $\mathcal{B}_{W}$ terms in eq. (2.29) and (2.30) contain IR-divergent contributions from photon diagrams. To separate the UV-divergent terms from the IR ones we regulated the latter giving a small mass to the photon. We then explicitly verified the cancellation of all IR divergent contributions.

The other proper two-loop contributions to $\lambda_{\mathrm{EW}}^{(2)}(\bar{\mu})$ are the two-loop tadpole diagrams and the two-loop Higgs boson mass counterterm. The Higgs mass counterterm, not taking into account negligible width effects, is given by

$$
\delta M_{h}^{2}=\operatorname{Re} \Pi_{h h}\left(M_{h}^{2}\right)
$$

with $\Pi_{h h}\left(M_{h}^{2}\right)$ the Higgs self-energy evaluated at external momentum equal to $M_{h}$. The Higgs mass counterterm as defined in eq. (2.33) is a gauge-dependent quantity. Yet, as proved at the beginning of section $2, \lambda(\bar{\mu})$ is a gauge-invariant object.

The diagrams contributing to $\delta^{(2)} \lambda_{\mathrm{OS}}$ were generated using the Mathematica package FEynaRTs [104]. The reduction of the two-loop diagrams to scalar integrals was done 
using the code TARCER [105] that uses the Tarasov's algorithm [106] and it is now part of the FeynCALC [107] package. In order to extract the $V_{W}$ and $\mathcal{B}_{W}$ terms in $\Delta r_{0}$ from the relevant diagrams we used the projector presented in ref. [108]. The two-loop self-energy diagrams at external momenta different from zero were reduced to the set of loop-integral basis functions introduced in ref. [109]. The evaluation of the basis functions was done numerically using the code TSIL [110].

The two loop correction to $\lambda$ is the sum of a QCD term and of an electroweak (EW) term. The QCD correction $\lambda_{\mathrm{QCD}}^{(2)}(\bar{\mu})$ is reported as an approximated formula in eq. (47) of [8]. For simplicity here we present it also in a numerical form:

$$
\lambda_{\mathrm{QCD}}^{(2)}\left(\bar{\mu}=M_{t}\right)=\frac{g_{3}^{2}}{(4 \pi)^{4}}\left[-23.89+0.12\left(\frac{M_{h}}{\mathrm{GeV}}-125\right)-0.64\left(\frac{M_{t}}{\mathrm{GeV}}-173\right)\right] .
$$

The result for $\lambda_{\mathrm{EW}}^{(2)}(\bar{\mu})$ is too long to be displayed explicitly. Here we present it in a numerical form valid around the measured values of $M_{h}$ and $M_{t}$. Using the inputs in table 2 we find

$$
\lambda_{\mathrm{EW}}^{(2)}\left(\bar{\mu}=M_{t}\right)=\frac{1}{(4 \pi)^{4}}\left[-9.45-0.11\left(\frac{M_{h}}{\mathrm{GeV}}-125\right)-0.21\left(\frac{M_{t}}{\mathrm{GeV}}-173\right)\right] .
$$

The numerical expression in eq. (2.35) is accidentally very close to the gaugeless limit of the SM presented in eq. (2.45) of [8]. Furthermore, as a check of our result, we verified that in the (physically irrelevant) limit $M_{h}=0$, it agrees with an independent computation of $\lambda^{(2)}$ performed using the known results for the two-loop effective potential in the Landau gauge.

\subsection{Two-loop correction to the Higgs mass term}

The result for the mass term in the Higgs potential can be easily obtained from that on $\lambda(\bar{\mu})$. We write

$$
m^{2}(\bar{\mu})=M_{h}^{2}+\delta^{(1)} m^{2}(\bar{\mu})+\delta^{(2)} m^{2}(\bar{\mu})
$$

with

$$
\begin{aligned}
& \delta^{(1)} m^{2}(\bar{\mu})=-\left.\delta^{(1)} m_{\mathrm{OS}}^{2}\right|_{\mathrm{fin}}, \\
& \delta^{(2)} m^{2}(\bar{\mu})=-\left.\delta^{(2)} m_{\mathrm{OS}}^{2}\right|_{\mathrm{fin}}+\Delta_{m^{2}}
\end{aligned}
$$

The one-loop contribution in eq. $(2.36), \delta^{(1)} m^{2}(\bar{\mu})$, is given by the finite part of eq. (2.15). The two-loop corrections in eq. $(2.36), \delta^{(2)} m^{2}(\bar{\mu})$, can be divided into a QCD contribution plus an EW contribution.

The QCD contribution, $\delta_{\mathrm{QCD}}^{(2)} m^{2}(\bar{\mu})$, can be obtained evaluating the relevant diagrams via a Taylor series in $x_{h t} \equiv M_{h}^{2} / M_{t}^{2}$ up to fourth order

$$
\begin{aligned}
\delta_{\mathrm{QCD}}^{(2)} m^{2}(\bar{\mu})=\frac{G_{\mu} M_{t}^{4}}{\sqrt{2}(4 \pi)^{4}} N_{c} C_{F} g_{3}^{2}[ & -96+\left(41-12 \ln ^{2} \frac{M_{t}^{2}}{\bar{\mu}^{2}}+12 \ln ^{2} \frac{M_{t}^{2}}{\bar{\mu}^{2}}\right) x_{h t}+ \\
& \left.+\frac{122}{135} x_{h t}^{2}+\frac{1223}{3150} x_{h t}^{3}+\frac{43123}{661500} x_{h t}^{4}\right]
\end{aligned}
$$


where $N_{c}$ and $C_{F}$ are colour factors $\left(N_{c}=3, C_{F}=4 / 3\right)$, such that it is numerically approximated as

$$
\delta_{\mathrm{QCD}}^{(2)} m^{2}\left(\bar{\mu}=M_{t}\right)=\frac{g_{3}^{2} M_{h}^{2}}{(4 \pi)^{4}}\left[-140.50+2.89\left(\frac{M_{h}}{\mathrm{GeV}}-125\right)-3.71\left(\frac{M_{t}}{\mathrm{GeV}}-173\right)\right] .
$$

The two-loop EW part, $\delta_{\mathrm{EW}}^{(2)} m^{2}(\bar{\mu})$, can be obtained as a byproduct of the calculation of $\lambda_{\mathrm{EW}}^{(2)}(\bar{\mu})$. Also in this case the result is too long to be displayed and we present an interpolating formula. Using the inputs in table 2 we find

$$
\delta_{\mathrm{EW}}^{(2)} m^{2}\left(\bar{\mu}=M_{t}\right)=\frac{M_{h}^{2}}{(4 \pi)^{4}}\left[-149.47+2.53\left(\frac{M_{h}}{\mathrm{GeV}}-125\right)-4.69\left(\frac{M_{t}}{\mathrm{GeV}}-173\right)\right] .
$$

\subsection{Two loop correction to the top Yukawa coupling}

The $\overline{\mathrm{MS}}$ top Yukawa coupling is given by

$$
y_{t}(\bar{\mu})=2\left(\frac{G_{\mu}}{\sqrt{2}} M_{t}^{2}\right)^{1 / 2}+y_{t}^{(1)}(\bar{\mu})+y_{t}^{(2)}(\bar{\mu})
$$

with

$$
\begin{aligned}
& y_{t}^{(1)}(\bar{\mu})=-\left.\delta^{(1)} y_{t_{\mathrm{OS}}}\right|_{\mathrm{fin}}, \\
& y_{t}^{(2)}(\bar{\mu})=-\left.\delta^{(2)} y_{t_{\mathrm{OS}}}\right|_{\mathrm{fin}}+\Delta_{y_{t}} .
\end{aligned}
$$

According to eqs. (2.19)-(2.20) the corrections to the tree-level value of $y_{t}$ are given in terms of $\Delta r_{0}$ and the top mass counterterm. Regarding the latter, a general discussion on the mass counterterm for unstable fermions in parity-nonconserving theories is presented in ref. [111]. Writing the fermion self-energy as

$$
\begin{aligned}
\Sigma(p) & =\Sigma_{1}(p)+\Sigma_{2}(p) \gamma_{5}, \\
\Sigma_{1,2}(p) & =\not p B_{1,2}\left(p^{2}\right)+m_{0} A_{1,2}\left(p^{2}\right),
\end{aligned}
$$

the fermion propagator is given by

$$
i S(p)=\frac{i}{\not p-m_{0}-\Sigma(p)}=\frac{i}{\not p-m_{0}-\Sigma_{\mathrm{eff}}(p)}\left[1-\frac{\Sigma_{2}(p)}{\not p-\Sigma_{1}(p)+m_{0}\left[1+2 A_{1}\left(p^{2}\right)\right]} \gamma_{5}\right]
$$

where $m_{0}$ is the bare fermion mass and

$$
\Sigma_{\text {eff }}(p)=\Sigma_{1}(p)+\frac{\Sigma_{2}(p)\left[\Sigma_{2}(p)-2 m_{0} A_{2}\left(p^{2}\right)\right]}{\not p-\Sigma_{1}(p)+m_{0}\left[1+2 A_{1}\left(p^{2}\right)\right]} .
$$

Identifying the position $\not p=\tilde{M}$ of the complex pole in eq. (2.44) by

$$
\tilde{M}=m_{0}+\Sigma_{\text {eff }}(\tilde{M})
$$

and parametrizing $\tilde{M}=M-i \Gamma / 2$ with $M$ the pole mass of the unstable fermion and $\Gamma$ its width, the mass counterterm for the unstable fermion is found to be

$$
\delta M=\operatorname{Re} \Sigma_{\text {eff }}(\tilde{M}) .
$$


Specialising the above discussion to the top, we find, including up to two-loop contributions,

$$
\delta M_{t}=\operatorname{Re}\left[\Sigma_{1}\left(\widetilde{M}_{t}\right)+\frac{\Sigma_{2}\left(M_{t}\right)\left[\Sigma_{2}\left(M_{t}\right)-2 M_{t} A_{2}\left(M_{t}^{2}\right)\right]}{2 M_{t}}\right]
$$

with $\widetilde{M}_{t}=M_{t}-i \Gamma_{t} / 2$. The mass counterterm defined in eq. (2.48) is expressed in terms of the self-energy diagrams only, without including the tadpole contribution. While this definition follows from our choice of identifying the renormalised vacuum with the minimum of the radiatively corrected potential, it gives rise to a $\delta M_{t}$ that is gauge-dependent and, as a consequence, in this framework, the $\overline{\mathrm{MS}}$ top mass, $M_{t}(\bar{\mu})$, is a gauge-dependent quantity. However, a $\overline{\mathrm{MS}}$ mass is not a physical quantity nor a Lagrangian parameter and therefore the requirement of gauge-invariance is not mandatory. A gauge-invariant definition of $M_{t}(\bar{\mu})$ can be obtained by including the tadpole contribution in the mass counterterm [86]. However, with this choice the relation between the pole and $\overline{\mathrm{MS}}$ masses of top quark acquires a very large electroweak correction [112]. The top Yukawa coupling computed in this paper is a parameter of the Lagrangian, and thereby does not suffer of these problems.

Concerning the two-loop contributions in eq. (2.41), we have computed the QCD corrections to the one-loop term and the two-loop EW contribution.

These contributions are too long to be displayed explicitly, and we report them as interpolating formulæ. Using the inputs in table 2 we find

$$
\begin{aligned}
y_{t}^{(2)}\left(\bar{\mu}=M_{t}\right)= & \frac{1}{(4 \pi)^{4}}\left[5.22-0.01\left(\frac{M_{h}}{\mathrm{GeV}}-125\right)+0.15\left(\frac{M_{t}}{\mathrm{GeV}}-173\right)\right]+ \\
& +\frac{g_{3}^{2}}{(4 \pi)^{4}}\left[-7.53+0.09\left(\frac{M_{h}}{\mathrm{GeV}}-125\right)-0.23\left(\frac{M_{t}}{\mathrm{GeV}}-173\right)\right]+ \\
& +\frac{g_{3}^{4}}{(4 \pi)^{4}}\left[-145.08-0.84\left(\frac{M_{t}}{\mathrm{GeV}}-173\right)\right]
\end{aligned}
$$

where the last term is the well known pure QCD contribution; the second term is the mixed QCD/EW contribution that agrees with [38]; the first term is the pure EW contribution computed in this paper for the first time.

\subsection{Two-loop correction to weak and hypercharge gauge couplings}

The $g_{2}$ and $g_{Y}$ gauge couplings are given by

$$
\begin{aligned}
g_{2}(\bar{\mu}) & =2\left(\sqrt{2} G_{\mu}\right)^{1 / 2} M_{W}+g_{2}^{(1)}(\bar{\mu})+g_{2}^{(2)}(\bar{\mu}), \\
g_{Y}(\bar{\mu}) & =2\left(\sqrt{2} G_{\mu}\right)^{1 / 2} \sqrt{M_{Z}^{2}-M_{W}^{2}}+g_{Y}^{(1)}(\bar{\mu})+g_{Y}^{(2)}(\bar{\mu}),
\end{aligned}
$$

with

$$
\begin{array}{ll}
g_{2}^{(1)}(\bar{\mu})=-\left.\delta^{(1)} g_{2_{\mathrm{OS}}}\right|_{\text {fin }}, & g_{2}^{(2)}(\bar{\mu})=-\left.\delta^{(2)} g_{2_{\mathrm{OS}}}\right|_{\text {fin }}+\Delta_{g_{2}}, \\
g_{Y}^{(1)}(\bar{\mu})=-\left.\delta^{(1)} g_{Y_{\mathrm{OS}}}\right|_{\text {fin }}, & g_{Y}^{(2)}(\bar{\mu})=-\left.\delta^{(2)} g_{Y_{\mathrm{OS}}}\right|_{\text {fin }}+\Delta_{g_{y}} .
\end{array}
$$




\begin{tabular}{|c|ccccc|}
\hline $\bar{\mu}=M_{t}$ & $\lambda$ & $y_{t}$ & $g_{2}$ & $g_{Y}$ & $m / \mathrm{GeV}$ \\
\hline LO & 0.13023 & 0.99425 & 0.65294 & 0.34972 & 125.66 \\
NLO & 0.12879 & 0.94953 & 0.64755 & 0.35937 & 132.80 \\
NNLO & 0.12710 & 0.93849 & 0.64822 & 0.35760 & 131.99 \\
\hline
\end{tabular}

Table 3. Values of the fundamental SM parameters computed at tree level, one loop, two loops in the $\overline{\mathrm{MS}}$ scheme and renormalised at $\bar{\mu}=M_{t}$ for the central values of the measurements listed in table 2 .

The one-loop contributions in eq. (2.51) are given by the finite part of eqs. (2.21), (2.23). Also in this case the results of the two-loop corrections in eq. (2.51) are too long to be displayed and we present them with interpolating formulas. Using the inputs in table 2 we find

$$
\begin{aligned}
g_{2}^{(2)}\left(\bar{\mu}=M_{t}\right)= & \frac{1}{(4 \pi)^{4}}\left[5.83+0.01\left(\frac{M_{h}}{\mathrm{GeV}}-125\right)+0.01\left(\frac{M_{t}}{\mathrm{GeV}}-173\right)\right]+ \\
& +\frac{g_{3}^{2}}{(4 \pi)^{4}}\left[7.98+0.01\left(\frac{M_{t}}{\mathrm{GeV}}-173\right)\right]
\end{aligned}
$$

and

$$
\begin{aligned}
g_{Y}^{(2)}\left(\bar{\mu}=M_{t}\right)= & \frac{1}{(4 \pi)^{4}}\left[-12.58-0.01\left(\frac{M_{h}}{\mathrm{GeV}}-125\right)-0.11\left(\frac{M_{t}}{\mathrm{GeV}}-173\right)\right]+ \\
& +\frac{g_{3}^{2}}{(4 \pi)^{4}}\left[-23.12-0.14\left(\frac{M_{t}}{\mathrm{GeV}}-173\right)\right]
\end{aligned}
$$

\section{SM couplings at the electroweak scale}

In this section we give practical results for the SM parameters $\theta=\left\{\lambda, m^{2}, y_{t}, g_{2}, g_{Y}\right\}$ computed in terms of the observables $M_{h}, M_{t}, M_{W}, M_{Z}, G_{\mu}$ and $\alpha_{3}\left(M_{Z}\right)$, whose measured values are listed in table 2. Each $\overline{\mathrm{MS}}$ parameter $\theta$ is expanded in loops as

$$
\theta=\theta^{(0)}+\theta^{(1)}+\theta^{(2)}+\cdots
$$

where

1. the tree-level values $\theta^{(0)}$ are listed in table 1 ;

2. the one-loop corrections $\theta^{(1)}$ are analytically given in appendix A;

3. the two-loop corrections $\theta^{(2)}$ are computed in section 2 .

After combining these corrections, we give in the following the numerical values for the SM parameters renormalised at the top pole mass $M_{t}$ in the $\overline{\mathrm{MS}}$ scheme. 


\subsection{The Higgs quartic coupling}

For the Higgs quartic coupling, defined by writing the SM potential as $V=-\frac{1}{2} m^{2}|H|^{2}+$ $\lambda|H|^{4}$, we find

$\lambda\left(\bar{\mu}=M_{t}\right)=0.12711+0.00206\left(\frac{M_{h}}{\mathrm{GeV}}-125.66\right)-0.00004\left(\frac{M_{t}}{\mathrm{GeV}}-173.10\right) \pm 0.00030_{\mathrm{th}}$.

The dependence on $M_{t}$ is small because $\lambda$ is renormalised at $M_{t}$ itself. Here and below the theoretical uncertainty is estimated from the dependence on $\bar{\mu}$ (varied around $M_{t}$ by one order of magnitude) of the higher-order unknown 3 loop corrections. Such dependence is extracted from the known SM RGE at 3 loops (as summarized in appendix B). ${ }^{5}$

\subsection{The Higgs mass term}

For the mass term of the Higgs doublet in the SM Lagrangian (normalised such that $m=M_{h}$ at tree level) we find

$$
\frac{m\left(\bar{\mu}=M_{t}\right)}{\mathrm{GeV}}=132.03+0.94\left(\frac{M_{h}}{\mathrm{GeV}}-125.66\right)+0.17\left(\frac{M_{t}}{\mathrm{GeV}}-173.10\right) \pm 0.15_{\mathrm{th}} .
$$

\subsection{The top Yukawa coupling}

For the top Yukawa coupling we get

$$
\begin{aligned}
y_{t}\left(\bar{\mu}=M_{t}\right)= & 0.93558+0.00550\left(\frac{M_{t}}{\mathrm{GeV}}-173.10\right)+ \\
& -0.00042 \frac{\alpha_{3}\left(M_{Z}\right)-0.1184}{0.0007}-0.00042 \frac{M_{W}-80.384 \mathrm{GeV}}{0.014 \mathrm{GeV}} \pm 0.00050_{\mathrm{th}} .
\end{aligned}
$$

The central value differs from the NNLO value in table 3 because we include here also the NNNLO (3 loop) pure QCD effect [83-85]. The theoretical uncertainty is estimated accordingly, and does not take into account the non-perturbative theoretical uncertainty of order $\Lambda_{\mathrm{QCD}}$ in the definition of $M_{t}$.

\subsection{The weak gauge couplings}

For the weak gauge couplings $g_{2}$ and $g_{Y}$ computed at NNLO accuracy in terms of $M_{W}$ and $M_{Z}$ we find

$$
\begin{aligned}
& g_{2}\left(\bar{\mu}=M_{t}\right)=0.64822+0.00004\left(\frac{M_{t}}{\mathrm{GeV}}-173.10\right)+0.00011 \frac{M_{W}-80.384 \mathrm{GeV}}{0.014 \mathrm{GeV}}, \\
& g_{Y}\left(\bar{\mu}=M_{t}\right)=0.35761+0.00011\left(\frac{M_{t}}{\mathrm{GeV}}-173.10\right)-0.00021 \frac{M_{W}-80.384 \mathrm{GeV}}{0.014 \mathrm{GeV}},
\end{aligned}
$$

where the adopted value for $M_{W}$ and its experimental error are reported in table 2.

\footnotetext{
${ }^{5}$ Recently the calculation of the three-loop SM effective potential at leading order in strong and top Yukawa couplings has appeared [113]. The resulting three-loop contributions to the Higgs quartic coupling are within our estimated error. Combining the three loop effective potential with 1 and 2-loop renormalizations, we extract the 3-loop pure QCD correction to $\lambda\left(\bar{\mu}=M_{t}\right)$ in the limit $M_{h}, M_{W}, M_{Z} \ll M_{t}$ :

$\lambda_{\mathrm{QCD}}^{(3)}=-\frac{8}{135} \frac{g_{3}^{4} G_{\mu}^{2} M_{t}^{4}}{(4 \pi)^{6}}\left[176 \pi^{4}+240 \pi^{2}\left(3+4 \ln ^{2} 2+6 \ln 2\right)+15\left(607-64 \ln ^{4} 2-1536 \mathrm{Li}_{4}\left(\frac{1}{2}\right)+576 \zeta(3)\right]\right.$.
} 


\subsection{The strong gauge coupling}

Table 2 contains the value of $\alpha_{3}\left(M_{Z}\right)$, as extracted from the global fit of [101] in the effective SM with 5 flavours. Including RG running from $M_{Z}$ to $M_{t}$ at 4 loops in QCD and at 2 loops in the electroweak gauge interactions, and 3 loop QCD matching at $M_{t}$ to the full SM with 6 flavours, we get

$$
g_{3}\left(\bar{\mu}=M_{t}\right)=1.1666+0.00314 \frac{\alpha_{3}\left(M_{Z}\right)-0.1184}{0.0007}-0.00046\left(\frac{M_{t}}{\mathrm{GeV}}-173.10\right) .
$$

The SM parameters can be renormalised to any other desired energy by solving the SM renormalisation group equations summarised in appendix B. For completeness, we include in the one- and two-loop RG equations the contributions of the small bottom and tau Yukawa couplings, as computed from the $\overline{\mathrm{MS}} b$-quark mass, $m_{b}\left(m_{b}\right)=4.2 \mathrm{GeV}$, and from $M_{\tau}=1.777 \mathrm{GeV}$. Within the $\overline{\mathrm{MS}}$ scheme $\beta$ functions are gauge-independent [88, 89]; similarly the $\overline{\mathrm{MS}}$ parameters are gauge independent too.

\section{Extrapolation of the SM up to the Planck scale}

The most puzzling and intriguing outcome of the Higgs discovery has been the finding that $M_{h}$ lies very close to the boundary between stability and metastability regions. This result is the main motivation for our refined NNLO calculation of the SM Higgs potential at large field values. Indeed, the special Higgs mass found by ATLAS and CMS is so close to criticality that any statement about stability or metastability of the EW vacuum requires a careful analysis of theoretical and experimental errors. The discovered proximity to criticality also naturally stimulates many theoretical speculations on its possible hidden significance or on special matching conditions at very high energy scales. In the rest of the paper, we will explore the implications of our improved computation of the Higgs quartic coupling extrapolated to very high scales.

\subsection{SM couplings at the Planck scale}

The first issue we want to address concerns the size of the SM coupling constants. When we try to extract information from the values of the coupling constants, it is reasonable to analyse their values not at the weak scale, but at some high-energy scale where we believe the SM matches onto some extended theory. So, using our NNLO results, we extrapolate the SM couplings from their weak-scale values (as determined in section 3) to higher energies.

The evolution of the SM couplings up to a large cut-off scale is shown in figure 1. At the Planck mass, we find the following values of the SM parameters:

$$
\begin{aligned}
& g_{1}\left(M_{\mathrm{Pl}}\right)=0.6133+0.0003\left(\frac{M_{t}}{\mathrm{GeV}}-173.10\right)-0.0006 \frac{M_{W}-80.384 \mathrm{GeV}}{0.014 \mathrm{GeV}} \\
& g_{2}\left(M_{\mathrm{Pl}}\right)=0.5057 \\
& g_{3}\left(M_{\mathrm{Pl}}\right)=0.4873+0.0002 \frac{\alpha_{3}\left(M_{Z}\right)-0.1184}{0.0007} \\
& y_{t}\left(M_{\mathrm{Pl}}\right)=0.3813+0.0051\left(\frac{M_{t}}{\mathrm{GeV}}-173.10\right)-0.0021 \frac{\alpha_{3}\left(M_{Z}\right)-0.1184}{0.0007}
\end{aligned}
$$




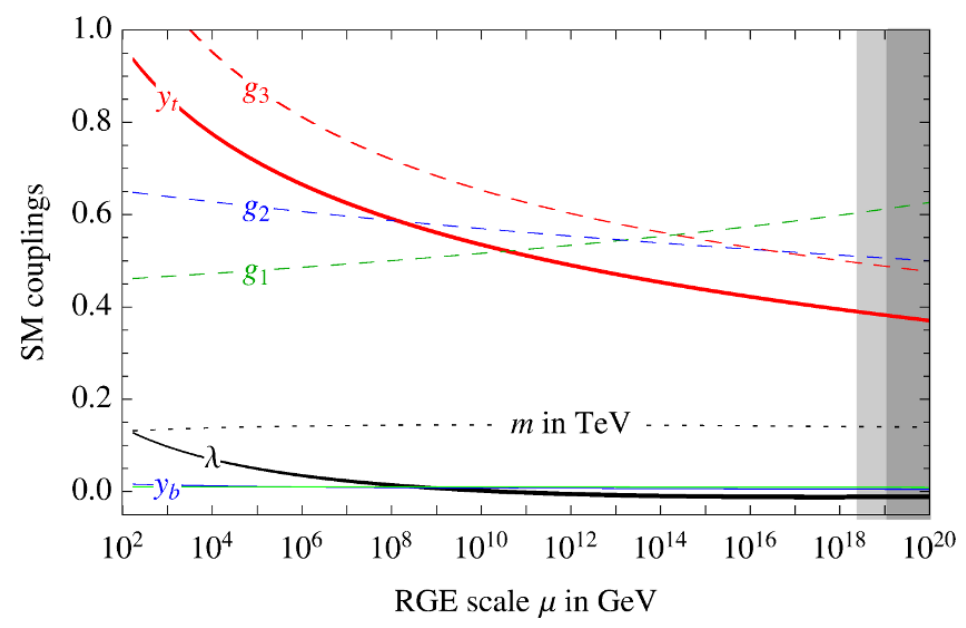

Figure 1. Renormalisation of the SM gauge couplings $g_{1}=\sqrt{5 / 3} g_{Y}, g_{2}, g_{3}$, of the top, bottom and $\tau$ couplings $\left(y_{t}, y_{b}, y_{\tau}\right)$, of the Higgs quartic coupling $\lambda$ and of the Higgs mass parameter $m$. All parameters are defined in the $\overline{\mathrm{MS}}$ scheme. We include two-loop thresholds at the weak scale and three-loop RG equations. The thickness indicates the $\pm 1 \sigma$ uncertainties in $M_{t}, M_{h}, \alpha_{3}$.

$$
\begin{aligned}
\lambda\left(M_{\mathrm{Pl}}\right)= & -0.0113-0.0065\left(\frac{M_{t}}{\mathrm{GeV}}-173.10\right)+ \\
& +0.0018 \frac{\alpha_{3}\left(M_{Z}\right)-0.1184}{0.0007}+0.0029\left(\frac{M_{h}}{\mathrm{GeV}}-125.66\right) \\
m\left(M_{\mathrm{Pl}}\right)= & 140.3 \mathrm{GeV}+1.6 \mathrm{GeV}\left(\frac{M_{h}}{\mathrm{GeV}}-125.66\right)+ \\
& -0.25 \mathrm{GeV}\left(\frac{M_{t}}{\mathrm{GeV}}-173.10\right)+0.05 \mathrm{GeV} \frac{\alpha_{3}\left(M_{Z}\right)-0.1184}{0.0007}
\end{aligned}
$$

All Yukawa couplings, other than the one of the top quark, are very small. This is the well-known flavour problem of the SM, which will not be investigated in this paper.

The three gauge couplings and the top Yukawa coupling remain perturbative and are fairly weak at high energy, becoming roughly equal in the vicinity of the Planck mass. The near equality of the gauge couplings may be viewed as an indicator of an underlying grand unification even within the simple SM, once we allow for threshold corrections of the order of $10 \%$ around a scale of about $10^{16} \mathrm{GeV}$ (of course, in the spirit of this paper, we are disregarding the acute naturalness problem). It is amusing to note that the ordering of the coupling constants at low energy is completely overturned at high energy. The (properly normalised) hypercharge coupling $g_{1}$ becomes the largest coupling in the SM already at scales of about $10^{14} \mathrm{GeV}$, and the weak coupling $g_{2}$ overcomes the strong coupling at about $10^{16} \mathrm{GeV}$. The top Yukawa becomes smaller than any of the gauge couplings at scales larger than about $10^{10} \mathrm{GeV}$.

The Higgs quartic coupling remains weak in the entire energy domain below $M_{\mathrm{Pl}}$. It decreases with energy crossing $\lambda=0$ at a scale of about $10^{10} \mathrm{GeV}$, see figure 2 (upper 

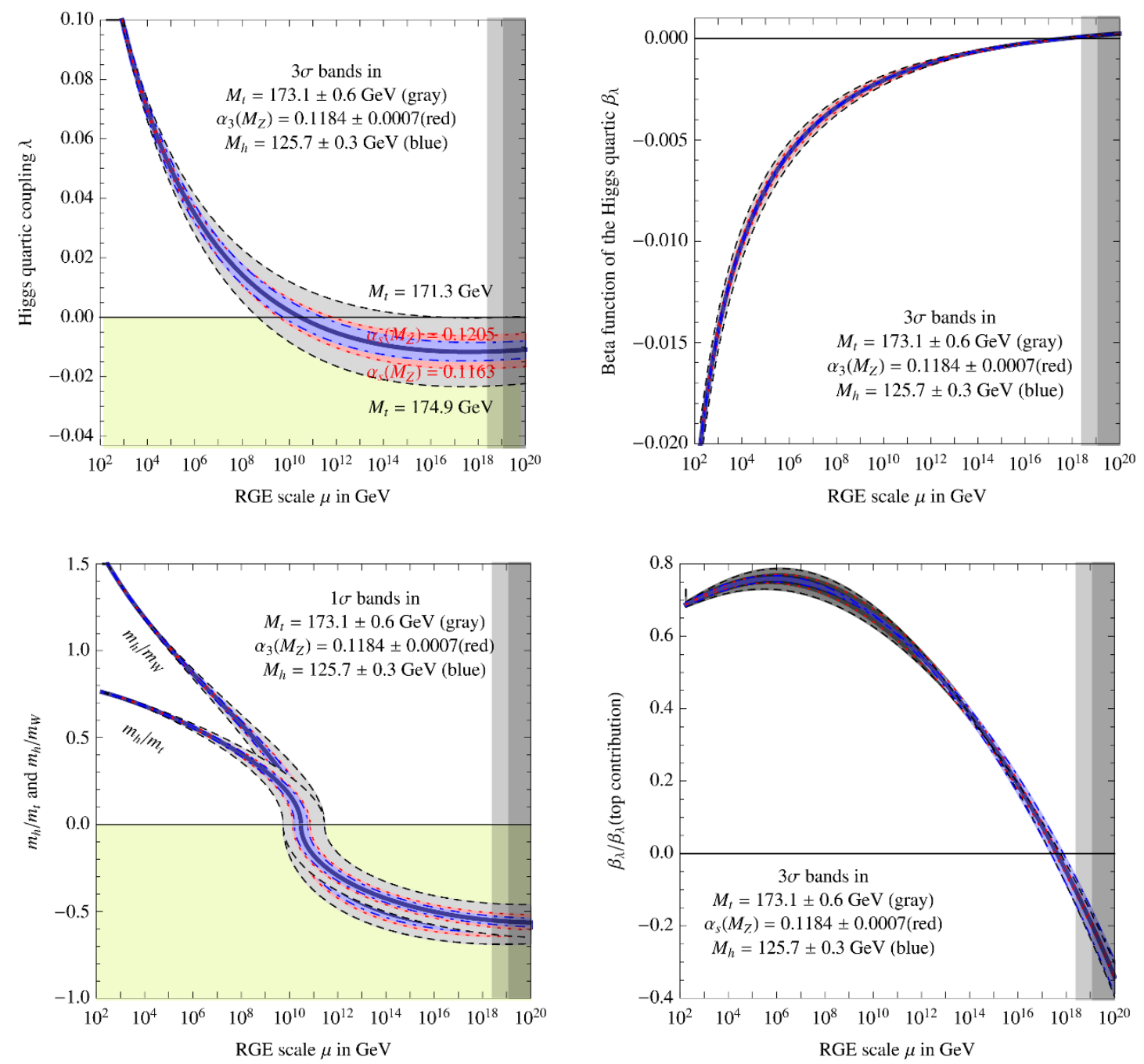

Figure 2. Upper: RG evolution of $\lambda$ (left) and of $\beta_{\lambda}$ (right) varying $M_{t}, \alpha_{3}\left(M_{Z}\right), M_{h}$ by $\pm 3 \sigma$. Lower: same as above, with more "physical" normalisations. The Higgs quartic coupling is compared with the top Yukawa and weak gauge coupling through the ratios $\operatorname{sign}(\lambda) \sqrt{4|\lambda|} / y_{t}$ and $\operatorname{sign}(\lambda) \sqrt{8|\lambda|} / g_{2}$, which correspond to the ratios of running masses $m_{h} / m_{t}$ and $m_{h} / m_{W}$, respectively (left). The Higgs quartic $\beta$-function is shown in units of its top contribution, $\beta_{\lambda}$ (top contribution) $=-3 y_{t}^{4} / 8 \pi^{2}$ (right). The grey shadings cover values of the RG scale above the Planck mass $M_{\mathrm{Pl}} \approx 1.2 \times 10^{19} \mathrm{GeV}$, and above the reduced Planck mass $\bar{M}_{\mathrm{Pl}}=M_{\mathrm{Pl}} / \sqrt{8 \pi}$.

left). Indeed, $\lambda$ is the only SM coupling that is allowed to change sign during the RG evolution because it is not multiplicatively renormalised. For all other SM couplings, the $\beta$ functions are proportional to their respective couplings and crossing zero is not possible. This corresponds to the fact that $\lambda=0$ is not a point of enhanced symmetry.

In figure 2 (lower left) we compare the size of $\lambda$ with the top Yukawa coupling $y_{t}$ and the gauge coupling $g_{2}$, choosing a normalisation such that each coupling is equal to the corresponding particle mass, up to the same proportionality constant. In other words, we 
are plotting the ratios

$$
\operatorname{sign}(\lambda) \times \sqrt{4|\lambda|} / y_{t} \quad \text { and } \quad \operatorname{sign}(\lambda) \times \sqrt{8|\lambda|} / g_{2},
$$

equal to the ratios of running masses $m_{h} / m_{t}$ and $m_{h} / m_{W}$, respectively. Except for the region in which $\lambda$ vanishes, the Higgs quartic coupling looks fairly "normal" with respect to the other SM couplings. Nonetheless, the RG effect reduces significantly the overall size of $\lambda$ in its evolution from low to high energy. Although the central values of Higgs and top masses do not favour a scenario with vanishing Higgs self coupling at the Planck scale $\left(M_{\mathrm{Pl}}\right)$ - a possibility originally proposed in ref. $[116,117]$ and discussed more recently in ref. $[8,118-121]$ - the smallness of $\lambda$ around $M_{\mathrm{Pl}}$ offers reasons for speculation, as we will discuss later.

Another important feature of the RG evolution of $\lambda$ is the slowing down of the running at high energy. As shown in figure 2 (upper right), the corresponding Higgs quartic $\beta$ function vanishes at a scale of about $10^{17}-10^{18} \mathrm{GeV}$. In order to quantify the degree of cancellation in the $\beta$-function, we plot in figure 2 (lower right) $\beta_{\lambda}$ in units of its pure top contribution. The vanishing of $\beta_{\lambda}$ looks more like an accidental cancellation between various large contributions, rather than an asymptotic approach to zero. Given that the $\beta$-functions of the other SM couplings are all different than zero, it is not evident to find valid symmetry or dynamical reasons for the vanishing of $\beta_{\lambda}$ alone near $M_{\mathrm{Pl}}$. However, the smallness of $\beta_{\lambda}$ (and $\lambda$ ) at high energy implies that tiny variations of the input values of the couplings at $M_{\mathrm{Pl}}$ lead to wide fluctuations of the instability scale, thus justifying our refined calculation.

\subsection{Derivation of the stability bound}

In order to compute the stability bound on the Higgs mass one has to study the full effective potential and identify the critical Higgs field above which the potential becomes smaller than the value at the EW vacuum. We will refer to such critical energy as the instability scale $\Lambda_{I}$.

A first estimate of the instability scale can be obtained by approximating the effective potential with its RG-improved tree level expression. The analysis shows that the instability scale occurs at energies much bigger than the EW scale. Thus, for our purposes, the approximation of neglecting $v$ with respect to the value of the field $h$ is amply justified. Under this assumption, the effective potential (in the relevant region $h \gg v$ ) becomes

$$
V_{\text {eff }}(h)=\lambda_{\text {eff }}(h) \frac{h^{4}}{4} .
$$

The quantity $\lambda_{\text {eff }}$ can be extracted from the effective potential at two loops [122] and is explicitly given in appendix $\mathrm{C}$.

\subsection{The SM phase diagram in terms of Higgs and top masses}

The two most important parameters that determine the various EW phases of the SM are the Higgs and top-quark masses. In figure 3 we update the phase diagram given in ref. [8] with our improved calculation of the evolution of the Higgs quartic coupling. The regions of 

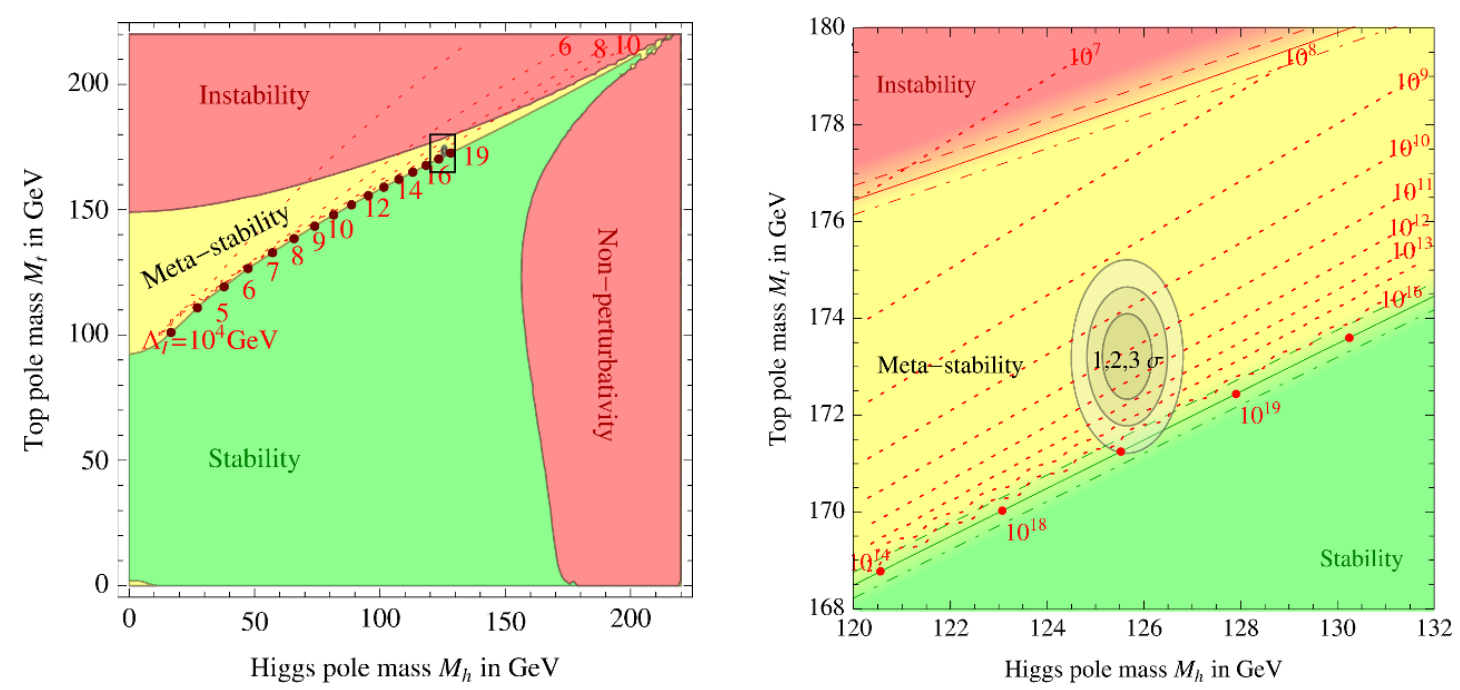

Figure 3. Left: SM phase diagram in terms of Higgs and top pole masses. The plane is divided into regions of absolute stability, meta-stability, instability of the SM vacuum, and non-perturbativity of the Higgs quartic coupling. The top Yukawa coupling becomes non-perturbative for $M_{t}>230 \mathrm{GeV}$. The dotted contour-lines show the instability scale $\Lambda_{I}$ in $\mathrm{GeV}$ assuming $\alpha_{3}\left(M_{Z}\right)=0.1184$. Right: zoom in the region of the preferred experimental range of $M_{h}$ and $M_{t}$ (the grey areas denote the allowed region at 1,2 , and $3 \sigma$ ). The three boundary lines correspond to 1- $\sigma$ variations of $\alpha_{3}\left(M_{Z}\right)=0.1184 \pm 0.0007$, and the grading of the colours indicates the size of the theoretical error.

stability, metastability, and instability of the EW vacuum are shown both for a broad range of $M_{h}$ and $M_{t}$, and after zooming into the region corresponding to the measured values. The uncertainty from $\alpha_{3}$ and from theoretical errors are indicated by the dashed lines and the colour shading along the borders. Also shown are contour lines of the instability scale $\Lambda_{I}$.

As previously noticed in ref. [8], the measured values of $M_{h}$ and $M_{t}$ appear to be rather special, in the sense that they place the SM vacuum in a near-critical condition, at the border between stability and metastability. In the neighbourhood of the measured values of $M_{h}$ and $M_{t}$, the stability condition is well approximated by

$$
M_{h}>129.1 \mathrm{GeV}+2.0\left(M_{t}-173.10 \mathrm{GeV}\right)-0.5 \mathrm{GeV} \frac{\alpha_{3}\left(M_{Z}\right)-0.1184}{0.0007} \pm 0.3 \mathrm{GeV}
$$

The quoted uncertainty comes only from higher order perturbative corrections. Other non-perturbative uncertainties associated with the relation between the measured value of the top mass and the actual definition of the top pole mass used here (presumably of the order of $\Lambda_{\mathrm{QCD}}$ ) are buried inside the parameter $M_{t}$ in eq. (4.4). For this reason we include a theoretical error in the top pole mass and take $M_{t}=\left(173.10 \pm 0.59_{\exp } \pm 0.3_{\mathrm{th}}\right) \mathrm{GeV}$. Combining in quadrature theoretical uncertainties with experimental errors, we find

$$
M_{h}>(129.1 \pm 1.5) \mathrm{GeV} \quad \text { (stability condition). }
$$


From this result we conclude that vacuum stability of the SM up to the Planck scale is excluded at $2.2 \sigma$ (98.6\% C.L. one-sided). Since the main source of uncertainty in eq. (4.4) comes from $M_{t}$, any refinement in the measurement of the top mass is of great importance for the question of EW vacuum stability.

Since the experimental error on the Higgs mass is already fairly small and will be further reduced by future LHC analyses, it is becoming more appropriate to express the stability condition in terms of the pole top mass. We can express the stability condition of eq. (4.4) as

$$
M_{t}<\left(171.53 \pm 0.15 \pm 0.23_{\alpha_{3}} \pm 0.15_{M_{h}}\right) \mathrm{GeV}=(171.53 \pm 0.42) \mathrm{GeV} .
$$

In the latter equation we combined in quadrature the theoretical uncertainty with the experimental uncertainties on $M_{h}$ and $\alpha_{3}$.

Notice that the stability bound is scheme and gauge independent. While intermediate steps of the computation (threshold corrections, higher-order RG equations, and the effective potential) are scheme-dependent, the values of the effective potential at its local minima are scheme-independent physical observables, and thus the stability condition has the same property.

We find that the instability scale (defined as the scale at which $\lambda_{\text {eff }}$ vanishes) is

$$
\log _{10} \frac{\Lambda_{I}}{\mathrm{GeV}}=11.3+1.0\left(\frac{M_{h}}{\mathrm{GeV}}-125.66\right)-1.2\left(\frac{M_{t}}{\mathrm{GeV}}-173.10\right)+0.4 \frac{\alpha_{3}\left(M_{Z}\right)-0.1184}{0.0007} .
$$

The scale $\Lambda_{0}$ at which the $\overline{\mathrm{MS}}$ running coupling $\lambda$ vanishes is a scheme-dependent quantity and is slightly smaller than the scale $\Lambda_{I}$. We find $\Lambda_{0} \approx 0.15 \Lambda_{I}$, with the same dependence on the SM parameters as in eq. (4.7). Even if $\Lambda_{I}$ is much smaller than $M_{\mathrm{Pl}}$, new physics at the Planck scale can affect the stability condition [57].

\subsection{The SM phase diagram in terms of Planck-scale couplings}

The discovery of the SM near-criticality has led to many theoretical speculations $[8,31-55$, $120,121]$. In order to address such speculations and to investigate if the measured value of $M_{h}$ is really special in the SM, it is more appropriate to study the phase diagram in terms of the Higgs quartic and the top Yukawa coupling evaluated at some high-energy scale, rather than at the weak scale. This is because of our theoretical bias that the SM is eventually embedded into a new framework at short distances, possibly as short as the Planck length. Therefore, it is more likely that information about the underlying theory is directly encoded in the high-energy coupling constants. For this reason in figure 4 we recast the phase diagram of figure 3 in terms of $\lambda\left(M_{\mathrm{Pl}}\right)$ and $y_{t}\left(M_{\mathrm{Pl}}\right)$. The diagram is shown in a broad range of couplings allowed by perturbativity, and also after zooming into the interesting region. The new area denoted as 'no EW vacuum' corresponds to a situation in which $\lambda$ is negative at the weak scale, and therefore the usual Higgs vacuum does not exist. In the region denoted as 'Planck-scale dominated' the instability scale $\Lambda_{I}$ is larger than $10^{18} \mathrm{GeV}$. In this situation we expect that both the Higgs potential and the tunnelling rate receive large gravitational corrections and any assessment about vacuum stability becomes unreliable.

From the left panel of figure 4 it is evident that, even when we consider the situation in terms of high-energy couplings, our universe appears to live under very special conditions. 

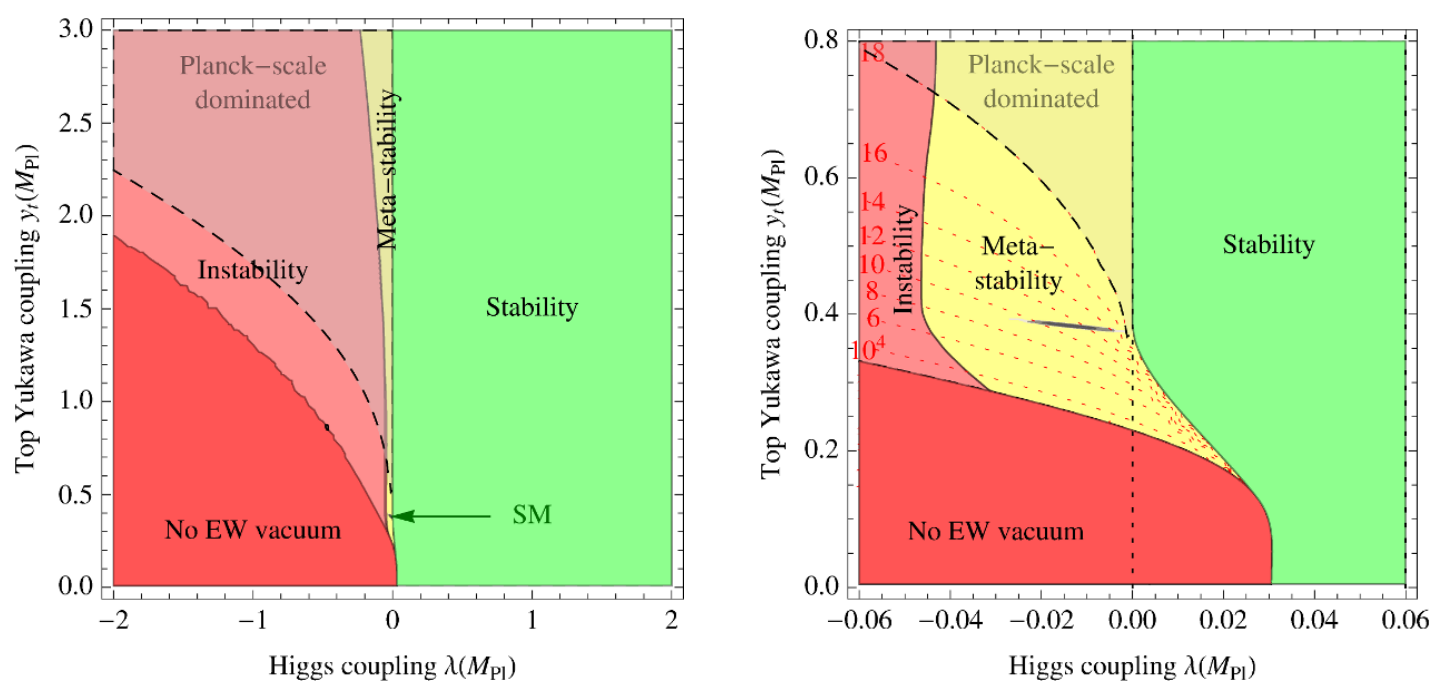

Figure 4. Left: SM phase diagram in terms of quartic Higgs coupling $\lambda$ and top Yukawa coupling $y_{t}$ renormalised at the Planck scale. The region where the instability scale $\Lambda_{I}$ is larger than $10^{18} \mathrm{GeV}$ is indicated as 'Planck-scale dominated'. Right: zoom around the experimentally measured values of the couplings, which correspond to the thin ellipse roughly at the centre of the panel. The dotted lines show contours of $\Lambda_{I}$ in $\mathrm{GeV}$.

The interesting theoretical question is to understand if the apparent peculiarity of $\lambda\left(M_{\mathrm{Pl}}\right)$ and $y_{t}\left(M_{\mathrm{Pl}}\right)$ carry any important information about phenomena well beyond the reach of any collider experiment. Of course this result could be just an accidental coincidence, because in reality the SM potential is significantly modified by new physics at low or intermediate scales. Indeed, the Higgs naturalness problem corroborates this possibility. However, both the reputed violation of naturalness in the cosmological constant and the present lack of new physics at the LHC cast doubts on the validity of the naturalness criterion for the Higgs boson. Of course, even without a natural EW sector, there are good reasons to believe in the existence of new degrees of freedom at intermediate energies. Neutrino masses, dark matter, axion, inflation, baryon asymmetry provide good motivations for the existence of new dynamics below the Planck mass. However, for each of these problems we can imagine solutions that either involve physics well above the instability scale or do not significantly modify the shape of the Higgs potential. As a typical example, take the see-saw mechanism. As shown in ref. [33], for neutrino masses smaller than $0.1 \mathrm{eV}$ (as suggested by neutrino-oscillation data without mass degeneracies), either neutrino Yukawa couplings are too small to modify the running of $\lambda$ or the right-handed neutrino masses are larger than the instability scale. In other words, a see-saw neutrino does not modify our conclusions about stability of the EW vacuum. Couplings of weak-scale dark matter to the Higgs boson are constrained to be small by WIMP direct searches (although dark-matter particles with weak interactions would modify the running of the weak gauge couplings, making the Higgs potential more stable). 
Thus, it is not inconceivable that the special values of $\lambda\left(M_{\mathrm{Pl}}\right)$ and $y_{t}\left(M_{\mathrm{Pl}}\right)$ carry a significance and it is worth to investigate their consequences. In the next section we discuss several possible classes of solutions that explain the apparent peculiarity of the SM parameters.

Finally, we notice that extrapolating SM parameters above the Planck scale ignoring gravity (this is a questionable assumption) the hypercharge couplings hits a Landau pole at about $10^{42} \mathrm{GeV}$. Demanding perturbativity up to such scale (rather than up to the Planck scale), the bounds on the top and Higgs masses become stronger by about 10 and $20 \mathrm{GeV}$ stronger respectively, and their measured values still lie in the region that can be extrapolated up to such high scale.

\section{Interpretations of the high-energy SM couplings}

The first possible interpretation, discussed in section 5.1, is the result of new dynamics occurring at some high-energy scale, the others find their most natural implementations in the multiverse.

\subsection{Matching conditions}

The special value of the Higgs quartic coupling could be the result of a matching condition with some high-energy theory in the vicinity of $M_{\mathrm{Pl}}$. It is not difficult to imagine theories able to drive $\lambda\left(M_{\mathrm{Pl}}\right)$ to zero: high-scale supersymmetry with $\tan \beta=1$ [123-128]; partial $N=2$ supersymmetry insuring $D$-flatness [129, 130]; an approximate Goldstone or shift symmetry [131, 132]; an infrared fixed-point of some transplanckian physics [121]; a powerlaw running in a quasi-conformal theory. Present data suggest that an exact zero of $\lambda$ is reached at scales of about $10^{10}-10^{12} \mathrm{GeV}$, see eq. (4.7), well below the Planck mass. It is not difficult to imagine theories that give $\lambda\left(M_{\mathrm{Pl}}\right)$ in agreement with eq. (4.1e) as a result of a vanishing matching condition modified by threshold corrections.

Supersymmetry is probably one of the best candidates able to explain the vanishing of $\lambda$ as a high-energy boundary condition, because of the natural appearance of radiativelystable flat directions. Such flat directions give a well-grounded justification for scalar particles with vanishing potentials, and yet interacting at zero momentum (contrary to the case of Goldstone bosons).

Note also that the smallness of the Higgs quartic $\beta$-function at high energy is the key ingredient that allows for the possibility of extending the SM up to a matching scale much larger than $\Lambda_{I}$. If $\lambda$ ran fast above $\Lambda_{I}$, it would rapidly trigger vacuum instability and the region of metastability would be limited to SM cut-off scales only slightly larger than $\Lambda_{I}$. This is another peculiarity of the measured values of $M_{h}$ and $M_{t}$.

\subsection{Criticality as an attractor}

Statistical properties of the multiverse offer alternatives to dynamical determinations of $\lambda\left(M_{\mathrm{Pl}}\right)$ from matching conditions with new theories. The first possibility we consider is motivated by the observation that the measured value of $M_{h}$ looks special, in the sense that it corresponds to a near-critical parameter separating two phases. As remarked in ref. [133], 
also Higgs naturalness can be viewed as a problem of near-criticality between two phases (i.e. why is the Higgs bilinear carefully selected just to place our universe at the edge between the broken and unbroken EW phases?). This leads to the speculation that, within the multiverse, critical points are attractors. If this vision is correct, the probability density in the multiverse is peaked around the boundaries between different phases, and generic universes are likely to live near critical lines. Then, near-criticality would be the result of probability distributions in the multiverse, and would not necessarily follow from anthropic considerations. In this picture, the Higgs parameters found in our universe are not at all special. On the contrary, they correspond to the most likely occurrence in the multiverse.

There are many natural phenomena in which near-criticality emerges as an attractor [134]. A typical example is given by the slope angle of sand dunes. While one could expect to find in a beach sand dunes with any possible slope angles, in practice the vast majority of dunes have a slope angle roughly equal to the so-called "angle of repose". The angle of repose is the steepest angle of descent, which is achieved when the material forming the pile is at a critical condition on the verge of sliding. The angle of repose depends on size and shape of the material granularity, and for sand is usually about 30-35 degrees. The typicality of finding sand dunes with slope angles near the critical value is simply understood in terms of the forces that shape dunes. Wind builds up the dune moving sand up to the top; gravity makes the pile collapse under its own weight when the dune is too steep. As a result, near-criticality is the most likely condition, as a compromise between two competing effects.

Something similar could happen with the Higgs parameters in the multiverse. Suppose that the probability distribution of the Higgs quartic coupling in the multiverse is not uniform, but is a monotonically decreasing function of $\lambda$. In other words, there is a pressure in the multiverse towards the smallest (possibly negative) $\lambda$. However, in universes where $\lambda$ is sufficiently negative, the Higgs field is destabilised, forming a bubble of AdS space with a negative cosmological constant of order $-M_{\mathrm{Pl}}^{4}$ in its interior. Such regions of space would rapidly contract and finally disappear. Therefore, the cosmological evolution removes regions that correspond to unstable EW vacua, leaving the vast majority of universes crowded around the critical boundary. It is amusing to note the strict analogy with the case of sand dunes. The "wind" of the multiverse pushes the Higgs quartic coupling towards smaller values until space collapses under the effect of AdS gravity. As a result, the typical Higgs quartic coupling lies around the critical value.

We can also imagine alternative scenarios. Suppose that the Higgs quartic coupling is a function of some new fields $\Phi$ participating in Planckian dynamics and that their vacuum structure prefers low values of $\lambda$, as before. Once $\lambda$ becomes smaller than the critical value, the Higgs potential develops an instability at large field values. If tunnelling is sufficiently fast, the Higgs field slides towards Planckian scales. Such large Higgs configurations will in general affect the scalar potential of the fields $\Phi$, which will readjust into a different vacuum structure. The new vacua will give a different probability distribution for the Higgs quartic coupling $\lambda$ and it is imaginable that now larger values of $\lambda$ are preferred. In summary: universes in the stable or metastable phases will experience pressure towards small $\lambda$; universes in the unstable phase will experience pressure towards large $\lambda$. As a result, the most probable universes lie around the critical line separating the two phases. 
We stress that these examples do not use anthropic arguments: near-criticality is achieved by cosmological selection and/or by probability distributions in the multiverse. Nevertheless, the proximity of our universe to an inhospitable phase, as shown in figure 4, could be viewed as an indication that the principle of 'living dangerously' is at work, in a way similar to the case of the cosmological constant [135]. One can assume, as before, that the probability distribution function of $\lambda\left(M_{\mathrm{Pl}}\right)$ in the multiverse is skewed towards the lowest possible values, making it more likely for our universe to live in the leftmost region of figure 4 . The anthropic boundary of EW instability limits the allowed parameter space, giving a justification of why our universe is 'living dangerously', with conditions for stability barely satisfied.

\subsection{Double criticality of Higgs and top couplings}

From figure 4 we can infer more than just criticality of the Higgs quartic coupling. Indeed, this figure shows that the measured values of the Higgs and top masses lie in the region corresponding not only to the lowest possible values of $\lambda\left(M_{\mathrm{Pl}}\right)$ allowed by (meta)stability, but also to the smallest possible value of $y_{t}\left(M_{\mathrm{Pl}}\right)$, once $\lambda\left(M_{\mathrm{Pl}}\right)$ has been selected. Indeed, for small Higgs quartic $\left(\lambda\left(M_{\mathrm{Pl}}\right)<0.02\right)$, there is a non-vanishing minimum value of $y_{t}\left(M_{\mathrm{Pl}}\right)$ required to avoid instability.

This special feature is related to the approximate vanishing of $\beta_{\lambda}$ around the Planck mass. Indeed, for fixed $\lambda\left(M_{\mathrm{Pl}}\right)$, the top Yukawa coupling has the effect to stabilise the potential, as we evolve from high to low energies. Without a sizeable contribution from $y_{t}$, the gauge couplings tend to push $\lambda$ towards more smaller (and eventually negative) values, leading to an instability. Therefore, whenever $\lambda\left(M_{\mathrm{Pl}}\right)$ is small or negative, a nonzero $y_{t}\left(M_{\mathrm{Pl}}\right)$ is necessary to compensate the destabilising effect of gauge couplings. These considerations assume that $\lambda\left(M_{\mathrm{Pl}}\right)$ and $y_{t}\left(M_{\mathrm{Pl}}\right)$ scan widely in the multiverse, while gauge couplings do not. The case of scanning gauge couplings will be discussed in section 6.1.

It is a remarkable coincidence that the measured values of the Higgs and top masses correspond rather precisely to the simultaneous minima of both $\lambda\left(M_{\mathrm{Pl}}\right)$ and $y_{t}\left(M_{\mathrm{Pl}}\right)$. In other words, it is curious that not only do we live in the narrow vertical yellow stripe of figure 4 - the minimum of $\lambda\left(M_{\mathrm{Pl}}\right)$ - but also near the bottom of the funnel - the minimum of $y_{t}\left(M_{\mathrm{Pl}}\right)$. Near-criticality holds for both the Higgs quartic and the top Yukawa coupling. Our universe is doubly enjoying a 'dangerous life' with respect to EW stability.

\subsection{Statistics}

We can also envisage a different situation within the multiverse hypothesis, namely that $\lambda\left(M_{\mathrm{Pl}}\right)$ and $y_{t}\left(M_{\mathrm{Pl}}\right)$ are determined statistically, while neither criticality nor anthropic arguments play any role. To illustrate this possibility we argue that some of the features of the high-energy SM couplings described in section 4.1 can be explained, at a purely qualitative level, by the existence of a multiverse in which SM coupling constants scan. We will not try to address the hierarchies in the Yukawa couplings. These could emerge as the result of an underlying flavour symmetry, remnant of a sector external to the SM, although it is not excluded that the pattern of Yukawa couplings is the result of the statistical 
properties of the multiverse [136-139]. Here we keep an agnostic point of view on the issue of flavour and concentrate only on Higgs quartic, top-Yukawa, and gauge couplings.

In order to describe the multiverse, we introduce some new scalar fields $\Phi_{i}$ $(i=1, \ldots, N)$, each having $p$ different vacuum configurations. The total number of possible vacua is $p^{N}$, which is huge for large $N$. If this multiverse of vacua is a viable candidate to solve the cosmological constant problem (i.e. to explain why somewhere in the multiverse the cosmological constant could be $10^{120}$ times smaller than $M_{\mathrm{Pl}}^{4}$ ), then it is reasonable that $p^{N}$ should be at least $10^{120}$. So we envisage a situation in which $N$ is at least $\mathcal{O}\left(10^{2}\right)$, which is not inconceivable in a string framework.

To describe the scanning of the SM couplings within this multiverse, we assume that the SM fields are coupled to the fields $\Phi$ in the most general way,

$\mathcal{L}=-\frac{Z_{G}(\Phi)}{4} F_{\mu \nu} F^{\mu \nu}+Z_{H}(\Phi)\left|D_{\mu} H\right|^{2}+\left(i Z_{\psi}(\Phi) \bar{\psi} \not D \psi+Y_{a b}(\Phi) \bar{\psi}_{a} H \psi_{b}+\right.$ h.c. $)-\Lambda(\Phi)|H|^{4}$.

Here $F_{\mu \nu}$ and $\psi$ collectively denote the SM gauge and fermion fields, and $H$ is the Higgs doublet. The physical SM coupling constants are given by

$$
g=Z_{G}^{-1 / 2}, \quad y_{t}=Z_{t_{L}}^{-1 / 2} Y_{t} Z_{H}^{-1 / 2} Z_{t_{R}}^{-1 / 2}, \quad \lambda=Z_{H}^{-2} \Lambda,
$$

where the functions $Z_{G, \psi, H}, Y$, and $\Lambda$ are evaluated at a vacuum of the fields $\Phi$. Since the fields $\Phi$ have $p^{N}$ vacua, the SM couplings effectively scan in this multiverse. The coupling constants in eq. (5.2) are evaluated at the high-energy scale, here identified with $M_{\mathrm{Pl}}$, where the new dynamics is integrated out.

For simplicity, we consider the toy example of multiverse proposed in ref. [140], in which each field $\Phi_{i}$ has two vacua $(p=2)$ called $\Phi_{i}^{(+)}$and $\Phi_{i}^{(-)}$. We also assume that each of the functions $Z_{G, \psi, H}, Y, \Lambda$ (let us call them collectively $Z$, to simplify notation) can be split as a sum of the contributions of the different fields $\Phi_{i}$,

$$
Z\left(\Phi_{1}, \ldots, \Phi_{n}\right)=\sum_{i=1}^{N} Z_{i}\left(\Phi_{i}\right), \quad Z=\left\{Z_{G}, Z_{\psi}, Z_{H}, Y, \Lambda\right\}
$$

This is a consistent hypothesis, as long as the fields $\Phi$ are mutually weakly-interacting. In this case, any mixed interaction is generated only by small loop effects and can be ignored. Under this hypothesis, the $2^{N}$ values of $Z$ corresponding to the vacua of $\Phi$ can written as

$$
Z=\sum_{i=1}^{N}\left(Z_{i}^{(S)}+\eta_{i} Z_{i}^{(D)}\right), \quad Z_{i}^{(S)}=\frac{Z_{i}\left(\Phi_{i}^{(+)}\right)+Z_{i}\left(\Phi_{i}^{(-)}\right)}{2}, \quad Z_{i}^{(D)}=\frac{Z_{i}\left(\Phi_{i}^{(+)}\right)-Z_{i}\left(\Phi_{i}^{(-)}\right)}{2}
$$

where $\eta_{i}= \pm 1$. Each of the $2^{N}$ vacua (and each of the $2^{N}$ values of $Z$ ) is labeled by the vector $\eta=\left(\eta_{i}, \ldots, \eta_{N}\right)$.

The normalised probability distribution of $Z$ within the multiverse of vacua is given by

$$
\rho(Z)=2^{-N} \sum_{\eta} \delta\left(Z-N \bar{Z}-\sum_{i=1}^{N} \eta_{i} Z_{i}^{(D)}\right), \quad \bar{Z} \equiv \frac{1}{N} \sum_{i=1}^{N} Z_{i}^{(S)} .
$$


Using the central limit theorem, the discrete sum over the $2^{N}$ configurations of $\eta$ in eq. (5.5) can be approximated for large $N$ with a Gaussian distribution [140]

$$
\rho(Z)=\frac{1}{\sqrt{2 \pi N \Delta^{2}}} \exp \left[-\frac{(Z-N \bar{Z})^{2}}{2 N \Delta^{2}}\right], \quad \Delta^{2}=\frac{1}{N} \sum_{i=1}^{N} Z_{i}^{(D)^{2}} .
$$

This shows that $Z$ densely scans around $N \bar{Z}$ with an approximately flat distribution in the range $|Z-N \bar{Z}|<\sqrt{N} \Delta$.

For generic couplings, we expect that $\bar{Z}$ and $\Delta$ are quantities of order unity, and thus $Z$ is $\mathcal{O}(N)$ with a relative uncertainty of order $1 / \sqrt{N}$. Plugging this result (which is valid for $Z=Z_{G, \psi, H}, Y, \Lambda$ ) into eq. (5.2), we find

$$
g, y_{t} \sim \frac{1}{\sqrt{N}}, \quad \lambda \sim \frac{1}{N} .
$$

For $N \sim 100$, we obtain that gauge and top-Yukawa couplings are predicted to be $\mathcal{O}\left(10^{-1}\right)$ at around $M_{\mathrm{Pl}}$, while the Higgs quartic coupling is $\mathcal{O}\left(10^{-2}\right)$, in good qualitative agreement with experimental data. Indeed, adopting a 'physical' normalisation of couplings as in figure 2 (lower left), the SM predicts $g_{1,2,3}\left(M_{\mathrm{Pl}}\right) / \sqrt{2} \approx y_{t}\left(M_{\mathrm{Pl}}\right) \approx \sqrt{4\left|\lambda\left(M_{\mathrm{Pl}}\right)\right|} \approx 0.3$.

The different behaviour with $N$ in eq. (5.7) arises because $\lambda$ is a quartic coupling, while $g$ and $y_{t}$ are cubic couplings. Note that this framework suggests a hierarchy between $g, y_{t}$ on one side, and $\lambda$ on the other side, but does not predict that $\lambda$ should vanish at $M_{\mathrm{Pl}}$, again as indicated by data. Actually, since $\lambda$ scans by a relative amount $\mathcal{O}(1 / \sqrt{N})$, a vanishing value of $\lambda\left(M_{\mathrm{Pl}}\right)$ turns out to be fairly improbable in this setup.

\section{More on SM phase diagrams}

\subsection{The SM phase diagram in terms of gauge couplings}

So far we have been studying the phase diagram in terms of Higgs and top masses or couplings, keeping the other SM parameters fixed. This is reasonable, since the EW vacuum is mostly influenced by the Higgs and top quark. However, in the multiverse, other parameters can scan too and it is interesting to study how they affect our results.

We start by considering the scanning of weak couplings defined at some high-energy scale, which we identify with $M_{\mathrm{Pl}}$. The impact of the gauge couplings $g_{1}$ and $g_{2}$ can be understood from the leading terms of the RG equation for the Higgs quartic coupling

$$
(4 \pi)^{2} \frac{d \lambda}{d \ln \bar{\mu}^{2}}=-3 y_{t}^{4}+6 y_{t}^{2} \lambda+12 \lambda^{2}+\frac{9}{16}\left(g_{2}^{4}+\frac{2}{5} g_{2}^{2} g_{1}^{2}+\frac{3}{25} g_{1}^{4}\right)-\frac{9}{2} \lambda\left(g_{2}^{2}+\frac{g_{1}^{2}}{5}\right)+\cdots
$$

For small $\lambda\left(M_{\mathrm{Pl}}\right)$, the weak gauge couplings have the effect of reducing even further the Higgs quartic coupling in its evolution towards lower energies, thus contributing to destabilise the potential. For large $\lambda\left(M_{\mathrm{Pl}}\right)$, they tend to make $\lambda$ grow at lower energy.

We quantify the situation by plotting in figure 5 (left) the SM phase diagram in terms of $\lambda\left(M_{\mathrm{Pl}}\right)$ and $g_{2}\left(M_{\mathrm{Pl}}\right)$. For simplicity, we scan over the hypercharge coupling $g_{1}\left(M_{\mathrm{Pl}}\right)$ by keeping fixed the ratio $g_{1}\left(M_{\mathrm{Pl}}\right) / g_{2}\left(M_{\mathrm{Pl}}\right)=1.22$ as in the $\mathrm{SM}$, while $y_{t}\left(M_{\mathrm{Pl}}\right)$ and $g_{3}\left(M_{\mathrm{Pl}}\right)$ 

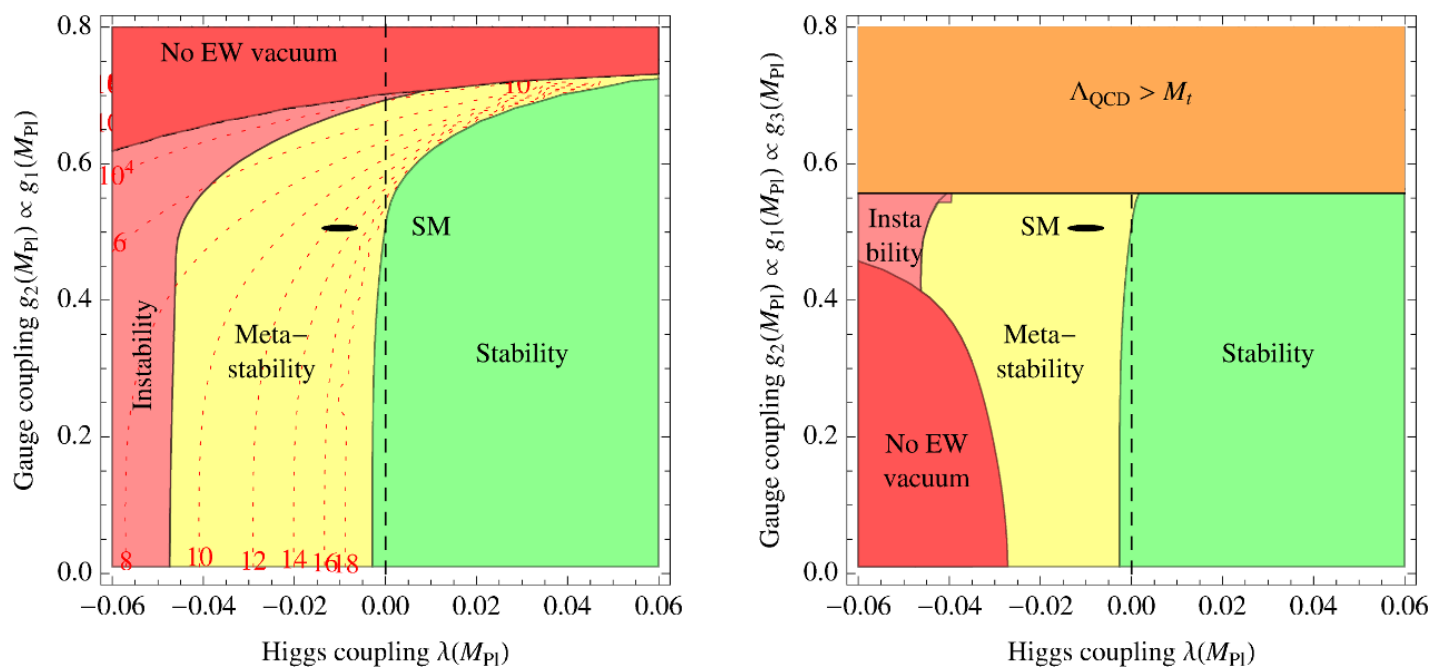

Figure 5. SM phase diagram in terms of the Higgs quartic coupling $\lambda\left(M_{\mathrm{Pl}}\right)$ and of the gauge coupling $g_{2}\left(M_{\mathrm{Pl}}\right)$. Left: a common rescaling factor is applied to the electro-weak gauge couplings $g_{1}$ and $g_{2}$, while $g_{3}$ is kept constant. Right: a common rescaling factor is applied to all SM gauge couplings $g_{1}, g_{2}, g_{3}$, such that a $10 \%$ increase in the strong gauge coupling at the Planck scale makes $\Lambda_{\mathrm{QCD}}$ larger than the weak scale. The measured values of the couplings correspond to the small ellipse marked as 'SM'.

are held to their SM values. As in previous cases, also the phase diagram in terms of weak gauge couplings shows the peculiar characteristic of the SM parameters to live close to the phase boundary. (Note that the figure is zoomed around the region of the physical values, so that the proximity to the boundary is not emphasised.)

Figure 5 (left) shows that the weak gauge couplings in the SM lie near the maximum possible values that do not lead to a premature decay of the EW vacuum. Were $g_{2}$ and $g_{1}$ $50 \%$ larger than their actual values, we wouldn't be here speculating on the peculiarity of the Higgs mass.

Next, we discuss the impact of scanning the strong gauge coupling constant. In figure 5 (right) we show the phase diagram in the plane $\lambda\left(M_{\mathrm{Pl}}\right), g_{2}\left(M_{\mathrm{Pl}}\right)$, obtained by varying all three gauge couplings by a common rescaling factor. The top Yukawa coupling $y_{t}\left(M_{\mathrm{Pl}}\right)$ is held fixed at its $\mathrm{SM}$ value and so, as the other couplings scan, the top mass does not correspond to the measured value.

The coupling $g_{3}$ affects $\beta_{\lambda}$ only at two loops, but it has a more important role in the RG evolution of the top Yukawa coupling, whose leading terms are given by

$$
(4 \pi)^{2} \frac{d y_{t}^{2}}{d \ln \bar{\mu}^{2}}=y_{t}^{2}\left(\frac{9}{2} y_{t}^{2}-8 g_{3}^{2}-\frac{9}{4} g_{2}^{2}-\frac{17}{20} g_{1}^{2}\right)+\cdots .
$$

When the value of $g_{3}$ is reduced at fixed $y_{t}\left(M_{\mathrm{Pl}}\right)$, the low-energy top Yukawa coupling becomes smaller. This reduces the stabilising effect of the top for a given $\lambda\left(M_{\mathrm{Pl}}\right)$ and explains the appearance in figure 5 (right) at small gauge couplings of a 'No EW vacuum' region (where $\lambda$ is negative at the weak scale). 


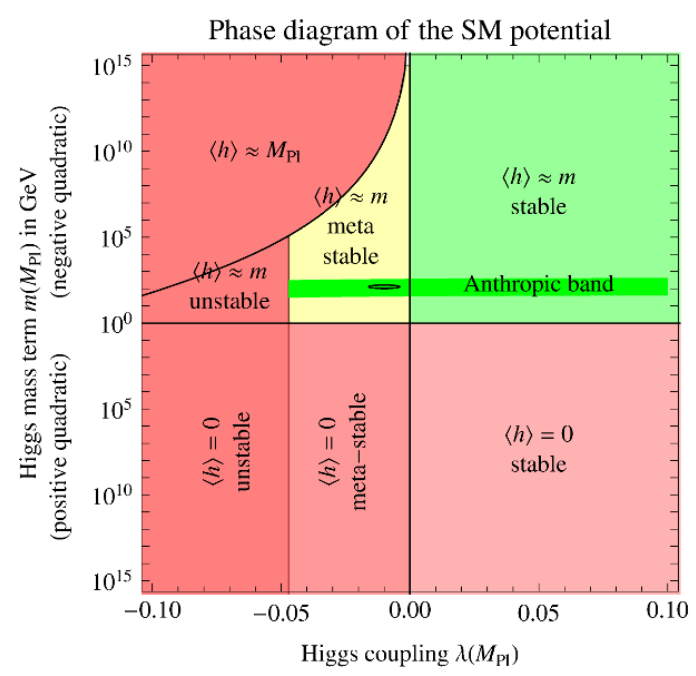

Figure 6. Phase diagram of the SM in terms of the parameters of the Higgs potential evaluated at the Planck scale. In the metastability region, there is an upper bound on $m$ from the requirement of a Higgs vacuum at a finite field value. The green region is simple thanks to the fact that $\beta(\lambda)=0$ at $M_{\mathrm{Pl}}$. On the vertical axis we plot $\left|m\left(M_{\mathrm{Pl}}\right)\right|$, in the case of negative (above) and positive (below) Higgs quadratic term.

On the other hand, when $g_{3}$ is increased, the value of $\Lambda_{\mathrm{QCD}}$ grows rapidly. Whenever

$$
\alpha_{3}\left(M_{\mathrm{Pl}}\right)>\frac{6 \pi}{21 \ln \left(M_{\mathrm{Pl}} / M_{t}\right)},
$$

which corresponds to $g_{3}\left(M_{\mathrm{Pl}}\right)>0.54$, the value of $\Lambda_{\mathrm{QCD}}$ becomes larger than $M_{t}$, preventing a perturbative extrapolation from the Planck to the weak scale. As shown in figure 5 (right), this region is reached as soon as the SM gauge couplings are increased by only $11 \%$. Once again, the SM gauge couplings live near the top of the range allowed by simple extrapolations of the minimal theory.

\subsection{The SM phase diagram in terms of Higgs potential parameters}

The Higgs mass parameter $m$ in the Higgs potential is the origin of the well-known naturalness problem. Here we show that the simple requirement of the existence of a non-trivial EW vacuum sets an upper bound on $m$, which is completely independent of any naturalness argument.

Let us start by considering the tree-level Higgs potential in eq. (2.6). For $m^{2}>0$ and $\lambda>0$, the potential has the usual non-trivial vacuum at $\langle h\rangle=v=m / \sqrt{2 \lambda}$. However, since $v$ is proportional to $m$ and $\lambda$ is negative above the instability scale $\Lambda_{I}$, the Higgs vacuum at finite field value no longer exists when $m^{2}$ is too large. The upper bound on $m^{2}$ can be estimated by considering the minimisation condition of the potential, including only the logarithmic running of $\lambda$, but neglecting the evolution of $m$ (which is a good 
approximation, as shown in figure 1):

$$
\left[2 \lambda(v)+\frac{\beta_{\lambda}(v)}{2}\right] v^{2}=m^{2} .
$$

For values of $v$ in the neighbourhood of $\Lambda_{I}$, we can approximate ${ }^{6} \lambda(v) \approx \beta_{\lambda}\left(\Lambda_{I}\right) \ln v / \Lambda_{I}$ and $\beta_{\lambda}(v) \approx \beta_{\lambda}\left(\Lambda_{I}\right)$. Then we see that eq. (6.4) has a solution only if

$$
m^{2}<-\beta_{\lambda}\left(\Lambda_{I}\right) e^{-3 / 2} \Lambda_{I}^{2}
$$

Note that $\beta_{\lambda}\left(\Lambda_{I}\right)$ is negative in the SM.

Figure 6 shows the SM phase diagram in terms of the parameters $\lambda\left(M_{\mathrm{Pl}}\right)$ and $m\left(M_{\mathrm{Pl}}\right)$. The sign of each one of these parameters corresponds to different phases of the theory, such that $\lambda\left(M_{\mathrm{Pl}}\right)=m\left(M_{\mathrm{Pl}}\right)=0$ is a tri-critical point.

The region denoted by ' $\langle h\rangle \approx M_{\mathrm{Pl}}$ ' corresponds to the case in which eq. (6.5) is not satisfied and there is no SM-like vacuum, while the Higgs field slides to large values. In the region of practical interest, the upper limit on $m$ is rather far from its actual physical value $m=M_{h}$, although it is much stronger than $M_{\mathrm{Pl}}$, the ultimate ultraviolet cutoff of the SM. A much more stringent bound on $m$ can be derived from anthropic considerations [141] and the corresponding band in parameter space is shown in figure 6 . We find it remarkable that the simple request of the existence of a non-trivial Higgs vacuum, without any reference to naturalness considerations, gives a bound on the Higgs bilinear parameter $m$. Unfortunately, for the physical value of $\lambda$, the actual numerical value of the upper bound is not of great practical importance.

\subsection{Lifetime of the SM vacuum}

The measured values of $M_{h}$ and $M_{t}$ indicate that the SM Higgs vacuum is not the true vacuum of the theory and that our universe is potentially unstable. The rate of quantum tunnelling out of the EW vacuum is given by the probability $d \wp / d V d t$ of nucleating a bubble of true vacuum within a space volume $d V$ and time interval $d t$ [142-144]

$$
d \wp=d t d V \Lambda_{B}^{4} e^{-S\left(\Lambda_{B}\right)} .
$$

In eq. (6.6), $S\left(\Lambda_{B}\right)$ is the action of the bounce of size $R=\Lambda_{B}^{-1}$, given by

$$
S\left(\Lambda_{B}\right)=\frac{8 \pi^{2}}{3\left|\lambda\left(\Lambda_{B}\right)\right|} .
$$

At the classical level, the Higgs theory with only quartic coupling is scale-invariant and the size of the bounce $\Lambda_{B}^{-1}$ is arbitrary. The RG flow breaks scale invariance and the tree level action gets replaced by the one-loop action, as calculated in ref. [28]. Then, $\Lambda_{B}$ is determined as the scale at which $\Lambda_{B}^{4} e^{-S\left(\Lambda_{B}\right)}$ is maximised. In practice this roughly amounts to minimising $\lambda\left(\Lambda_{B}\right)$, which corresponds to the condition $\beta_{\lambda}\left(\Lambda_{B}\right)=0$. As long as $\Lambda_{B} \ll M_{\mathrm{Pl}}$, gravitational effects are irrelevant, since corrections to the action in minimal

\footnotetext{
${ }^{6}$ In this analysis, we can safely neglect the non-logarithmic corrections to the effective potential and so we do not distinguish between $\lambda$ and $\lambda_{\text {eff }}$.
} 

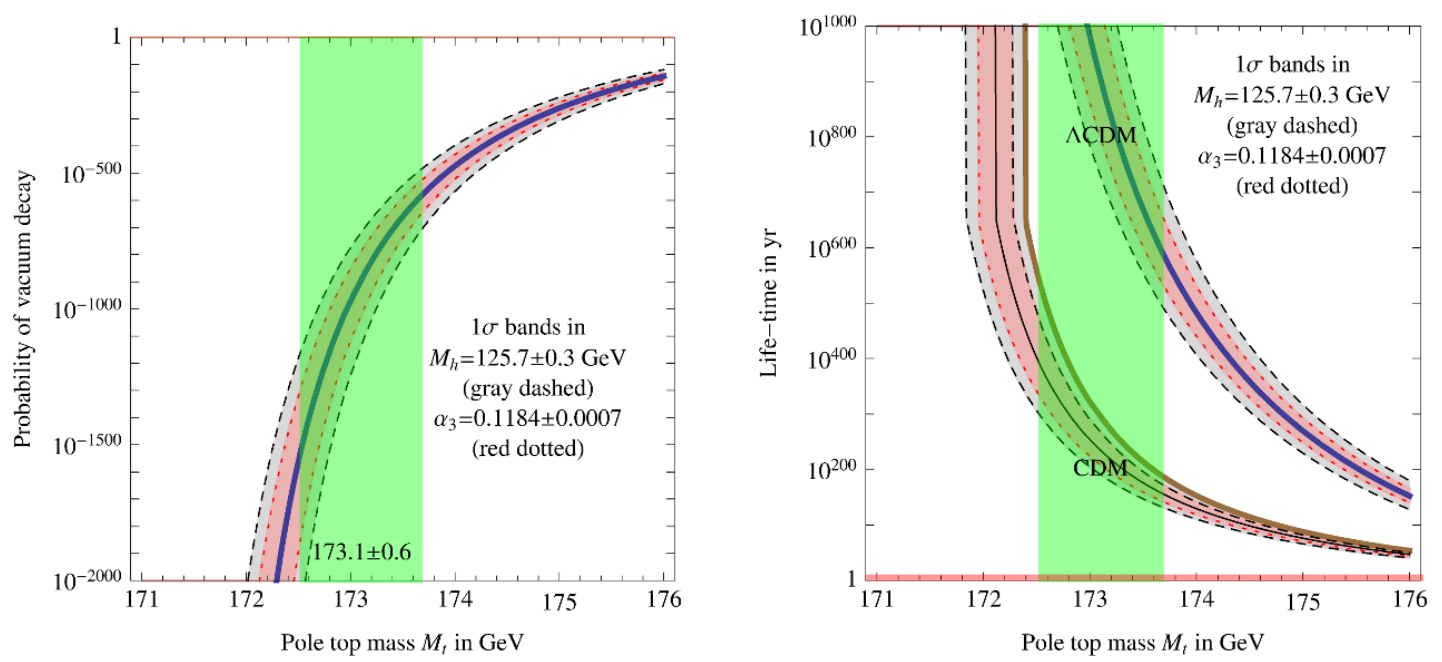

Figure 7. Left: the probability that electroweak vacuum decay happened in our past light-cone, taking into account the expansion of the universe. Right: the life-time of the electroweak vacuum, with two different assumptions for future cosmology: universes dominated by the cosmological constant $(\Lambda \mathrm{CDM})$ or by dark matter $(\mathrm{CDM})$.

Einstein gravity are given by $\delta S_{G}=256 \pi^{3} \Lambda_{B}^{2} / 45|\lambda| M_{\mathrm{Pl}}^{2}$ [119]. The effect of gravitational corrections is to slow down the tunnelling rate [145]. Whenever $\Lambda_{B}>M_{\mathrm{Pl}}$, one can only obtain a lower bound on the tunnelling probability by setting $\lambda\left(\Lambda_{B}\right)=\lambda\left(M_{\mathrm{Pl}}\right)$. In some cases, unknown Planckian dynamics can affect the tunnelling rate [57] .

The total probability $\wp$ for vacuum decay to have occurred during the history of the universe can be computed by integrating eq. (6.6) over the space-time volume of our past light-cone,

$$
\int d t d V=\int_{0}^{t_{0}} d t \int_{|x|<a\left(\eta_{0}-\eta\right)} d^{3} x=\frac{4 \pi}{3} \int_{0}^{\eta_{0}} d \eta a^{4}\left(\eta_{0}-\eta\right)^{3} \approx \frac{0.15}{H_{0}^{4}}
$$

Here $a$ is the scale factor, $\eta$ is conformal time $(d \eta / d t=1 / a), \eta_{0} \approx 3.4 / H_{0}$ is the present conformal time and $H_{0} \approx 67.4 \mathrm{~km} / \mathrm{sec} \mathrm{Mpc}$ is the present Hubble rate. Equation (6.8) roughly amounts to saying that the 'radius' of the universe is given by $c T_{U}$, where $T_{U} \approx 0.96 / H_{0}$ is the present age. The present value of the vacuum-decay probability $\wp$ is

$$
\wp_{0}=0.15 \frac{\Lambda_{B}^{4}}{H_{0}^{4}} e^{-S\left(\Lambda_{B}\right)}
$$

and is dominated by late times and this makes our result more robust, since it is independent of the early cosmological history. In figure $7 \mathrm{a}$ we plot, as a function of the top mass, the probability $\wp_{0}$ that the EW vacuum had decayed during the past history of the universe. We find that the probability is spectacularly small, as a consequence of the proximity of the SM parameters to the boundary with the region of absolute stability.

The lifetime of the present EW vacuum $\tau_{\mathrm{EW}}$ depends on the future cosmological history. If dark energy shuts off and the future universe is matter dominated, the space-time volume 
of the past light-cone at time $t_{0}$ is given by

$$
\int d t d V=\frac{4 \pi}{3} \int_{0}^{\eta_{0}} d \eta a^{4}\left(\eta_{0}-\eta\right)^{3}=\frac{16 \pi}{1485 H_{0}^{4}}
$$

Here $H_{0}$ is the Hubble parameter at time $t_{0}$, and we have performed the integral using the relations $a^{1 / 2}=H_{0} \eta / 2=\left(3 H_{0} t / 2\right)^{1 / 3}$ and $t_{0}=2 /\left(3 H_{0}\right)$, valid in a matter-dominated flat universe. The lifetime $\tau_{\mathrm{EW}}$ is given by the time at which $\wp=1$ :

$$
\tau_{\mathrm{EW}}=\left(\frac{55}{3 \pi}\right)^{1 / 4} \frac{e^{S\left(\Lambda_{B}\right) / 4}}{\Lambda_{B}} \approx \frac{T_{U}}{\wp_{0}^{1 / 4}} \quad \text { (Matter Domination) }
$$

where $\wp_{0}$ is given in eq. (6.9) and shown in figure 7a.

If instead the universe keeps being accelerated by the cosmological constant, entering into a de Sitter phase with Hubble constant $H=H_{0} \sqrt{\Omega_{\Lambda}}$, at a time $t_{0}$ in the far future the volume of the past light-cone will be

$$
\int d t d V=\frac{4 \pi}{3} \int_{0}^{\eta_{0}} d \eta a^{4}\left(\eta_{0}-\eta\right)^{3}=\frac{4 \pi}{3 H^{4}}\left[H t_{0}-\frac{11}{6}+\mathcal{O}\left(e^{-H t_{0}}\right)\right]
$$

Here we have used the relations $a=(1-H \eta)^{-1}=e^{H t}$, valid in a vacuum-energy dominated universe. The lifetime $\tau_{\mathrm{EW}}$ is now equal to

$$
\tau_{\mathrm{EW}}=\frac{3 H^{3} e^{S\left(\Lambda_{B}\right)}}{4 \pi \Lambda_{B}^{4}} \approx \frac{0.02 T_{U}}{\wp_{0}} \quad \text { (VACUUm Energy Domination). }
$$

The lifetime of the present $\mathrm{EW}$ vacuum is plotted in figure $7 \mathrm{~b}$ in both cases of matter or vacuum-energy domination. As shown, the SM vacuum is likely to survive for times that are enormously longer than any significant astrophysical age (e.g. the sun will exhaust its fuel in about five billion years).

\section{$7 \quad$ Summary and conclusions}

The measurement of the Higgs mass $M_{h}$ has determined the last unknown parameter of the SM, fixing the Higgs quartic coupling $\lambda$. Now that the experimental result is in our hands, our task as theoreticians is to interpret it, investigating whether it contains any useful information about physics at shorter distances. The first thing to try is to extrapolate $\lambda$ to high energy in search for clues. Just as high-energy extrapolations of the gauge coupling constants gave us hints about a possible grand unification of fundamental forces, so the extrapolation of $\lambda$ has revealed an unexpected feature of the SM that opens new avenues for theoretical speculation. The intriguing result is that, assuming the validity of the SM up to very high energy scales, the measured value of $M_{h}$ is near-critical, in the sense that it places the EW vacuum right at the border between absolute stability and metastability. Because of the present experimental uncertainties on the SM parameters (mostly the top quark mass), we cannot conclusively establish the fate of the EW vacuum, although metastability is now preferred at $99.3 \%$ CL. 
The special coincidence found in the value of $M_{h}$ warrants a refined calculation of the high-energy extrapolation of $\lambda$ and this was the first objective of this paper. We extracted the fundamental SM parameters $\lambda$ (quartic Higgs coupling), $m$ (Higgs mass term), $y_{t}$ (top quark Yukawa coupling), $g_{2}$ and $g_{Y}$ (electroweak gauge couplings) from the precisely measured values of the Higgs, top, $W$ and $Z$ masses and from the Fermi constant at full NNLO, by performing dedicated 2-loop computations. All couplings have been extrapolated to large energies using the RGE equations, now known at NNLO order (3 loops). We could then compute the effective potential known with 2-loop accuracy.

The second objective of this paper was to investigate the significance of the measured value of $M_{h}$, in view of its high-energy extrapolation. A first observation is that $\lambda$, together with all other SM coupling constants, remains perturbative in the entire energy domain between the Fermi and the Planck scales. This gives an indirect indication that EW-breaking dynamics is probably weakly interacting. Of course, strongly-interacting dynamics is not excluded, but there is simply no need for introducing it at any intermediate energy scale.

The most important observation concerns the stability of the Higgs potential. The critical condition for stability is defined as the vanishing of the effective coupling $\lambda_{\text {eff }}$, see eq. (4.3), at some energy scale $\Lambda_{I}$. We find $\Lambda_{I}=10^{10}-10^{12} \mathrm{GeV}$, see eq. (4.7), suggesting that the instability is reached well below the Planck mass. The presence of an instability at an intermediate scale could be interpreted as a sign of a new-physics threshold around $\Lambda_{I}$. It is suggestive that neutrino masses, axion, and inflation give independent indications for new dynamics at roughly similar energy scales. The hypothetical new physics could be responsible for a matching condition $\lambda \approx 0$ at a scale near $\Lambda_{I}$. The vanishing of $\lambda$ could be the result of special dynamics occurring above $\Lambda_{I}$, such that the evolution of the Higgs quartic is power-law suppressed, or the result of symmetry, as in the case of an approximate Goldstone boson. One of the most appealing explanations of $\lambda \approx 0$ is offered by supersymmetry, since flat directions provide a valid justification of vanishing quartic couplings for scalar particles that have other kinds of interactions at zero momentum. In this way, supersymmetry convincingly evades the problem, encountered by Goldstone bosons, of explaining why $\lambda \approx 0$ is compatible with sizeable gauge and Yukawa couplings of the Higgs boson. The scheme can be automatically realised in $N=2$ supersymmetry, while a dynamical vacuum alignment with $\tan \beta \approx 1$ is required in the case of $N=1$ supersymmetry.

Another peculiarity found in the extrapolation of $\lambda$ is its slow running at high energy. This is due to a combination of two factors: the reduction of all SM couplings at high energy and an accidental zero of $\beta_{\lambda}$ at a scale of about $10^{17}-10^{18} \mathrm{GeV}$. It is the slow running of $\lambda$ at high energy that saves the EW vacuum from premature collapse, in a situation where $\Lambda_{I} \ll M_{\mathrm{Pl}}$. Were $\beta_{\lambda}$ large and negative above $\Lambda_{I}$, we could not live with an instability scale much smaller than the cutoff scale, without being confronted with early vacuum decay. Unfortunately, for the moment we have no way to tell whether this special condition allowing for a prolonged vacuum lifetime is just a numerical coincidence or an important feature of the SM.

At any rate, the smallness of $\beta_{\lambda}$ at high energy makes it possible to assume that there is no new-physics threshold around $\Lambda_{I}$ and that the SM continues to be valid up to the quantum-gravity scale, since the tunnelling probability remains small. In this context, the 
value of $\lambda\left(M_{\mathrm{Pl}}\right)$ may be regarded as 'normal' for a SM coupling. Indeed, as discussed in section 4.1 , the ratios $\sqrt{4|\lambda|} / y_{t}$ and $\sqrt{8|\lambda|} / g_{2}$ (which, at low energy, correspond to $M_{h} / M_{t}$ and $M_{h} / M_{W}$, respectively) are of order unity both at the Fermi and Planck scales. The vanishing of $\lambda$ at an intermediate scale could then be purely accidental. After all, the Higgs quartic is the only SM coupling that can cross zero during its RG evolution, since $\lambda=0$ is not a point of enhanced symmetry.

In our view, the most interesting aspect of the measured value of $M_{h}$ is its nearcriticality. In this paper we have thoroughly studied the condition of near-criticality in terms of the SM parameters at a high scale, which we identified with the Planck mass. This procedure is more appropriate than a study in terms of physical particle masses, since it is more likely that special features are exhibited by high-energy parameters, just like in the case of gauge coupling unification.

We have found that near-criticality is manifest also when we explore the phase diagram as a function of high-energy SM couplings. Moreover, we found evidence for multiple near-critical conditions. Indeed, the measured SM parameters roughly correspond to the minimum values of Higgs quartic coupling $\lambda\left(M_{\mathrm{Pl}}\right)$ and of the top Yukawa coupling $y_{t}\left(M_{\mathrm{Pl}}\right)$ (at fixed gauge couplings) that allow for the existence of a sufficiently long-lived EW vacuum. Moreover, at fixed top Yukawa coupling, the maximum possible values of the gauge couplings $g\left(M_{\mathrm{Pl}}\right)$ are preferred. Incidentally, we have also obtained an upper bound on the Higgs mass parameter $m$ from the requirement of vacuum stability, although this bound is too weak to be useful in practice.

We explored possible interpretations of this multiple near-criticality. Provided it is not just a fortuitous coincidence, an explanation of near-criticality almost necessarily requires the existence of an underlying statistical system. This drives us towards the multiverse as the most convincing framework in which one can address the issue. Near-criticality can emerge in the multiverse from an appropriate probabilistic pressure in the space of coupling constants, together with the anthropic requirement that selects universes in which the life-friendly EW vacuum is sufficiently long-lived. The principle of 'living dangerously' populates universes close to the boundary of a hospitable phase, just as it is conjectured to happen in the case of the cosmological constant.

In this context, one may wonder whether the LHC measurement of the Higgs mass corresponds to a point in parameter space that is sufficiently close to the instability boundary to be justified by the principle of 'living dangerously'. Unfortunately, the answer to this question depends on the unknown probability distribution of the SM couplings that scan. In the case of the cosmological constant, we have a clear understanding of why larger values of $\Lambda_{\mathrm{CC}}$ should be preferred within the universe: small values of $\Lambda_{\mathrm{CC}}$ require delicate accidental cancellations among the various parameters of the theory. On the other hand, here we are dealing with dimensionless couplings and it is less clear why there should be any probabilistic preference and, especially, in which direction should the multiverse pressure act. It is plausible that renormalisable couplings, such as $\lambda$, have a less steep probability distribution than the cosmological constant and therefore are likely to show a less pronounced proximity to the critical boundary in a given individual universe, but of course it is impossible to make definitive statements at this stage. 
It is interesting that near-criticality could find an explanation in the multiverse, without any reference to anthropic reasoning. In nature there exist statistical systems in which criticality is an attractor point of their dynamical evolution. If such a phenomenon took place in the multiverse, then the majority of universes would populate regions close to phase transitions. Such a (non-anthropic) explanation of near-criticality of the Higgs mass could also provide a link to the naturalness problem, since the smallness of the mass parameter $m$ in the Higgs potential is near-critical with respect to the EW symmetry-breaking phase transition. It is indeed a remarkable experimental fact that both $\lambda$ and $m$ (the two parameters of the Higgs potential) happen to lie very close to boundaries between different phases of the SM. So, according to this interpretation, our universe would not be a rare occurrence in the multiverse where SM parameters are selected in such a way that the cosmological evolution of the EW vacuum is favourable to life. On the contrary, near-criticality of the Higgs parameters would be a fairly generic property of the multiverse and our universe would be unexceptional.

In spite of the absence of any signal of new physics, the LHC has already provided valuable information for theoretical speculations about physics at very short distances. In that respect, the most important result has been the near-criticality of the Higgs mass the subject of this paper.

\section{Acknowledgments}

This work was supported by the SF0690030s09 project, by the Research Executive Agency (REA) of the European Union under the Grant Agreement number PITN-GA- 2010-264564 (LHCPhenoNet); by the EU ITN "Unification in the LHC Era", contract PITN-GA2009-237920 (UNILHC) and by MIUR under contract 2006022501; by the Spanish Ministry of Economy and Competitiveness under grant FPA2012-32828, Consolider-CPAN (CSD2007-00042), the grant SEV-2012-0249 of the "Centro de Excelencia Severo Ochoa" Programme and the grant HEPHACOS-S2009/ESP1473 from the C.A. de Madrid. We thank Simone Alioli, Claudio Bonati, Lawrence Hall, Luis E. Ibáñez, Gino Isidori, and Riccardo Rattazzi for useful discussions. The work of P.P.G. has been partially funded by the "Fondazione A. Della Riccia".

\section{A Weak scale thresholds at one loop}

We summarise here the one-loop corrections $\theta^{(1)}$ to the various SM parameters

$$
\theta=\left\{\lambda, m, y_{t}, g_{2}, g_{Y}\right\}=\theta^{(0)}+\theta^{(1)}+\theta^{(2)}+\cdots .
$$

We perform one-loop computations in a generic $\xi$ gauge, confirming that $\theta^{(1)}$ is gaugeindependent, as it should. Our expressions for $\theta^{(1)}$ are equivalent to the well known expressions in the literature. We write $\theta^{(1)}$ in terms of finite parts of the the Passarino-Veltman functions

$$
A_{0}(M)=M^{2}\left(1-\ln \frac{M^{2}}{\bar{\mu}^{2}}\right), \quad B_{0}\left(p ; M_{1}, M_{2}\right)=-\int_{0}^{1} \ln \frac{x M_{1}^{2}+(1-x) M_{2}^{2}-x(1-x) p^{2}}{\bar{\mu}^{2}} d x .
$$


The dependence of $\theta^{(1)}$ on the renormalisation scale $\bar{\mu}$ reproduces the well known one-loop RGE equations for $\theta$. Below we report the expressions valid in the limit $M_{b}=M_{\tau}=0$; the negligible effect of light fermions masses is included in our full code.

\section{A.1 The quartic Higgs coupling}

The one-loop result is obtained from eq. (2.13):

$$
\begin{aligned}
\lambda^{(1)}(\bar{\mu})= & \frac{1}{(4 \pi)^{2} V^{4}} \operatorname{Re}\left[3 M_{t}^{2}\left(M_{h}^{2}-4 M_{t}^{2}\right) B_{0}\left(M_{h} ; M_{t}, M_{t}\right)+3 M_{h}^{2} A_{0}\left(M_{t}\right)+\right. \\
& +\frac{1}{4}\left(M_{h}^{4}-4 M_{h}^{2} M_{Z}^{2}+12 M_{Z}^{4}\right) B_{0}\left(M_{h} ; M_{Z}, M_{Z}\right)+\frac{M_{h}^{2}\left(7 M_{W}^{2}-4 M_{Z}^{2}\right)}{2\left(M_{Z}^{2}-M_{W}^{2}\right)} A_{0}\left(M_{Z}\right)+ \\
& +\frac{1}{2}\left(M_{h}^{4}-4 M_{h}^{2} M_{W}^{2}+12 M_{W}^{4}\right) B_{0}\left(M_{h} ; M_{W}, M_{W}\right)-\frac{3 M_{h}^{2} M_{W}^{2}}{2\left(M_{h}^{2}-M_{W}^{2}\right)} A_{0}\left(M_{h}\right)+(\mathrm{A} .3) \\
& +\frac{M_{h}^{2}}{2}\left(-11+\frac{3 M_{h}^{2}}{M_{h}^{2}-M_{W}^{2}}-\frac{3 M_{W}^{2}}{M_{Z}^{2}-M_{W}^{2}}\right) A_{0}\left(M_{W}\right)+ \\
& \left.+\frac{9}{4} M_{h}^{4} B_{0}\left(M_{h} ; M_{h}, M_{h}\right)+\frac{1}{4}\left(M_{h}^{4}+M_{h}^{2}\left(M_{Z}^{2}+2 M_{W}^{2}-6 M_{t}^{2}\right)-8\left(M_{Z}^{4}+2 M_{W}^{4}\right)\right)\right] .
\end{aligned}
$$

Each one of the terms in eq. (2.13) is gauge dependent, e.g. the one-loop correction to muon decay is

$$
\begin{aligned}
\left.\Delta r_{0}^{(1)}\right|_{\text {fin }}= & \frac{1}{(4 \pi V)^{2}}\left[3 M_{t}^{2}-M_{W}^{2}-\frac{M_{Z}^{2}}{2}-\frac{M_{h}^{2}}{2}+\frac{3 M_{W}^{2} A_{0}\left(M_{h}\right)}{M_{h}^{2}-M_{W}^{2}}+\frac{6 M_{W}^{2}-3 M_{Z}^{2}}{M_{W}^{2}-M_{Z}^{2}} A_{0}\left(M_{Z}\right)+\right.\text { (A.4) } \\
& \left.-6 A_{0}\left(M_{t}\right)+\left(9-\frac{3 M_{h}^{2}}{M_{h}^{2}-M_{W}^{2}}-\frac{3 M_{W}^{2}}{M_{W}^{2}-M_{Z}^{2}}\right) A_{0}\left(M_{W}\right)+2 A_{0}\left(\sqrt{\xi} M_{W}\right)+A_{0}\left(\sqrt{\xi} M_{Z}\right)\right]
\end{aligned}
$$

and the gauge dependence cancels out in the sum $\lambda^{(1)}(\bar{\mu})$.

\section{A.2 The Higgs mass term}

The correction is obtained from eq. (2.15):

$$
\begin{aligned}
\delta^{(1)} m^{2}(\bar{\mu})= & \frac{1}{(4 \pi)^{2} V^{2}} \operatorname{Re}\left[6 M_{t}^{2}\left(M_{h}^{2}-4 M_{t}^{2}\right) B_{0}\left(M_{h} ; M_{t}, M_{t}\right)+24 M_{t}^{2} A_{0}\left(M_{t}\right)+\right. \\
& +\left(M_{h}^{4}-4 M_{h}^{2} M_{W}^{2}+12 M_{W}^{4}\right) B_{0}\left(M_{h} ; M_{W}, M_{W}\right)-2\left(M_{h}^{2}+6 M_{W}^{2}\right) A_{0}\left(M_{W}\right)+ \\
& +\frac{1}{2}\left(M_{h}^{4}-4 M_{h}^{2} M_{Z}^{2}+12 M_{Z}^{4}\right) B_{0}\left(M_{h} ; M_{Z}, M_{Z}\right)-\left(M_{h}^{2}+6 M_{Z}^{2}\right) A_{0}\left(M_{Z}\right)+ \\
& \left.+\frac{9}{2} M_{h}^{4} B_{0}\left(M_{h} ; M_{h}, M_{h}\right)-3 M_{h}^{2} A_{0}\left(M_{h}\right)\right] .
\end{aligned}
$$

\section{A.3 The top Yukawa coupling}

The gauge-invariant one-loop correction to the top Yukawa coupling is obtained from eq. (2.19)

$$
\begin{aligned}
y_{t}^{(1)}(\bar{\mu})= & \frac{M_{t}}{\sqrt{2} V^{3}(4 \pi)^{2}} \operatorname{Re}\left[-\left(M_{h}^{2}-4 M_{t}^{2}\right) B_{0}\left(M_{t} ; M_{h}, M_{t}\right)+\right. \\
& +\frac{M_{t}^{2}\left(80 M_{W}^{2} M_{Z}^{2}-64 M_{W}^{4}-7 M_{Z}^{4}\right)+40 M_{W}^{2} M_{Z}^{4}-32 M_{W}^{4} M_{Z}^{2}-17 M_{Z}^{6}}{9 M_{t}^{2} M_{Z}^{2}} B_{0}\left(M_{t} ; M_{t}, M_{Z}\right)+
\end{aligned}
$$




$$
\begin{aligned}
& +\frac{\left(M_{t}^{2} M_{W}^{2}+M_{t}^{4}-2 M_{W}^{4}\right)}{M_{t}^{2}} B_{0}\left(M_{t} ; 0, M_{W}\right)+ \\
& +\left(\frac{3 M_{h}^{2}}{M_{h}^{2}-M_{W}^{2}}+\frac{2 M_{W}^{2}}{M_{t}^{2}}+\frac{3 M_{W}^{2}}{M_{W}^{2}-M_{Z}^{2}}-10\right) A_{0}\left(M_{W}\right)+\left(\frac{3 M_{W}^{2}}{M_{W}^{2}-M_{h}^{2}}+1\right) A_{0}\left(M_{h}\right)+ \\
& +\frac{\left(36 M_{t}^{2} M_{Z}^{2}-56 M_{W}^{2} M_{Z}^{2}+64 M_{W}^{4}-17 M_{Z}^{4}\right)}{9 M_{t}^{2} M_{Z}^{2}} A_{0}\left(M_{t}\right)+ \\
& +\left(\frac{3 M_{W}^{2}}{M_{Z}^{2}-M_{W}^{2}}+\frac{32 M_{W}^{4}-40 M_{W}^{2} M_{Z}^{2}+17 M_{Z}^{4}}{9 M_{t}^{2} M_{Z}^{2}}-3\right) A_{0}\left(M_{Z}\right)+ \\
& \left.+\frac{M_{h}^{2}}{2}-3 M_{t}^{2}-9 M_{W}^{2}+\frac{7 M_{Z}^{2}}{18}+\frac{64 M_{W}^{2}}{9 M_{Z}^{2}}\right]+\frac{M_{t}}{\sqrt{2} V(4 \pi)^{2}} g_{3}^{2}\left(-\frac{8 A_{0}\left(M_{t}\right)}{M_{t}^{2}}-\frac{8}{3}\right)
\end{aligned}
$$

\section{A.4 The weak gauge couplings}

The one-loop correction to the $\mathrm{SU}(2)_{L}$ gauge coupling is obtained from eq. (2.21):

$$
\begin{aligned}
g_{2}^{(1)}(\bar{\mu})= & \frac{2 M_{W}}{(4 \pi)^{2} V^{3}} \operatorname{Re}\left[\left(\frac{M_{h}^{4}}{6 M_{W}^{2}}-\frac{2 M_{h}^{2}}{3}+2 M_{W}^{2}\right) B_{0}\left(M_{W}, M_{h}, M_{W}\right)+\right. \\
& +\left(-\frac{M_{t}^{4}}{M_{W}^{2}}-M_{t}^{2}+2 M_{W}^{2}\right) B_{0}\left(M_{W}, 0, M_{t}\right)+ \\
& +\frac{1}{6}\left(-\frac{48 M_{W}^{4}}{M_{Z}^{2}}+\frac{M_{Z}^{4}}{M_{W}^{2}}-68 M_{W}^{2}+16 M_{Z}^{2}\right) B_{0}\left(M_{W}, M_{W}, M_{Z}\right)+ \\
& +\frac{1}{6}\left(M_{h}^{2}\left(\frac{9}{M_{h}^{2}-M_{W}^{2}}+\frac{1}{M_{W}^{2}}\right)+\frac{M_{Z}^{2}}{M_{W}^{2}}+M_{W}^{2}\left(\frac{9}{M_{W}^{2}-M_{Z}^{2}}+\frac{48}{M_{Z}^{2}}\right)-27\right) A_{0}\left(M_{W}\right)+ \\
& +\left(2-\frac{M_{h}^{2}\left(M_{h}^{2}+8 M_{W}^{2}\right)}{6 M_{W}^{2}\left(M_{h}^{2}-M_{W}^{2}\right)}\right) A_{0}\left(M_{h}\right)+\left(\frac{M_{t}^{2}}{M_{W}^{2}}+1\right) A_{0}\left(M_{t}\right)+ \\
& +\frac{1}{6}\left(\frac{24 M_{W}^{2}}{M_{Z}^{2}}-\frac{M_{Z}^{2}}{M_{W}^{2}}+\frac{9 M_{W}^{2}}{M_{Z}^{2}-M_{W}^{2}}-17\right) A_{0}\left(M_{Z}\right)+ \\
& \left.+\frac{1}{36}\left(-3 M_{h}^{2}+18 M_{t}^{2}+\frac{288 M_{W}^{4}}{M_{Z}^{2}}-374 M_{W}^{2}-3 M_{Z}^{2}\right)\right]
\end{aligned}
$$

The one-loop correction to the $\mathrm{U}(1)_{\mathrm{Y}}$ gauge coupling is obtained from eq. (2.23):

$$
\begin{aligned}
g_{Y}^{(1)}(\bar{\mu})= & \frac{2 \sqrt{M_{Z}^{2}-M_{W}^{2}}}{(4 \pi)^{2} V^{3}} \operatorname{Re}\left[\left(\frac{88}{9}-\frac{124 M_{W}^{2}}{9 M_{Z}^{2}}+\frac{M_{h}^{2}+34 M_{W}^{2}}{6\left(M_{Z}^{2}-M_{W}^{2}\right)}\right) A_{0}\left(M_{Z}\right)+\right. \\
& +\frac{M_{h}^{2}-4 M_{W}^{2}}{2\left(M_{h}^{2}-M_{W}^{2}\right)} A_{0}\left(M_{h}\right)+\left(-\frac{7}{9}-\frac{M_{t}^{2}}{M_{Z}^{2}-M_{W}^{2}}+\frac{64 M_{W}^{2}}{9 M_{Z}^{2}}\right) A_{0}\left(M_{t}\right)+ \\
& +\frac{M_{h}^{4}+2 M_{W}^{2}\left(M_{W}^{2}-15 M_{Z}^{2}\right)+3 M_{H}^{2}\left(2 M_{W}^{2}+7 M_{Z}^{2}\right)}{6\left(M_{h}^{2}-M_{W}^{2}\right)\left(M_{W}^{2}-M_{Z}^{2}\right)} A_{0}\left(M_{W}\right)+ \\
& -\frac{M_{t}^{4}+M_{W}^{2} M_{t}^{2}-2 M_{W}^{4}}{M_{W}^{2}-M_{Z}^{2}} B_{0}\left(M_{W}, 0, M_{t}\right)-\frac{M_{h}^{4}-4 M_{Z}^{2} M_{h}^{2}+12 M_{Z}^{4}}{6\left(M_{W}^{2}-M_{Z}^{2}\right)}\left(M_{Z}, M_{h}, M_{Z}\right)+ \\
& +\frac{M_{h}^{4}-4 M_{W}^{2} M_{h}^{2}+12 M_{W}^{4}}{6\left(M_{W}^{2}-M_{Z}^{2}\right)} B_{0}\left(M_{W}, M_{h}, M_{W}\right)+ \\
& +\frac{M_{Z}^{6}-48 M_{W}^{6}-68 M_{Z}^{2} M_{W}^{4}+16 M_{Z}^{4} M_{W}^{2}}{6 M_{Z}^{2}\left(M_{W}^{2}-M_{Z}^{2}\right)} \\
& +\frac{1}{9}\left(-23 M_{W}^{2}+7 M_{t}^{2}+17 M_{Z}^{2}-\frac{64 M_{t}^{2} M_{W}^{2}}{M_{Z}^{2}}-\frac{9 M_{W}^{2}\left(M_{t}^{2}-M_{W}^{2}\right)}{M_{Z}^{2}-M_{W}^{2}}\right) B_{0}\left(M_{Z}, M_{t}, M_{t}\right)+
\end{aligned}
$$




$$
\begin{aligned}
& +\frac{M_{Z}^{6}-48 M_{W}^{6}-68 M_{Z}^{2} M_{W}^{4}+16 M_{Z}^{4} M_{W}^{2}}{6 M_{Z}^{2}\left(M_{Z}^{2}-M_{W}^{2}\right)} B_{0}\left(M_{Z}, M_{W}, M_{W}\right)+ \\
& \left.+\frac{1}{36}\left(\frac{576 M_{W}^{4}}{M_{Z}^{2}}-242 M_{W}^{2}-3 M_{h}^{2}+257 M_{Z}^{2}+\frac{36 M_{W}^{2}}{M_{Z}^{2}-M_{W}^{2}}+M_{t}^{2}\left(82-\frac{256 M_{W}^{2}}{M_{Z}^{2}}\right)\right)\right]
\end{aligned}
$$

\section{B SM RGE equations up to three loops}

We list here the known results for the renormalisation group equations up to 3 loop order for the sizeable SM couplings, $g_{1}, g_{2}, g_{3}, y_{t}$ and $\lambda$ in the $\overline{\mathrm{MS}}$ scheme. We write numerically those 3 -loop coefficients that involve the $\zeta_{3}$ constant. Stopping for simplicity at two loops, we also write RGE equations for the smaller bottom and tau Yukawa coupling and their contributions to the RGE of the large couplings. Our numerical code includes full RGE at 3 loops.

\section{B.1 Gauge couplings}

RGE for the hypercharge gauge coupling in GUT normalisation $\left(g_{1}^{2}=5 g_{Y}^{2} / 3\right)$ :

$$
\begin{aligned}
\frac{d g_{1}^{2}}{d \ln \bar{\mu}^{2}}= & \frac{g_{1}^{4}}{(4 \pi)^{2}}\left[\frac{41}{10}\right]+\frac{g_{1}^{4}}{(4 \pi)^{4}}\left[\frac{44 g_{3}^{2}}{5}+\frac{27 g_{2}^{2}}{10}+\frac{199 g_{1}^{2}}{50}-\frac{17 y_{t}^{2}}{10}-\frac{y_{b}^{2}}{2}-\frac{3 y_{\tau}^{2}}{2}\right]+ \\
& +\frac{g_{1}^{4}}{(4 \pi)^{6}}\left[y_{t}^{2}\left(\frac{189 y_{t}^{2}}{16}-\frac{29 g_{3}^{2}}{5}-\frac{471 g_{2}^{2}}{32}-\frac{2827 g_{1}^{2}}{800}\right)+\lambda\left(-\frac{36 \lambda}{5}+\frac{9 g_{2}^{2}}{5}+\frac{27 g_{1}^{2}}{25}\right)+\right. \\
& \left.+\frac{297 g_{3}^{4}}{5}+\frac{789 g_{2}^{4}}{64}-\frac{388613 g_{1}^{4}}{24000}-\frac{3 g_{3}^{2} g_{2}^{2}}{5}-\frac{137 g_{3}^{2} g_{1}^{2}}{75}+\frac{123 g_{2}^{2} g_{1}^{2}}{160}\right]
\end{aligned}
$$

RGE for the $\mathrm{SU}(2)_{L}$ gauge coupling:

$$
\begin{aligned}
\frac{d g_{2}^{2}}{d \ln \bar{\mu}^{2}}= & \frac{g_{2}^{4}}{(4 \pi)^{2}}\left[-\frac{19}{6}\right]+\frac{g_{2}^{4}}{(4 \pi)^{4}}\left[12 g_{3}^{2}+\frac{35 g_{2}^{2}}{6}+\frac{9 g_{1}^{2}}{10}-\frac{3 y_{t}^{2}}{2}-\frac{3 y_{b}^{2}}{2}-\frac{y_{\tau}^{2}}{2}\right]+ \\
& +\frac{g_{2}^{4}}{(4 \pi)^{6}}\left[y_{t}^{2}\left(\frac{147 y_{t}^{2}}{16}-7 g_{3}^{2}-\frac{729 g_{2}^{2}}{32}-\frac{593 g_{1}^{2}}{160}\right)+\lambda\left(-3 \lambda+\frac{3 g_{2}^{2}}{2}+\frac{3 g_{1}^{2}}{10}\right)+\right. \\
& \left.+81 g_{3}^{4}+\frac{324953 g_{2}^{4}}{1728}-\frac{5597 g_{1}^{4}}{1600}+39 g_{3}^{2} g_{2}^{2}-\frac{g_{3}^{2} g_{1}^{2}}{5}+\frac{873 g_{2}^{2} g_{1}^{2}}{160}\right] .
\end{aligned}
$$

RGE for the strong gauge coupling, including also pure QCD terms at 4 loops:

$$
\begin{aligned}
\frac{d g_{3}^{2}}{d \ln \bar{\mu}^{2}}= & \frac{g_{3}^{4}}{(4 \pi)^{2}}[-7]+\frac{g_{3}^{4}}{(4 \pi)^{4}}\left[-26 g_{3}^{2}+\frac{9 g_{2}^{2}}{2}+\frac{11 g_{1}^{2}}{10}-2 y_{t}^{2}-2 y_{b}^{2}\right]+ \\
& +\frac{g_{3}^{4}}{(4 \pi)^{6}}\left[y_{t}^{2}\left(15 y_{t}^{2}-40 g_{3}^{2}-\frac{93 g_{2}^{2}}{8}-\frac{101 g_{1}^{2}}{40}\right)+\right. \\
& \left.+\frac{65 g_{3}^{4}}{2}+\frac{109 g_{2}^{4}}{8}-\frac{523 g_{1}^{4}}{120}+21 g_{3}^{2} g_{2}^{2}+\frac{77 g_{3}^{2} g_{1}^{2}}{15}-\frac{3 g_{2}^{2} g_{1}^{2}}{40}\right]+\frac{g_{3}^{10}}{(4 \pi)^{8}}[-2472.28] .
\end{aligned}
$$

\section{B.2 Higgs quartic coupling}

RGE for the Higgs quartic coupling:

$$
\begin{aligned}
\frac{d \lambda}{d \ln \bar{\mu}^{2}}= & \frac{1}{(4 \pi)^{2}}\left[\lambda\left(12 \lambda+6 y_{t}^{2}+6 y_{b}^{2}+2 y_{\tau}^{2}-\frac{9 g_{2}^{2}}{2}-\frac{9 g_{1}^{2}}{10}\right)-3 y_{t}^{4}-3 y_{b}^{4}-y_{\tau}^{4}+\frac{9 g_{2}^{4}}{16}+\frac{27 g_{1}^{4}}{400}+\frac{9 g_{2}^{2} g_{1}^{2}}{40}\right]+ \\
& +\frac{1}{(4 \pi)^{4}}\left[\lambda^{2}\left(-156 \lambda-72 y_{t}^{2}-72 y_{b}^{2}-24 y_{\tau}^{2}+54 g_{2}^{2}+\frac{54 g_{1}^{2}}{5}\right)+\lambda y_{t}^{2}\left(-\frac{3 y_{t}^{2}}{2}-21 y_{b}^{2}+40 g_{3}^{2}+\right.\right. \\
& \left.+\frac{45 g_{2}^{2}}{4}+\frac{17 g_{1}^{2}}{4}\right)+\lambda y_{b}^{2}\left(-\frac{3 y_{b}^{2}}{2}+40 g_{3}^{2}+\frac{45 g_{2}^{2}}{4}+\frac{5 g_{1}^{2}}{4}\right)+\lambda y_{\tau}^{2}\left(-\frac{y_{\tau}^{2}}{2}+\frac{15 g_{2}^{2}}{4}+\frac{15 g_{1}^{2}}{4}\right)+
\end{aligned}
$$




$$
\begin{aligned}
& \lambda\left(-\frac{73 g_{2}^{4}}{16}+\frac{1887 g_{1}^{4}}{400}+\frac{117 g_{2}^{2} g_{1}^{2}}{40}\right)+y_{t}^{4}\left(15 y_{t}^{2}-3 y_{b}^{2}-16 g_{3}^{2}-\frac{4 g_{1}^{2}}{5}\right)+ \\
& +y_{t}^{2}\left(-\frac{9 g_{2}^{4}}{8}-\frac{171 g_{1}^{4}}{200}+\frac{63 g_{2}^{2} g_{1}^{2}}{20}\right)+y_{b}^{4}\left(-3 y_{t}^{2}+15 y_{b}^{2}-16 g_{3}^{2}+\frac{2 g_{1}^{2}}{5}\right)+ \\
& +y_{b}^{2}\left(-\frac{9 g_{2}^{4}}{8}+\frac{9 g_{1}^{4}}{40}+\frac{27 g_{2}^{2} g_{1}^{2}}{20}\right)+y_{\tau}^{4}\left(5 y_{\tau}^{2}-\frac{6 g_{1}^{2}}{5}\right)+y_{\tau}^{2}\left(-\frac{3 g_{2}^{4}}{8}-\frac{9 g_{1}^{4}}{8}+\frac{33 g_{2}^{2} g_{1}^{2}}{20}\right)+ \\
& \left.+\frac{305 g_{2}^{6}}{32}-\frac{3411 g_{1}^{6}}{4000}-\frac{289 g_{2}^{4} g_{1}^{2}}{160}-\frac{1677 g_{2}^{2} g_{1}^{4}}{800}\right]+ \\
& +\frac{1}{(4 \pi)^{6}}\left[\lambda^{3}\left(6011.35 \lambda+873 y_{t}^{2}-387.452 g_{2}^{2}-77.490 g_{1}^{2}\right)+\lambda^{2} y_{t}^{2}\left(1768.26 y_{t}^{2}+160.77 g_{3}^{2}+\right.\right. \\
& \left.-359.539 g_{2}^{2}-63.869 g_{1}^{2}\right)+\lambda^{2}\left(-790.28 g_{2}^{4}-185.532 g_{1}^{4}-316.64 g_{2}^{2} g_{1}^{2}\right)+\lambda y_{t}^{4}\left(-223.382 y_{t}^{2}+\right. \\
& \left.-662.866 g_{3}^{2}-5.470 g_{2}^{2}-21.015 g_{1}^{2}\right)+\lambda y_{t}^{2}\left(356.968 g_{3}^{4}-319.664 g_{2}^{4}-74.8599 g_{1}^{4}+15.1443 g_{3}^{2} g_{2}^{2}+\right. \\
& \left.+17.454 g_{3}^{2} g_{1}^{2}+5.615 g_{2}^{2} g_{1}^{2}\right)+\lambda g_{2}^{4}\left(-57.144 g_{3}^{2}+865.483 g_{2}^{2}+79.638 g_{1}^{2}\right)+\lambda g_{1}^{4}\left(-8.381 g_{3}^{2}+\right. \\
& \left.+61.753 g_{2}^{2}+28.168 g_{1}^{2}\right)+y_{t}^{6}\left(-243.149 y_{t}^{2}+250.494 g_{3}^{2}+74.138 g_{2}^{2}+33.930 g_{1}^{2}\right)+ \\
& +y_{t}^{4}\left(-50.201 g_{3}^{4}+15.884 g_{2}^{4}+15.948 g_{1}^{4}+13.349 g_{3}^{2} g_{2}^{2}+17.570 g_{3}^{2} g_{1}^{2}-70.356 g_{2}^{2} g_{1}^{2}\right)+ \\
& +y_{t}^{2} g_{3}^{2}\left(16.464 g_{2}^{4}+1.016 g_{1}^{4}+11.386 g_{2}^{2} g_{1}^{2}\right)+y_{t}^{2} g_{2}^{4}\left(62.500 g_{2}^{2}+13.041 g_{1}^{2}\right)+ \\
& +y_{t}^{2} g_{1}^{4}\left(10.627 g_{2}^{2}+11.117 g_{1}^{2}\right)+g_{3}^{2}\left(7.536 g_{2}^{6}+0.663 g_{1}^{6}+1.507 g_{2}^{4} g_{1}^{2}+1.105 g_{2}^{2} g_{1}^{4}\right)+ \\
& \left.-114.091 g_{2}^{8}-1.508 g_{1}^{8}-37.889 g_{2}^{6} g_{1}^{2}+6.500 g_{2}^{4} g_{1}^{4}-1.543 g_{2}^{2} g_{1}^{6}\right] .
\end{aligned}
$$

\section{B.3 Higgs mass term}

RGE for the Higgs mass term:

$$
\begin{aligned}
\frac{d m^{2}}{d \ln \bar{\mu}^{2}}= & \frac{m^{2}}{(4 \pi)^{2}}\left[6 \lambda+3 y_{t}^{2}+3 y_{b}^{2}+y_{\tau}^{2}-\frac{9 g_{2}^{2}}{4}-\frac{9 g_{1}^{2}}{20}\right]+ \\
& +\frac{m^{2}}{(4 \pi)^{4}}\left[\lambda\left(-30 \lambda-36 y_{t}^{2}-36 y_{b}^{2}-12 y_{\tau}^{2}+36 g_{2}^{2}+\frac{36 g_{1}^{2}}{5}\right)+\right. \\
& +y_{t}^{2}\left(-\frac{27 y_{t}^{2}}{4}-\frac{21 y_{b}^{2}}{2}+20 g_{3}^{2}+\frac{45 g_{2}^{2}}{8}+\frac{17 g_{1}^{2}}{8}\right)+y_{b}^{2}\left(-\frac{27 y_{b}^{2}}{4}+20 g_{3}^{2}+\frac{45 g_{2}^{2}}{8}+\frac{5 g_{1}^{2}}{8}\right)+ \\
& \left.+y_{\tau}^{2}\left(-\frac{9 y_{\tau}^{2}}{4}+\frac{15 g_{2}^{2}}{8}+\frac{15 g_{1}^{2}}{8}\right)-\frac{145}{32} g_{2}^{4}+\frac{1671}{800} g_{1}^{4}+\frac{9 g_{2}^{2} g_{1}^{2}}{16}\right]+ \\
& +\frac{m^{2}}{(4 \pi)^{6}}\left[\lambda^{2}\left(1026 \lambda+\frac{297 y_{t}^{2}}{2}-192.822 g_{2}^{2}-38.564 g_{1}^{2}\right)+\lambda y_{t}^{2}\left(347.394 y_{t}^{2}+80.385 g_{3}^{2}+\right.\right. \\
& \left.-318.591 g_{2}^{2}-59.699 g_{1}^{2}\right)+\lambda\left(-64.5145 g_{2}^{4}-65.8056 g_{1}^{4}-37.8231 g_{2}^{2} g_{1}^{2}\right)+y_{t}^{4}\left(154.405 y_{t}^{2}+\right. \\
& \left.-209.24 g_{3}^{2}-3.82928 g_{2}^{2}-7.50769 g_{1}^{2}\right)+y_{t}^{2}\left(178.484 g_{3}^{4}-102.627 g_{2}^{4}-27.721 g_{1}^{4}+\right. \\
& \left.+7.572 g_{3}^{2} g_{2}^{2}+8.727 g_{3}^{2} g_{1}^{2}+11.470 g_{2}^{2} g_{1}^{2}\right)+g_{2}^{4}\left(-28.572 g_{3}^{2}+301.724 g_{2}^{2}+9.931 g_{1}^{2}\right)+ \\
& \left.+g_{1}^{4}\left(-4.191 g_{3}^{2}+9.778 g_{2}^{2}+8.378 g_{1}^{2}\right)\right]
\end{aligned}
$$

\section{B.4 Yukawa couplings}

RGE for the top Yukawa coupling:

$$
\begin{aligned}
\frac{d y_{t}^{2}}{d \ln \bar{\mu}^{2}}= & \frac{y_{t}^{2}}{(4 \pi)^{2}}\left[\frac{9 y_{t}^{2}}{2}+\frac{3 y_{b}^{2}}{2}+y_{\tau}^{2}-8 g_{3}^{2}-\frac{9 g_{2}^{2}}{4}-\frac{17 g_{1}^{2}}{20}\right]+ \\
& +\frac{y_{t}^{2}}{(4 \pi)^{4}}\left[y_{t}^{2}\left(-12 y_{t}^{2}-\frac{11 y_{b}^{2}}{4}-\frac{9 y_{\tau}^{2}}{4}-12 \lambda+36 g_{3}^{2}+\frac{225 g_{2}^{2}}{16}+\frac{393 g_{1}^{2}}{80}\right)+\right.
\end{aligned}
$$




$$
\begin{aligned}
& +y_{b}^{2}\left(-\frac{y_{b}^{2}}{4}+\frac{5 y_{\tau}^{2}}{4}+4 g_{3}^{2}+\frac{99 g_{2}^{2}}{16}+\frac{7 g_{1}^{2}}{80}\right)+y_{\tau}^{2}\left(-\frac{9 y_{\tau}^{2}}{4}+\frac{15}{8} g_{2}^{2}+\frac{15}{8} g_{1}^{2}\right)+ \\
& \left.+6 \lambda^{2}-108 g_{3}^{4}-\frac{23 g_{2}^{4}}{4}+\frac{1187 g_{1}^{4}}{600}+9 g_{3}^{2} g_{2}^{2}+\frac{19}{15} g_{3}^{2} g_{1}^{2}-\frac{9}{20} g_{2}^{2} g_{1}^{2}\right]+ \\
& +\frac{y_{t}^{2}}{(4 \pi)^{6}}\left[y_{t}^{4}\left(58.6028 y_{t}^{2}+198 \lambda-157 g_{3}^{2}-\frac{1593 g_{2}^{2}}{16}-\frac{2437 g_{1}^{2}}{80}\right)+\lambda y_{t}^{2}\left(\frac{15 \lambda}{4}+16 g_{3}^{2}+\right.\right. \\
& \left.-\frac{135 g_{2}^{2}}{2}-\frac{127 g_{1}^{2}}{10}\right)+y_{t}^{2}\left(363.764 g_{3}^{4}+16.990 g_{2}^{4}-24.422 g_{1}^{4}+48.370 g_{3}^{2} g_{2}^{2}+18.074 g_{3}^{2} g_{1}^{2}+\right. \\
& \left.+34.829 g_{2}^{2} g_{1}^{2}\right)+\lambda^{2}\left(-36 \lambda+45 g_{2}^{2}+9 g_{1}^{2}\right)+\lambda\left(-\frac{171 g_{2}^{4}}{16}-\frac{1089 g_{1}^{4}}{400}+\frac{117 g_{2}^{2} g_{1}^{2}}{40}\right)+ \\
& -619.35 g_{3}^{6}+169.829 g_{2}^{6}+16.099 g_{1}^{6}+73.654 g_{3}^{4} g_{2}^{2}-15.096 g_{3}^{4} g_{1}^{2}-21.072 g_{3}^{2} g_{2}^{4}+ \\
& \left.-22.319 g_{3}^{2} g_{1}^{4}-\frac{321}{20} g_{3}^{2} g_{2}^{2} g_{1}^{2}-4.743 g_{2}^{4} g_{1}^{2}-4.442 g_{2}^{2} g_{1}^{4}\right] .
\end{aligned}
$$

RGE for the bottom Yukawa coupling (up to two loops):

$$
\begin{aligned}
\frac{d y_{b}^{2}}{d \ln \bar{\mu}^{2}}= & \frac{y_{b}^{2}}{(4 \pi)^{2}}\left[\frac{3 y_{t}^{2}}{2}+\frac{9 y_{b}^{2}}{2}+y_{\tau}^{2}-8 g_{3}^{2}-\frac{9 g_{2}^{2}}{4}-\frac{g_{1}^{2}}{4}\right]+ \\
& +\frac{y_{b}^{2}}{(4 \pi)^{4}}\left[y_{t}^{2}\left(-\frac{y_{t}^{2}}{4}-\frac{11 y_{b}^{2}}{4}+\frac{5 y_{\tau}^{2}}{4}+4 g_{3}^{2}+\frac{99 g_{2}^{2}}{16}+\frac{91 g_{1}^{2}}{80}\right)+\right. \\
& +y_{b}^{2}\left(-12 y_{b}^{2}-\frac{9 y_{\tau}^{2}}{4}-12 \lambda+36 g_{3}^{2}+\frac{225 g_{2}^{2}}{16}+\frac{237 g_{1}^{2}}{80}\right)+y_{\tau}^{2}\left(-\frac{9 y_{\tau}^{2}}{4}+\frac{15}{8} g_{2}^{2}+\frac{15}{8} g_{1}^{2}\right)+ \\
& \left.+6 \lambda^{2}-108 g_{3}^{4}-\frac{23 g_{2}^{4}}{4}-\frac{127 g_{1}^{4}}{600}+9 g_{3}^{2} g_{2}^{2}+\frac{31}{15} g_{3}^{2} g_{1}^{2}-\frac{27}{20} g_{2}^{2} g_{1}^{2}\right] .
\end{aligned}
$$

RGE for the tau Yukawa coupling (up to two loops):

$$
\begin{aligned}
\frac{d y_{\tau}^{2}}{d \ln \bar{\mu}^{2}}= & \frac{y_{\tau}^{2}}{(4 \pi)^{2}}\left[3 y_{t}^{2}+3 y_{b}^{2}+\frac{5 y_{\tau}^{2}}{2}-\frac{9 g_{2}^{2}}{4}-\frac{9 g_{1}^{2}}{4}\right]+\frac{y_{\tau}^{2}}{(4 \pi)^{4}}\left[+6 \lambda^{2}-\frac{23 g_{2}^{4}}{4}+\frac{1371 g_{1}^{4}}{200}+\frac{27}{20} g_{2}^{2} g_{1}^{2}+\right. \\
& y_{t}^{2}\left(-\frac{27 y_{t}^{2}}{4}+\frac{3 y_{b}^{2}}{2}-\frac{27 y_{\tau}^{2}}{4}+20 g_{3}^{2}+\frac{45 g_{2}^{2}}{8}+\frac{17 g_{1}^{2}}{8}\right)+ \\
& \left.+y_{b}^{2}\left(-\frac{27 y_{b}^{2}}{4}-\frac{27 y_{\tau}^{2}}{4}+20 g_{3}^{2}+\frac{45 g_{2}^{2}}{8}+\frac{5 g_{1}^{2}}{8}\right)+y_{\tau}^{2}\left(-3 y_{\tau}^{2}-12 \lambda+\frac{165}{16} g_{2}^{2}+\frac{537}{80} g_{1}^{2}\right)\right] .
\end{aligned}
$$

\section{Effective potential at two loops}

The effective potential including one-loop and two-loop corrections in Landau gauge for $h \gg v$ is given by eq. (4.3), where $[8,23]$

$$
\lambda_{\mathrm{eff}}(h)=e^{4 \Gamma(h)}\left[\lambda(\bar{\mu}=h)+\lambda_{\mathrm{eff}}^{(1)}(\bar{\mu}=h)+\lambda_{\mathrm{eff}}^{(2)}(\bar{\mu}=h)\right] .
$$

All running couplings are evaluated at $\bar{\mu}=h$. Here, $\Gamma(h) \equiv \int_{M_{t}}^{h} \gamma(\bar{\mu}) d \ln \bar{\mu}$, with $\gamma$ the Higgs field anomalous dimension,

$$
\begin{aligned}
\gamma= & \frac{1}{(4 \pi)^{2}}\left[\frac{9}{4} g_{2}^{2}+\frac{9}{20} g_{1}^{2}-3 y_{t}^{2}-3 y_{b}^{2}-y_{\tau}^{2}\right]+ \\
& +\frac{1}{(4 \pi)^{4}}\left[y_{t}^{2}\left(-\frac{3 y_{b}^{2}}{2}-\frac{17 g_{1}^{2}}{8}-\frac{45 g_{2}^{2}}{8}-20 g_{3}^{2}+\frac{27 y_{t}^{2}}{4}\right)-y_{\tau}^{2}\left(\frac{15 g_{1}^{2}}{8}+\frac{15 g_{2}^{2}}{8}-\frac{9 y_{\tau}^{2}}{4}\right)+\right.
\end{aligned}
$$




$$
\begin{aligned}
& \left.+y_{b}^{2}\left(-\frac{5 g_{1}^{2}}{8}-\frac{45 g_{2}^{2}}{8}-20 g_{3}^{2}+\frac{27 y_{b}^{2}}{4}\right)-\frac{1293 g_{1}^{4}}{800}-\frac{27}{80} g_{2}^{2} g_{1}^{2}+\frac{271 g_{2}^{4}}{32}-6 \lambda^{2}\right]+ \\
& +\frac{1}{(4 \pi)^{6}}\left[-9 g_{1}^{2} \lambda^{2}-45 g_{2}^{2} \lambda^{2}+1.07 g_{1}^{4} \lambda+3.57 g_{2}^{2} g_{1}^{2} \lambda+8.92 g_{2}^{4} \lambda+\right. \\
& +14.99 g_{1}^{4} y_{t}^{2}+14.13 g_{1}^{2} y_{t}^{4}-13.21 g_{2}^{2} g_{1}^{2} y_{t}^{2}-8.73 g_{3}^{2} g_{1}^{2} y_{t}^{2}+40.11 g_{2}^{2} y_{t}^{4}+79.05 g_{3}^{2} y_{t}^{4}+ \\
& +23.40 g_{2}^{4} y_{t}^{2}-178.48 g_{3}^{4} y_{t}^{2}-7.57 g_{2}^{2} g_{3}^{2} y_{t}^{2}-5.26 g_{1}^{6}+1.93 g_{2}^{2} g_{1}^{4}+4.19 g_{3}^{2} g_{1}^{4}+ \\
& \left.+1.81 g_{2}^{4} g_{1}^{2}-158.51 g_{2}^{6}+28.57 g_{2}^{4} g_{3}^{2}+36 \lambda^{3}+\frac{135}{2} \lambda^{2} y_{t}^{2}-45.00 \lambda y_{t}^{4}-60.13 y_{t}^{6}\right] .
\end{aligned}
$$

The one-loop correction is

$$
\begin{aligned}
\lambda_{\text {eff }}^{(1)}= & \frac{1}{(4 \pi)^{2}}\left[\frac{3 g_{2}^{4}}{8}\left(\ln \frac{g_{2}^{2}}{4}-\frac{5}{6}+2 \Gamma\right)+\frac{3}{16}\left(g_{2}^{2}+g_{Y}^{2}\right)^{2}\left(\ln \frac{g_{2}^{2}+g_{Y}^{2}}{4}-\frac{5}{6}+2 \Gamma\right)+\right. \\
& \left.-3 y_{t}^{4}\left(\ln \frac{y_{t}^{2}}{2}-\frac{3}{2}+2 \Gamma\right)+3 \lambda^{2}(4 \ln \lambda-6+3 \ln 3+8 \Gamma)\right] .
\end{aligned}
$$

The two-loop correction is

$$
\begin{aligned}
\lambda_{\mathrm{eff}}^{(2)}= & \frac{1}{(4 \pi)^{4}}\left[8 g_{3}^{2} y_{t}^{4}\left(3 r_{t}^{2}-8 r_{t}+9\right)+\frac{1}{2} y_{t}^{6}\left(-6 r_{t} r_{W}-3 r_{t}^{2}+48 r_{t}-6 r_{t W}-69-\pi^{2}\right)+\right. \\
& +\frac{3 y_{t}^{2} g_{2}^{4}}{16}\left(8 r_{W}+4 r_{Z}-3 r_{t}^{2}-6 r_{t} r_{Z}-12 r_{t}+12 r_{t W}+15+2 \pi^{2}\right)+ \\
& +\frac{y_{t}^{2} g_{Y}^{4}}{48}\left(27 r_{t}^{2}-54 r_{t} r_{Z}-68 r_{t}-28 r_{Z}+189\right)+\frac{y_{t}^{2} g_{2}^{2} g_{Y}^{2}}{8}\left(9 r_{t}^{2}-18 r_{t} r_{Z}+4 r_{t}+44 r_{Z}-57\right)+ \\
& +\frac{g_{2}^{6}}{192}\left(36 r_{t} r_{Z}+54 r_{t}^{2}-414 r_{W} r_{Z}+69 r_{W}^{2}+1264 r_{W}+156 r_{Z}^{2}+632 r_{Z}-144 r_{t W}-2067+90 \pi^{2}\right)+ \\
& +\frac{g_{2}^{4} g_{Y}^{2}}{192}\left(12 r_{t} r_{Z}-6 r_{t}^{2}-6 r_{W}\left(53 r_{Z}+50\right)+213 r_{W}^{2}+4 r_{Z}\left(57 r_{Z}-91\right)+817+46 \pi^{2}\right)+ \\
& +\frac{g_{2}^{2} g_{Y}^{4}}{576}\left(132 r_{t} r_{Z}-66 r_{t}^{2}+306 r_{W} r_{Z}-153 r_{W}^{2}-36 r_{W}+924 r_{Z}^{2}-4080 r_{Z}+4359+218 \pi^{2}\right)+ \\
& +\frac{g_{Y}^{6}}{576}\left(6 r_{Z}\left(34 r_{t}+3 r_{W}-470\right)-102 r_{t}^{2}-9 r_{W}^{2}+708 r_{Z}^{2}+2883+206 \pi^{2}\right)+ \\
& +\frac{y_{t}^{4}}{6}\left(4 g_{Y}^{2}\left(3 r_{t}^{2}-8 r_{t}+9\right)-9 g_{2}^{2}\left(r_{t}-r_{W}+1\right)\right)+\frac{3}{4}\left(g_{2}^{6}-3 g_{2}^{4} y_{t}^{2}+4 y_{t}^{6}\right) L i_{2} \frac{g_{2}^{2}}{2 y_{t}^{2}}+ \\
& +\frac{y_{t}^{2}}{48} \xi\left(\frac{g_{2}^{2}+g_{Y}^{2}}{2 y_{t}^{2}}\right)\left(9 g_{2}^{4}-6 g_{2}^{2} g_{Y}^{2}+17 g_{Y}^{4}+2 y_{t}^{2}\left(7 g_{Y}^{2}-73 g_{2}^{2}+\frac{64 g_{2}^{4}}{g_{Y}^{2}+g_{2}^{2}}\right)\right)+ \\
& \left.+\frac{g_{2}^{2}}{64} \xi\left(\frac{g_{2}^{2}+g_{Y}^{2}}{g_{2}^{2}}\right)\left(18 g_{2}^{2} g_{Y}^{2}+g_{Y}^{4}-51 g_{2}^{4}-\frac{48 g_{2}^{6}}{g_{Y}^{2}+g_{2}^{2}}\right)\right] .
\end{aligned}
$$

Here we have given $\lambda_{\text {eff }}^{(2)}$ in the approximation $\lambda=0$, which is well justified around the instability region. The full expression of $\lambda_{\text {eff }}^{(2)}$ can be found in ref. [8]. Moreover, we have defined

$$
\xi(z) \equiv \sqrt{z^{2}-4 z}\left[2 \ln ^{2}\left(\frac{z-\sqrt{z^{2}-4 z}}{2 z}\right)-\ln ^{2} z-4 \operatorname{Li}_{2}\left(\frac{z-\sqrt{z^{2}-4 z}}{2 z}\right)+\frac{\pi^{2}}{3}\right],
$$

where $\mathrm{Li}_{2}$ is the dilogarithm function, and

$$
\begin{array}{ll}
r_{W}=\ln \frac{g_{2}^{2}}{4}+2 \Gamma, & r_{Z}=\ln \frac{g_{2}^{2}+g_{Y}^{2}}{4}+2 \Gamma, \quad r_{t}=\ln \frac{y_{t}^{2}}{2}+2 \Gamma, \\
r_{t W}=\left(r_{t}-r_{W}\right)\left[\ln \left(\frac{y_{t}^{2}}{2}-\frac{g_{2}^{2}}{4}\right)+2 \Gamma\right] . &
\end{array}
$$


Open Access. This article is distributed under the terms of the Creative Commons Attribution License which permits any use, distribution and reproduction in any medium, provided the original author(s) and source are credited.

\section{References}

[1] ATLAS collaboration, Observation of a new particle in the search for the Standard Model Higgs boson with the ATLAS detector at the LHC, Phys. Lett. B 716 (2012) 1 [arXiv: 1207.7214] [INSPIRE].

[2] CMS collaboration, Observation of a new boson at a mass of $125 \mathrm{GeV}$ with the CMS experiment at the LHC, Phys. Lett. B 716 (2012) 30 [arXiv:1207.7235] [INSPIRE].

[3] CMS collaboration, Updated measurements of the Higgs boson at $125 \mathrm{GeV}$ in the two photon decay channel, CMS-PAS-HIG-13-001, CERN, Geneva Switzerland (2013).

[4] ATLAS collaboration, Measurements of the properties of the Higgs-like boson in the two photon decay channel with the ATLAS detector using $25 \mathrm{fb}^{-1}$ of proton-proton collision data, ATLAS-CONF-2013-012, CERN, Geneva Switzerland (2013).

[5] CMS collaboration, Properties of the Higgs-like boson in the decay $H \rightarrow Z Z \rightarrow 4 \ell$ in $p p$ collisions at $\sqrt{s}=7$ and 8 TeV, CMS-PAS-HIG-13-002, CERN, Geneva Switzerland (2013).

[6] ATLAS collaboration, Measurements of the properties of the Higgs-like boson in the four lepton decay channel with the ATLAS detector using $25 \mathrm{fb}^{-1}$ of proton-proton collision data, ATLAS-CONF-2013-013, CERN, Geneva Switzerland (2013).

[7] P.P. Giardino, K. Kannike, I. Masina, M. Raidal and A. Strumia, The universal Higgs fit, arXiv: 1303.3570 [INSPIRE].

[8] G. Degrassi et al., Higgs mass and vacuum stability in the Standard Model at NNLO, JHEP 08 (2012) 098 [arXiv: 1205.6497] [INSPIRE].

[9] I. Krive and A.D. Linde, On the vacuum stability problem in gauge theories, Nucl. Phys. B 117 (1976) 265 [INSPIRE].

[10] N. Krasnikov, Restriction of the fermion mass in gauge theories of weak and electromagnetic interactions, Yad. Fiz. 28 (1978) 549 [INSPIRE].

[11] L. Maiani, G. Parisi and R. Petronzio, Bounds on the number and masses of quarks and leptons, Nucl. Phys. B 136 (1978) 115 [inSPIRE].

[12] H.D. Politzer and S. Wolfram, Bounds on particle masses in the Weinberg-Salam model, Phys. Lett. B 82 (1979) 242 [Erratum ibid. B 83 (1979) 421] [INSPIRE].

[13] P.Q. Hung, Vacuum instability and new constraints on fermion masses, Phys. Rev. Lett. 42 (1979) 873 [INSPIRE].

[14] N. Cabibbo, L. Maiani, G. Parisi and R. Petronzio, Bounds on the fermions and Higgs boson masses in grand unified theories, Nucl. Phys. B 158 (1979) 295 [INSPIRE].

[15] A.D. Linde, Vacuum instability, cosmology and constraints on particle masses in the Weinberg-Salam model, Phys. Lett. B 92 (1980) 119 [INSPIRE].

[16] M. Lindner, Implications of triviality for the Standard Model, Z. Phys. C 31 (1986) 295 [INSPIRE]. 
[17] M. Lindner, M. Sher and H.W. Zaglauer, Probing vacuum stability bounds at the Fermilab collider, Phys. Lett. B 228 (1989) 139 [InSPIRE].

[18] M. Sher, Electroweak Higgs potentials and vacuum stability, Phys. Rept. 179 (1989) 273 [INSPIRE].

[19] P.B. Arnold, Can the electroweak vacuum be unstable?, Phys. Rev. D 40 (1989) 613 [INSPIRE].

[20] P.B. Arnold and S. Vokos, Instability of hot electroweak theory: bounds on $m_{H}$ and $M_{t}$, Phys. Rev. D 44 (1991) 3620 [inSPIRE].

[21] M. Sher, Precise vacuum stability bound in the Standard Model, Phys. Lett. B 317 (1993) 159 [Addendum ibid. B 331 (1994) 448] [hep-ph/9307342] [INSPIRE].

[22] G. Altarelli and G. Isidori, Lower limit on the Higgs mass in the Standard Model: an update, Phys. Lett. B 337 (1994) 141 [InSPIRE].

[23] J.A. Casas, J.R. Espinosa and M. Quirós, Improved Higgs mass stability bound in the Standard Model and implications for supersymmetry, Phys. Lett. B 342 (1995) 171 [hep-ph/9409458] [INSPIRE].

[24] J.R. Espinosa and M. Quirós, Improved metastability bounds on the Standard Model Higgs mass, Phys. Lett. B 353 (1995) 257 [hep-ph/9504241] [inSPIRE].

[25] J. Casas, J. Espinosa and M. Quirós, Standard Model stability bounds for new physics within LHC reach, Phys. Lett. B 382 (1996) 374 [hep-ph/9603227] [INSPIRE].

[26] B. Schrempp and M. Wimmer, Top quark and Higgs boson masses: interplay between infrared and ultraviolet physics, Prog. Part. Nucl. Phys. 37 (1996) 1 [hep-ph/9606386] [INSPIRE].

[27] T. Hambye and K. Riesselmann, Matching conditions and Higgs mass upper bounds revisited, Phys. Rev. D 55 (1997) 7255 [hep-ph/9610272] [INSPIRE].

[28] G. Isidori, G. Ridolfi and A. Strumia, On the metastability of the Standard Model vacuum, Nucl. Phys. B 609 (2001) 387 [hep-ph/0104016] [inSPIRE].

[29] J. Espinosa, G. Giudice and A. Riotto, Cosmological implications of the Higgs mass measurement, JCAP 05 (2008) 002 [arXiv:0710.2484] [INSPIRE].

[30] J. Ellis, J. Espinosa, G. Giudice, A. Hoecker and A. Riotto, The probable fate of the Standard Model, Phys. Lett. B 679 (2009) 369 [arXiv:0906.0954] [InSPIRE].

[31] B. Feldstein, L.J. Hall and T. Watari, Landscape prediction for the Higgs boson and top quark masses, Phys. Rev. D 74 (2006) 095011 [hep-ph/0608121] [INSPIRE].

[32] M. Holthausen, K.S. Lim and M. Lindner, Planck scale boundary conditions and the Higgs mass, JHEP 02 (2012) 037 [arXiv: 1112.2415] [INSPIRE].

[33] J. Elias-Miro et al., Higgs mass implications on the stability of the electroweak vacuum, Phys. Lett. B 709 (2012) 222 [arXiv:1112.3022] [INSPIRE].

[34] C.-S. Chen and Y. Tang, Vacuum stability, neutrinos and dark matter, JHEP 04 (2012) 019 [arXiv: 1202.5717] [INSPIRE].

[35] O. Lebedev, On stability of the electroweak vacuum and the Higgs portal, Eur. Phys. J. C 72 (2012) 2058 [arXiv: 1203.0156] [INSPIRE]. 
[36] J. Elias-Miro, J.R. Espinosa, G.F. Giudice, H.M. Lee and A. Strumia, Stabilization of the electroweak vacuum by a scalar threshold effect, JHEP 06 (2012) 031 [arXiv:1203.0237] [INSPIRE].

[37] W. Rodejohann and H. Zhang, Impact of massive neutrinos on the Higgs self-coupling and electroweak vacuum stability, JHEP 06 (2012) 022 [arXiv:1203.3825] [INSPIRE].

[38] F. Bezrukov, M.Y. Kalmykov, B.A. Kniehl and M. Shaposhnikov, Higgs boson mass and new physics, JHEP 10 (2012) 140 [arXiv:1205.2893] [INSPIRE].

[39] A. Datta and S. Raychaudhuri, Vacuum stability constraints and LHC searches for a model with a universal extra dimension, Phys. Rev. D 87 (2013) 035018 [arXiv:1207.0476] [INSPIRE].

[40] S. Alekhin, A. Djouadi and S. Moch, The top quark and Higgs boson masses and the stability of the electroweak vacuum, Phys. Lett. B 716 (2012) 214 [arXiv:1207.0980] [INSPIRE].

[41] J. Chakrabortty, M. Das and S. Mohanty, Constraints on TeV scale Majorana neutrino phenomenology from the vacuum stability of the Higgs, Mod. Phys. Lett. A 28 (2013) 1350032 [arXiv: 1207.2027] [INSPIRE].

[42] L.A. Anchordoqui et al., Vacuum stability of Standard Model ${ }^{++}$, JHEP 02 (2013) 074 [arXiv:1208.2821] [INSPIRE].

[43] I. Masina, Higgs boson and top quark masses as tests of electroweak vacuum stability, Phys. Rev. D 87 (2013) 053001 [arXiv: 1209.0393] [INSPIRE].

[44] E.J. Chun, H.M. Lee and P. Sharma, Vacuum stability, perturbativity, EWPD and Higgs-to-diphoton rate in type II seesaw models, JHEP 11 (2012) 106 [arXiv:1209.1303] [INSPIRE].

[45] D.J. Chung, A.J. Long and L.-T. Wang, The $125 \mathrm{GeV}$ Higgs and electroweak phase transition model classes, Phys. Rev. D 87 (2013) 023509 [arXiv:1209.1819] [INSPIRE].

[46] W. Chao, M. Gonderinger and M.J. Ramsey-Musolf, Higgs vacuum stability, neutrino mass and dark matter, Phys. Rev. D 86 (2012) 113017 [arXiv:1210.0491] [INSPIRE].

[47] P. Bhupal Dev, D.K. Ghosh, N. Okada and I. Saha, $125 \mathrm{GeV}$ Higgs boson and the type-II seesaw model, JHEP 03 (2013) 150 [Erratum ibid. 05 (2013) 049] [arXiv:1301.3453] [INSPIRE].

[48] O. Lebedev and A. Westphal, Metastable electroweak vacuum: implications for inflation, Phys. Lett. B 719 (2013) 415 [arXiv:1210.6987] [InSPIRE].

[49] H.B. Nielsen, PREdicted the Higgs mass, arXiv:1212.5716 [INSPIRE].

[50] A. Kobakhidze and A. Spencer-Smith, Electroweak vacuum (in)stability in an inflationary universe, Phys. Lett. B 722 (2013) 130 [arXiv:1301.2846] [INSPIRE].

[51] Y. Tang, Vacuum stability in the Standard Model, Mod. Phys. Lett. A 28 (2013) 1330002 [arXiv:1301.5812] [INSPIRE].

[52] F. Klinkhamer, Standard Model Higgs field and energy scale of gravity, JETP Lett. 97 (2013) 297 [arXiv: 1302.1496] [inSPIRE].

[53] X.-G. He, H. Phoon, Y. Tang and G. Valencia, Unitarity and vacuum stability constraints on the couplings of color octet scalars, JHEP 05 (2013) 026 [arXiv:1303.4848] [INSPIRE].

[54] E.J. Chun, S. Jung and H.M. Lee, Radiative generation of the Higgs potential, Phys. Lett. B 725 (2013) 158 [arXiv:1304.5815] [inSPIRE]. 
[55] F. Jegerlehner, The Standard Model as a low-energy effective theory: what is triggering the Higgs mechanism?, arXiv:1304.7813 [INSPIRE].

[56] O. Antipin, M. Gillioz, J. Krog, E. Mølgaard and F. Sannino, Standard Model vacuum stability and Weyl consistency conditions, JHEP 08 (2013) 034 [arXiv:1306.3234] [INSPIRE].

[57] V. Branchina and E. Messina, Stability, Higgs boson mass and new physics, arXiv: 1307.5193 [INSPIRE].

[58] D.J. Gross and F. Wilczek, Ultraviolet behavior of non-Abelian gauge theories, Phys. Rev. Lett. 30 (1973) 1343 [INSPIRE].

[59] H.D. Politzer, Reliable perturbative results for strong interactions?, Phys. Rev. Lett. 30 (1973) 1346 [INSPIRE].

[60] W.E. Caswell, Asymptotic behavior of non-Abelian gauge theories to two loop order, Phys. Rev. Lett. 33 (1974) 244 [INSPIRE].

[61] D. Jones, Two loop diagrams in Yang-Mills theory, Nucl. Phys. B 75 (1974) 531 [inSPIRE].

[62] O. Tarasov, A. Vladimirov and A.Y. Zharkov, The Gell-Mann-Low function of QCD in the three loop approximation, Phys. Lett. B 93 (1980) 429 [INSPIRE].

[63] S. Larin and J. Vermaseren, The three loop QCD $\beta$-function and anomalous dimensions, Phys. Lett. B 303 (1993) 334 [hep-ph/9302208] [INSPIRE].

[64] T. van Ritbergen, J. Vermaseren and S. Larin, The four loop $\beta$-function in quantum chromodynamics, Phys. Lett. B 400 (1997) 379 [hep-ph/9701390] [INSPIRE].

[65] M. Czakon, The four-loop QCD $\beta$-function and anomalous dimensions, Nucl. Phys. B 710 (2005) 485 [hep-ph/0411261] [INSPIRE].

[66] D. Jones, The two loop $\beta$-function for a $G_{1} \times G_{2}$ gauge theory, Phys. Rev. D 25 (1982) 581 [INSPIRE].

[67] M. Steinhauser, Higgs decay into gluons up to $O\left(\alpha_{s}^{3} G_{F} m_{t}^{2}\right)$, Phys. Rev. D 59 (1999) 054005 [hep-ph/9809507] [INSPIRE].

[68] M.E. Machacek and M.T. Vaughn, Two loop renormalization group equations in a general quantum field theory. 1. Wave function renormalization, Nucl. Phys. B 222 (1983) 83 [INSPIRE].

[69] L.N. Mihaila, J. Salomon and M. Steinhauser, Gauge coupling $\beta$-functions in the Standard Model to three loops, Phys. Rev. Lett. 108 (2012) 151602 [arXiv:1201.5868] [INSPIRE].

[70] L.N. Mihaila, J. Salomon and M. Steinhauser, Renormalization constants and $\beta$-functions for the gauge couplings of the Standard Model to three-loop order, Phys. Rev. D 86 (2012) 096008 [arXiv: 1208.3357] [INSPIRE].

[71] T. Cheng, E. Eichten and L.-F. Li, Higgs phenomena in asymptotically free gauge theories, Phys. Rev. D 9 (1974) 2259 [InSPIRE].

[72] M. Fischler and J. Oliensis, Two loop corrections to the evolution of the Higgs-Yukawa coupling constant, Phys. Lett. B 119 (1982) 385 [INSPIRE].

[73] K. Chetyrkin and M. Zoller, Three-loop $\beta$-functions for top-Yukawa and the Higgs self-interaction in the Standard Model, JHEP 06 (2012) 033 [arXiv:1205.2892] [INSPIRE]. 
[74] A. Bednyakov, A. Pikelner and V. Velizhanin, Yukawa coupling $\beta$-functions in the Standard Model at three loops, Phys. Lett. B 722 (2013) 336 [arXiv:1212.6829] [InSPIRE].

[75] M.E. Machacek and M.T. Vaughn, Two loop renormalization group equations in a general quantum field theory. 2. Yukawa couplings, Nucl. Phys. B 236 (1984) 221 [inSPIRE].

[76] M.E. Machacek and M.T. Vaughn, Two loop renormalization group equations in a general quantum field theory. 3. Scalar quartic couplings, Nucl. Phys. B 249 (1985) 70 [inSPIRE].

[77] M.-X. Luo and Y. Xiao, Two loop renormalization group equations in the Standard Model, Phys. Rev. Lett. 90 (2003) 011601 [hep-ph/0207271] [INSPIRE].

[78] K. Chetyrkin and M. Zoller, $\beta$-function for the Higgs self-interaction in the Standard Model at three-loop level, JHEP 04 (2013) 091 [Erratum ibid. 09 (2013) 155] [arXiv:1303.2890] [INSPIRE].

[79] A. Bednyakov, A. Pikelner and V. Velizhanin, Higgs self-coupling $\beta$-function in the Standard Model at three loops, Nucl. Phys. B 875 (2013) 552 [arXiv:1303.4364] [INSPIRE].

[80] A. Sirlin, Radiative corrections in the $\mathrm{SU}(2)_{L} \times \mathrm{U}(1)$ theory: a simple renormalization framework, Phys. Rev. D 22 (1980) 971 [INSPIRE].

[81] W. Marciano and A. Sirlin, Radiative corrections to neutrino induced neutral current phenomena in the $\mathrm{SU}(2)_{L} \times \mathrm{U}(1)$ theory, Phys. Rev. D 22 (1980) 2695 [Erratum ibid. D 31 (1985) 213] [INSPIRE].

[82] R. Tarrach, The pole mass in perturbative QCD, Nucl. Phys. B 183 (1981) 384 [InSPIRE].

[83] K. Chetyrkin and M. Steinhauser, Short distance mass of a heavy quark at order $\alpha_{s}^{3}$, Phys. Rev. Lett. 83 (1999) 4001 [hep-ph/9907509] [INSPIRE].

[84] K. Chetyrkin and M. Steinhauser, The relation between the MS-bar and the on-shell quark mass at order $\alpha_{s}^{3}$, Nucl. Phys. B 573 (2000) 617 [hep-ph/9911434] [INSPIRE].

[85] K. Melnikov and T.v. Ritbergen, The three loop relation between the MS-bar and the pole quark masses, Phys. Lett. B 482 (2000) 99 [hep-ph/9912391] [INSPIRE].

[86] R. Hempfling and B.A. Kniehl, On the relation between the fermion pole mass and MS Yukawa coupling in the Standard Model, Phys. Rev. D 51 (1995) 1386 [hep-ph/9408313] [INSPIRE].

[87] A. Sirlin and R. Zucchini, Dependence of the quartic coupling $H_{m}$ on $M_{H}$ and the possible onset of new physics in the Higgs sector of the Standard Model, Nucl. Phys. B 266 (1986) 389 [INSPIRE].

[88] W.E. Caswell and F. Wilczek, On the gauge dependence of renormalization group parameters, Phys. Lett. B 49 (1974) 291 [INSPIRE].

[89] T. Muta, Foundations of quantum chromodynamics, World Scientific, Singapore (2010), pg. 192.

[90] D. Atkinson and M. Fry, Should one truncate the electron selfenergy?, Nucl. Phys. B 156 (1979) 301 [inSPIRE].

[91] J. Breckenridge, M. Lavelle and T.G. Steele, The Nielsen identities for the two point functions of QED and QCD, Z. Phys. C 65 (1995) 155 [hep-th/9407028] [INSPIRE].

[92] A.S. Kronfeld, The perturbative pole mass in QCD, Phys. Rev. D 58 (1998) 051501 [hep-ph/9805215] [INSPIRE]. 
[93] Tevatron Electroweak Working Group, CDF and D0 collaborations, 2012 update of the combination of CDF and D0 results for the mass of the $W$ boson, FERMILAB-TM-2532-E, Fermilab, Batavia U.S.A. (2012) [arXiv:1204.0042] [INSPIRE].

[94] ALEPH, DELPHI, L3, OPAL and LEP Electroweak Working Group collaborations, A combination of preliminary electroweak measurements and constraints on the Standard Model, 2006, CERN-PH-EP-2006-042, CERN, Geneva Switzerland (2006) [hep-ex/0612034] [INSPIRE].

[95] 2012 Particle Data Group average, http://pdg.lbl.gov/.

[96] Tevatron Electroweak Working Group, CDF and D0 collaborations, Combination of CDF and D0 results on the mass of the top quark using up to $5.8 \mathrm{fb}^{-1}$ of data, arXiv:1107.5255 [INSPIRE].

[97] CMS collaboration, CMS top physics website, https://twiki.cern.ch/twiki/bin/view/CMSPublic/PhysicsResultsTOP.

[98] CMS collaboration, talks at the Moriond 2013 conference, La Thuile Italy March 9-16 2013.

[99] ATLAS collaboration, ATLAS top physics website, https://twiki.cern.ch/twiki/bin/view/AtlasPublic/TopPublicResults.

[100] MuLan collaboration, V. Tishchenko et al., Detailed report of the MuLan measurement of the positive muon lifetime and determination of the Fermi constant, Phys. Rev. D 87 (2013) 052003 [arXiv:1211.0960] [INSPIRE].

[101] S. Bethke, World summary of $\alpha_{s}$ (2012), Nucl. Phys. Proc. Suppl. 234 (2013) 229 [arXiv:1210.0325] [INSPIRE].

[102] T. Kinoshita and A. Sirlin, Radiative corrections to Fermi interactions, Phys. Rev. 113 (1959) 1652 [INSPIRE].

[103] T. van Ritbergen and R.G. Stuart, On the precise determination of the Fermi coupling constant from the muon lifetime, Nucl. Phys. B 564 (2000) 343 [hep-ph/9904240] [INSPIRE].

[104] T. Hahn, Generating Feynman diagrams and amplitudes with FeynArts 3, Comput. Phys. Commun. 140 (2001) 418 [hep-ph/0012260] [INSPIRE].

[105] R. Mertig and R. Scharf, TARCER: a Mathematica program for the reduction of two loop propagator integrals, Comput. Phys. Commun. 111 (1998) 265 [hep-ph/9801383] [INSPIRE].

[106] O. Tarasov, Generalized recurrence relations for two loop propagator integrals with arbitrary masses, Nucl. Phys. B 502 (1997) 455 [hep-ph/9703319] [INSPIRE].

[107] R. Mertig, M. Böhm and A. Denner, FEYN CALC: computer algebraic calculation of Feynman amplitudes, Comput. Phys. Commun. 64 (1991) 345 [INSPIRE].

[108] M. Awramik, M. Czakon, A. Onishchenko and O. Veretin, Bosonic corrections to $\Delta r$ at the two loop level, Phys. Rev. D 68 (2003) 053004 [hep-ph/0209084] [InSPIRE].

[109] S.P. Martin, Evaluation of two loop selfenergy basis integrals using differential equations, Phys. Rev. D 68 (2003) 075002 [hep-ph/0307101] [inSPIRE].

[110] S.P. Martin and D.G. Robertson, TSIL: a program for the calculation of two-loop self-energy integrals, Comput. Phys. Commun. 174 (2006) 133 [hep-ph/0501132] [InSPIRE].

[111] B.A. Kniehl and A. Sirlin, Pole mass, width and propagators of unstable Fermions, Phys. Rev. D 77 (2008) 116012 [arXiv:0801.0669] [InSPIRE]. 
[112] F. Jegerlehner, M.Y. Kalmykov and B.A. Kniehl, On the difference between the pole and the MS-bar masses of the top quark at the electroweak scale, Phys. Lett. B 722 (2013) 123 [arXiv:1212.4319] [INSPIRE].

[113] S.P. Martin, Three-loop Standard Model effective potential at leading order in strong and top Yukawa couplings, arXiv:1310.7553 [INSPIRE].

[114] Particle Data Group collaboration, K. Nakamura et al., Review of particle physics, J. Phys. G 37 (2010) 075021 [inSPIRE].

[115] LEP Electroweak Working Group webpage, http://lepewwg.web.cern.ch/.

[116] C. Froggatt and H.B. Nielsen, Standard Model criticality prediction: top mass $173 \pm 5 \mathrm{GeV}$ and Higgs mass $135 \pm 9 \mathrm{GeV}$, Phys. Lett. B 368 (1996) 96 [hep-ph/9511371] [INSPIRE].

[117] C. Froggatt, H.B. Nielsen and Y. Takanishi, Standard Model Higgs boson mass from borderline metastability of the vacuum, Phys. Rev. D 64 (2001) 113014 [hep-ph/0104161] [INSPIRE].

[118] C. Burgess, V. Di Clemente and J. Espinosa, Effective operators and vacuum instability as heralds of new physics, JHEP 01 (2002) 041 [hep-ph/0201160] [INSPIRE].

[119] G. Isidori, V.S. Rychkov, A. Strumia and N. Tetradis, Gravitational corrections to Standard Model vacuum decay, Phys. Rev. D 77 (2008) 025034 [arXiv: 0712.0242] [INSPIRE].

[120] F. Bezrukov and M. Shaposhnikov, Standard Model Higgs boson mass from inflation: two loop analysis, JHEP 07 (2009) 089 [arXiv:0904.1537] [INSPIRE].

[121] M. Shaposhnikov and C. Wetterich, Asymptotic safety of gravity and the Higgs boson mass, Phys. Lett. B 683 (2010) 196 [arXiv:0912.0208] [INSPIRE].

[122] C. Ford, I. Jack and D. Jones, The Standard Model effective potential at two loops, Nucl. Phys. B 387 (1992) 373 [Erratum ibid. B 504 (1997) 551] [hep-ph/0111190] [INSPIRE].

[123] L.J. Hall and Y. Nomura, A finely-predicted Higgs boson mass from a finely-tuned weak scale, JHEP 03 (2010) 076 [arXiv:0910.2235] [INSPIRE].

[124] G.F. Giudice and A. Strumia, Probing high-scale and split supersymmetry with Higgs mass measurements, Nucl. Phys. B 858 (2012) 63 [arXiv:1108.6077] [INSPIRE].

[125] M. Cabrera, J. Casas and A. Delgado, Upper bounds on superpartner masses from upper bounds on the Higgs boson mass, Phys. Rev. Lett. 108 (2012) 021802 [arXiv:1108.3867] [INSPIRE].

[126] A. Arbey, M. Battaglia, A. Djouadi, F. Mahmoudi and J. Quevillon, Implications of a 125 GeV Higgs for supersymmetric models, Phys. Lett. B 708 (2012) 162 [arXiv: 1112.3028] [INSPIRE].

[127] L.E. Ibá nez and I. Valenzuela, The Higgs mass as a signature of heavy SUSY, JHEP 05 (2013) 064 [arXiv:1301.5167] [InSPIRE].

[128] A. Hebecker, A.K. Knochel and T. Weigand, The Higgs mass from a string-theoretic perspective, Nucl. Phys. B 874 (2013) 1 [arXiv:1304.2767] [INSPIRE].

[129] P.J. Fox, A.E. Nelson and N. Weiner, Dirac gaugino masses and supersoft supersymmetry breaking, JHEP 08 (2002) 035 [hep-ph/0206096] [INSPIRE].

[130] K. Benakli, M.D. Goodsell and F. Staub, Dirac gauginos and the $125 \mathrm{GeV}$ Higgs, JHEP 06 (2013) 073 [arXiv: 1211.0552] [INSPIRE]. 
[131] A. Hebecker, A.K. Knochel and T. Weigand, A shift symmetry in the Higgs sector: experimental hints and stringy realizations, JHEP 06 (2012) 093 [arXiv:1204.2551] [INSPIRE].

[132] M. Redi and A. Strumia, Axion-Higgs unification, JHEP 11 (2012) 103 [arXiv:1208.6013] [INSPIRE].

[133] G. Giudice and R. Rattazzi, Living dangerously with low-energy supersymmetry, Nucl. Phys. B 757 (2006) 19 [hep-ph/0606105] [INSPIRE].

[134] P. Bak, C. Tang and K. Wiesenfeld, Self-organized criticality: an explanation of $1 / f$ noise, Phys. Rev. Lett. 59 (1987) 381 [INSPIRE].

[135] S. Weinberg, Anthropic bound on the cosmological constant, Phys. Rev. Lett. 59 (1987) 2607 [INSPIRE].

[136] J.F. Donoghue, K. Dutta and A. Ross, Quark and lepton masses and mixing in the landscape, Phys. Rev. D 73 (2006) 113002 [hep-ph/0511219] [inSPIRE].

[137] L.J. Hall, M.P. Salem and T. Watari, Quark and lepton masses from Gaussian landscapes, Phys. Rev. Lett. 100 (2008) 141801 [arXiv:0707.3444] [InSPIRE].

[138] G.W. Gibbons, S. Gielen, C. Pope and N. Turok, Measures on mixing angles, Phys. Rev. D 79 (2009) 013009 [arXiv: 0810.4813] [INSPIRE].

[139] G.F. Giudice, G. Perez and Y. Soreq, Flavor beyond the standard universe, arXiv: 1207.4861 [INSPIRE].

[140] N. Arkani-Hamed, S. Dimopoulos and S. Kachru, Predictive landscapes and new physics at a $T e V$, hep-th/0501082 [INSPIRE].

[141] V. Agrawal, S.M. Barr, J.F. Donoghue and D. Seckel, The anthropic principle and the mass scale of the Standard Model, Phys. Rev. D 57 (1998) 5480 [hep-ph/9707380] [INSPIRE].

[142] I.Y. Kobzarev, L. Okun and M. Voloshin, Bubbles in metastable vacuum, Sov. J. Nucl. Phys. 20 (1975) 644 [Yad. Fiz. 20 (1974) 1229] [INSPIRE].

[143] S.R. Coleman, The fate of the false vacuum. 1. Semiclassical theory, Phys. Rev. D 15 (1977) 2929 [Erratum ibid. D 16 (1977) 1248] [INSPIRE].

[144] C.G. Callan Jr. and S.R. Coleman, The fate of the false vacuum. 2. First quantum corrections, Phys. Rev. D 16 (1977) 1762 [InSPIRE].

[145] S.R. Coleman and F. De Luccia, Gravitational effects on and of vacuum decay, Phys. Rev. D 21 (1980) 3305 [INSPIRE]. 